\title{
Crystal Engineering of Angular-Shaped Heteroarenes Based on Cyclopenta $[b]$ thiopyran for Controlling the Charge Carrier Mobility
}

Yanjun Qiao, ${ }^{\dagger}, \#$ Longfei Yang, ${ }^{\dagger, \#}$ Jiangyu Zhu, ${ }^{\dagger}$ Chuan Yan, ${ }^{\ddagger}$ Dongdong Chang, ${ }^{\dagger}$ Ning Zhang, ${ }^{\dagger}$ Gang Zhou, ${ }^{*, *}$ Yan Zhao, ${ }^{*, \dagger}$ Xuefeng Lu, ${ }^{*, \dagger}$ and Yunqi Liu*,†

†Department of Materials Science, Fudan University, Shanghai 200433, China

Lab of Advanced Materials, State Key Laboratory of Molecular Engineering of Polymers, Fudan University, Shanghai 200433, China

\section{Table of Contents}

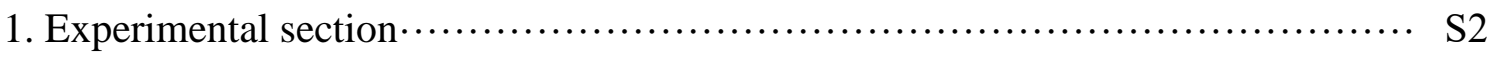

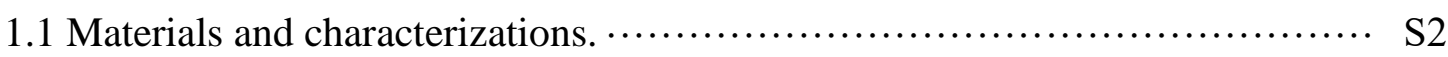

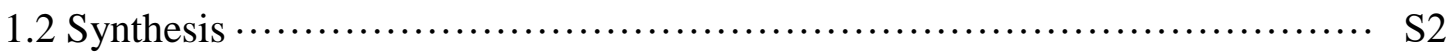

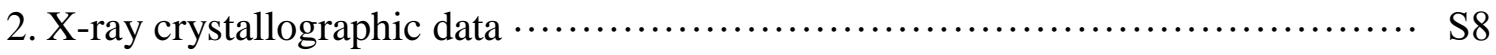

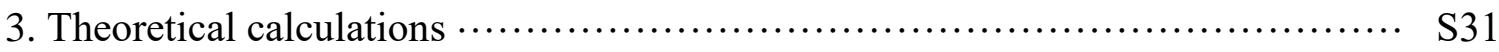

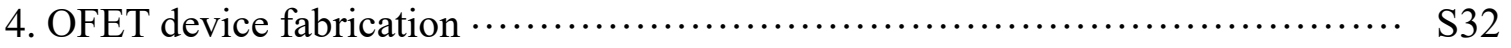

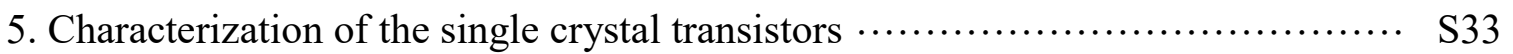

6. ${ }^{1} \mathrm{H}$ and ${ }^{13} \mathrm{C}$ NMR spectra of new compounds .................................. S37

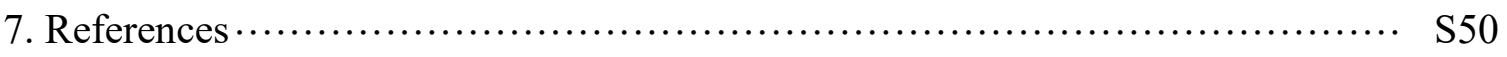




\section{Experimental section.}

\subsection{Materials and characterizations.}

All chemicals and reagents were purchased from commercial sources and used as received unless otherwise specified. 2,6-Dibromonaphthalene-1,5-diyl bis(trifluoromethanesulfonate), 1,5-di(1-hexynyl)-2,6-dibromonaphthalene, 1,5-di(1-octynyl)-2,6-dibromonaphthalene, 1,5di(1-decynyl)-2,6-dibromonaphthalene, and 1,5-di(1-dodecynyl)-2,6-dibromonaphthalene was synthesized following a previously reported method. ${ }^{\mathrm{S} 1}$ 4-Chlorothiophene-2-boronic acid pinacol ester was synthesized by following reported procedures. ${ }^{\mathrm{S} 2}$ All reactions and manipulations were carried out with the use of standard inert atmosphere and Schlenk techniques.

${ }^{1} \mathrm{H}$ NMR (400 MHz) and ${ }^{13} \mathrm{C}$ NMR (100 MHz) spectra were measured on a Varian Mercury Plus-400 spectrometer. The splitting patterns are designated as follows: s (singlet); $\mathrm{d}$ (doublet); t (triplet); m (multiplet). Ultraviolet-visible-near infrared (UV-vis-NIR) absorption spectra were acquired from PerkinElmer Lambda 750 spectrophotometer. Cyclic and differential pulse voltammetry (CV) measurements were performed using a CHI660E electrochemical workstation in a three-electrode electrochemical cell. A carbon glass coated electrode was used as the working electrode and an $\mathrm{Ag} / \mathrm{Ag}^{+}$electrode as the reference electrode, while $0.1 \mathrm{M}$ tetrabutylammonium hexafluorophosphate $\left(\mathrm{TBAPF}_{6}\right)$ in dichloromethane was the electrolyte, and ferrocene was used as internal standard to estimate frontier orbital energy levels of S-fused heteroarenes. The polarized light images were obtained from a polarized light microscope (Nikon LV150). The single crystal transistors were fabricated and measured on a Micromanipulator station (Everbeing) coupled with an optical microscope (PSM-1000). The current-voltage $(I-V)$ characteristics of the device were measured on the Micromanipulator station using a Keithley 4200 source meter under ambient conditions.

\subsection{Synthesis.}

\section{Synthesis of 1,5-di(1-hexynyl)-2,6-di(2-thienyl)naphthalene (3a).}

To a degassed solution of $2 \mathbf{a}(0.50 \mathrm{~g}, 1.12 \mathrm{mmol})$ in DMF $(10 \mathrm{~mL})$ was added $\mathrm{Pd}\left(\mathrm{PPh}_{3}\right)_{2} \mathrm{Cl}_{2}$ $(0.078 \mathrm{~g}, 0.11 \mathrm{mmol})$ and 2-(tri- $n$-butylstannyl)thiophene $(1.04 \mathrm{~g}, 2.8 \mathrm{mmol})$. Then the mixture was stirred at $90^{\circ} \mathrm{C}$ overnight. After removing the solvents in vacuo, the mixture was seperated and purified by column chromatography (eluted with PE/DCM $=10 / 1$ ) to give a brownish-yellow solid (0.45 g, yield: 89\%). ${ }^{1} \mathrm{H}$ NMR (400 MHz, $\left.\mathrm{CDCl}_{3}\right) \delta(\mathrm{ppm}): 8.41$ (d, $\left.J=8.8 \mathrm{~Hz}, 2 \mathrm{H}\right), 7.81$ (m, 4H), 7.43 (d, $J=3.6 \mathrm{~Hz}, 2 \mathrm{H}), 7.18$ (t, $J=3.2 \mathrm{~Hz}, 2 \mathrm{H}), 2.66$ (m, 4H), $1.77(\mathrm{~m}, 4 \mathrm{H}), 1.39$ 
(m, 4H), $1.02(\mathrm{~m}, 6 \mathrm{H}) .{ }^{13} \mathrm{C} \mathrm{NMR}\left(100 \mathrm{MHz}, \mathrm{CDCl}_{3}\right) \delta(\mathrm{ppm}): 143.0,134.3,133.4,128.0,127.5$, 127.3, 127.1, 126.4, 118.2, 102.3, 78.4, 30.8, 22.5, 20.2, 14.0. MALDI-TOF MS (m/z): $\left[\mathrm{M}^{+}\right]$ calcd for $\mathrm{C}_{30} \mathrm{H}_{28} \mathrm{~S}_{2}$, 452.1632; found 452.1068 .

\section{Synthesis of 1,5-di(1-octynyl)-2,6-di(2-selenophenyl)naphthalene (3b).}

To a degassed solution of $\mathbf{2 b}(0.34 \mathrm{~g}, 0.68 \mathrm{mmol})$ in DMF $(5 \mathrm{~mL})$ was added $\mathrm{Pd}\left(\mathrm{PPh}_{3}\right)_{2} \mathrm{Cl}_{2}$ (0.048 g, $0.068 \mathrm{mmol})$ and 2-(tri-n-butylstannyl)thiophene $(0.58 \mathrm{~g}, 1.6 \mathrm{mmol})$. Then the mixture was stirred at $90{ }^{\circ} \mathrm{C}$ overnight. After removing the solvents in vacuo, the mixture was seperated and purified by column chromatography (eluted with $\mathrm{PE} / \mathrm{DCM}=10 / 1$ ) to give a pale yellow solid (0.31 g, yield: 90\%). ${ }^{1} \mathrm{H}$ NMR (400 MHz, $\left.\mathrm{CDCl}_{3}\right) \delta(\mathrm{ppm}): 8.41(\mathrm{~d}, J=8.8 \mathrm{~Hz}$, 2H), $7.79(\mathrm{~m}, 4 \mathrm{H}), 7.42(\mathrm{~d}, J=4.8 \mathrm{~Hz}, 2 \mathrm{H}), 7.16(\mathrm{t}, J=4.8 \mathrm{~Hz}, 2 \mathrm{H}), 2.64(\mathrm{t}, J=6.8 \mathrm{~Hz}, 4 \mathrm{H})$, $1.77(\mathrm{~m}, 4 \mathrm{H}), 1.54(\mathrm{~m}, 4 \mathrm{H}), 1.37(\mathrm{~m}, 8 \mathrm{H}), 0.94(\mathrm{~m}, 6 \mathrm{H}) .{ }^{13} \mathrm{C} \mathrm{NMR}\left(100 \mathrm{MHz}, \mathrm{CDCl}_{3}\right) \delta(\mathrm{ppm})$ : 143.0, 134.3, 133.4, 128.0, 127.4, 127.3, 127.1, 126.3, 118.2, 102.3, 78.4, 31.7, 29.1, 28.7, 22.9, 20.5, 14.4. MALDI-TOF MS (m/z): $\left[\mathrm{M}^{+}\right]$calcd for $\mathrm{C}_{34} \mathrm{H}_{36} \mathrm{~S}_{2}, 508.2258$; found 508.1458.

\section{Synthesis of 1,5-di(1-decynyl)-2,6-di(2-thienyl)naphthalene (3c).}

To a degassed solution of $2 \mathbf{c}(0.49 \mathrm{~g}, 0.88 \mathrm{mmol})$ in dry DMF $(10 \mathrm{~mL})$ was added $\mathrm{Pd}\left(\mathrm{PPh}_{3}\right)_{2} \mathrm{Cl}_{2}$ $(0.062 \mathrm{~g}, 0.088 \mathrm{mmol})$ and then heated at $90{ }^{\circ} \mathrm{C}$. Tributyl(2-thienyl)stannane $(0.82 \mathrm{~g}, 2.2 \mathrm{mmol})$ was added and the mixture was stirred at $90{ }^{\circ} \mathrm{C}$ overnight. After removing the solvents in vacuum, the mixture was separated and purified by column chromatography eluted with $\mathrm{PE} / \mathrm{DCM}=10 / 1$ to get a brownish-yellow solid $(0.46 \mathrm{~g}$, yield: $92 \%) .{ }^{1} \mathrm{H}$ NMR (400 MHz, $\left.\mathrm{CDCl}_{3}\right) \delta(\mathrm{ppm}): 8.39(\mathrm{~d}, J=9.0 \mathrm{~Hz}, 2 \mathrm{H}), 7.78(\mathrm{~d}, J=9.0 \mathrm{~Hz}, 2 \mathrm{H}), 7.74(\mathrm{~d}, J=3.3 \mathrm{~Hz}, 2 \mathrm{H})$, $7.41(\mathrm{~d}, J=4.9 \mathrm{~Hz}, 2 \mathrm{H}), 7.15(\mathrm{t}, J=3.8 \mathrm{~Hz}, 2 \mathrm{H}), 2.62(\mathrm{t}, J=6.9 \mathrm{~Hz}, 4 \mathrm{H}), 1.71(\mathrm{~m}, 4 \mathrm{H}), 1.51$ $(\mathrm{m}, 4 \mathrm{H}), 1.29(\mathrm{~m}, 16 \mathrm{H}), 0.88(\mathrm{t}, J=6.6 \mathrm{~Hz}, 6 \mathrm{H}) .{ }^{13} \mathrm{C} \mathrm{NMR}\left(100 \mathrm{MHz}, \mathrm{CDCl}_{3}\right) \delta(\mathrm{ppm}): 142.9$, 134.3, 133.4, 127.9, 127.4, 127.2, 127.1, 126.3, 118.1, 102.3, 78.4, 32.1, 29.5, 29.4, 29.4, 28.6, 22.9, 20.4, 14.3. MALDI-TOF MS (m/z): $[\mathrm{M}+\mathrm{H}]^{+}$calcd for $\mathrm{C}_{38} \mathrm{H}_{45} \mathrm{~S}_{2}, 565.2963$; found. 565.2935 .

\section{Synthesis of 1,5-di(1-dodecynyl)-2,6-di(2-thienyl)naphthalene (3d).}

To a degassed solution of $\mathbf{2 d}(0.40 \mathrm{~g}, 0.65 \mathrm{mmol})$ in dry DMF $(5 \mathrm{~mL})$ was added $\mathrm{Pd}\left(\mathrm{PPh}_{3}\right)_{2} \mathrm{Cl}_{2}$ $(0.046 \mathrm{~g}, 0.065 \mathrm{mmol})$ and then heated at $90{ }^{\circ} \mathrm{C}$. Tributyl(2-thienyl)stannane $(0.61 \mathrm{~g}, 1.63$ mmol) was added and the mixture was stirred at $90{ }^{\circ} \mathrm{C}$ overnight. After removing the solvents in vacuum, the mixture was separated and purified by column chromatography eluted with $\mathrm{PE} / \mathrm{DCM}=10 / 1$ to get a brownish-yellow solid $(0.35 \mathrm{~g}$, yield: $88 \%) .{ }^{1} \mathrm{H}$ NMR $(400 \mathrm{MHz}$, $\left.\mathrm{CDCl}_{3}\right) \delta(\mathrm{ppm}): 8.41(\mathrm{~d}, J=8.8 \mathrm{~Hz}, 2 \mathrm{H}), 7.80(\mathrm{~m}, 4 \mathrm{H}), 7.42(\mathrm{~d}, J=5.2 \mathrm{~Hz}, 2 \mathrm{H}), 7.17(\mathrm{t}, J=$ 
4.0 Hz, 2H), 2.64 (t, J=7.2 Hz, 4H), $1.77(\mathrm{~m}, 4 \mathrm{H}), 1.54(\mathrm{~m}, 4 \mathrm{H}), 1.39$ (m, 24H), $0.95(\mathrm{~m}, 6 \mathrm{H})$. ${ }^{13} \mathrm{C} \mathrm{NMR}\left(100 \mathrm{MHz}, \mathrm{CDCl}_{3}\right) \delta(\mathrm{ppm}): 143.0,134.3,133.4,128.0,127.4,127.3,127.1,126.3$, 118.2, 102.3 78.4, 32.2, 29.9, 29.9, 29.6, 29.5, 29.4, 28.7, 23.0, 20.5, 14.4. MALDI-TOF MS (m/z): $\left[\mathrm{M}^{+}\right]$calcd for $\mathrm{C}_{42} \mathrm{H}_{52} \mathrm{~S}_{2}, 620.3510$; found 620.2775 .

\section{Synthesis of 2,2'-(1,5-di(dodecynyl)naphthalene-2,6-diyl)bis(5-chlorothiophene) (3e).}

5-Chlorothiophene-2-boronic acid pinacol ester ( $0.13 \mathrm{~g}, 0.54 \mathrm{mmol})$, compound 2c ( $0.1 \mathrm{~g}, 0.18$ $\mathrm{mmol})$, and $\mathrm{Pd}\left(\mathrm{PPh}_{3}\right)_{2} \mathrm{Cl}_{2}(0.013 \mathrm{~g}, 0.018 \mathrm{mmol})$ were dissolved in deoxygenated Tolune (4 $\mathrm{mL}$ ). The sealed tube was evacuated and backfilled with argon. Deoxygenated $2 \mathrm{M} \mathrm{K}_{2} \mathrm{CO}_{3}$ (aq) $(1 \mathrm{~mL})$ was added via syringe. The reaction mixture was stirred and heated at $85{ }^{\circ} \mathrm{C}$ overnight. After removing the solvents in vacuum, the mixture was separated and purified by column chromatography eluted with $\mathrm{PE} / \mathrm{DCM}=10 / 1$ to get a pale yellow solid $(0.35 \mathrm{~g}$, yield: $88 \%)$. ${ }^{1} \mathrm{H}$ NMR (400 MHz, $\left.\mathrm{CDCl}_{3}\right) \delta(\mathrm{ppm}): 8.36(\mathrm{~d}, J=8.8 \mathrm{~Hz}, 2 \mathrm{H}), 7.71(\mathrm{t}, J=8.8 \mathrm{~Hz}, 2 \mathrm{H}), 7.46$ $(\mathrm{d}, J=4.0 \mathrm{~Hz}, 2 \mathrm{H}), 6.96(\mathrm{t}, J=4.0 \mathrm{~Hz}, 2 \mathrm{H}), 2.66(\mathrm{t}, J=7.2 \mathrm{~Hz}, 4 \mathrm{H}), 1.78(\mathrm{~m}, 4 \mathrm{H}), 1.55(\mathrm{~m}$, $4 \mathrm{H}), 1.35(\mathrm{~m}, 16 \mathrm{H}), 0.91(\mathrm{t}, J=5.2 \mathrm{~Hz}, 6 \mathrm{H}) \cdot{ }^{13} \mathrm{C} \mathrm{NMR}\left(100 \mathrm{MHz}, \mathrm{CDCl}_{3}\right) \delta(\mathrm{ppm}): 141.3$, 133.7, 133.4, 127.2, 127.0, 126.6, 126.5, 126.3, 118.0, 103.4, 78.2, 32.1, 29.5, 29.4, 28.5, 22.9, 20.5, 20.5, 14.4. MALDI-TOF MS (m/z): $\left[\mathrm{M}^{+}\right]$calcd for $\mathrm{C}_{38} \mathrm{H}_{42} \mathrm{Cl}_{2} \mathrm{~S}_{2}, 632.2105$; found 632.1420 .

\section{Synthesis of 2,2'-(1,5-di(dodecynyl)naphthalene-2,6-diyl)bis(4-chlorothiophene) (3f).}

4-Chlorothiophene-2-boronic acid pinacol ester (0.13 g, $0.54 \mathrm{mmol}$ ), compound 2c ( $0.1 \mathrm{~g}, 0.18$ $\mathrm{mmol}), \mathrm{Pd}_{2}(\mathrm{dba})_{3}(0.0065 \mathrm{~g}, 0.007 \mathrm{mmol}), \mathrm{t}-\mathrm{Bu}_{3} \mathrm{PHBF}_{4}(0.008 \mathrm{~g}, 0.042 \mathrm{mmol})$, and $\mathrm{K}_{3} \mathrm{PO}_{4}$ $(0.25 \mathrm{~g}, 1.19 \mathrm{mmol})$ were dissolved in a degassed mixture of toluene, DMA, and water (5/5/1, $4.4 \mathrm{~mL}$ ) and stirred at $90{ }^{\circ} \mathrm{C}$ for $12 \mathrm{~h}$. After removing the solvents in vacuum, the mixture was separated and purified by column chromatography eluted with PE/DCM $=10 / 1$ to get a yellow solid (0.05 g, yield: 44\%). ${ }^{1} \mathrm{H}$ NMR (400 MHz, $\left.\mathrm{CDCl}_{3}\right) \delta(\mathrm{ppm}): 8.37$ (d, $\left.J=8.8 \mathrm{~Hz}, 2 \mathrm{H}\right), 7.71$ $(\mathrm{d}, J=8.8 \mathrm{~Hz}, 2 \mathrm{H}), 7.62(\mathrm{~d}, J=1.6 \mathrm{~Hz}, 2 \mathrm{H}), 7.18(\mathrm{t}, J=1.6 \mathrm{~Hz}, 2 \mathrm{H}), 2.64(\mathrm{t}, J=6.8 \mathrm{~Hz}, 4 \mathrm{H})$, $1.77(\mathrm{~m}, 4 \mathrm{H}), 1.53(\mathrm{~m}, 4 \mathrm{H}), 1.38(\mathrm{~m}, 16 \mathrm{H}), 0.91(\mathrm{t}, J=6.4 \mathrm{~Hz}, 6 \mathrm{H}) .{ }^{13} \mathrm{C}$ NMR $(100 \mathrm{MHz}$, $\left.\mathrm{CDCl}_{3}\right) \delta(\mathrm{ppm}): 143.2,133.5,133.3,127.6,127.3,125.4,120.7,118.6,118.5,103.2,32.1$, 29.5, 29.4, 28.6, 22.9, 20.4, 14.4. MALDI-TOF MS (m/z): $\left[\mathrm{M}^{+}\right]$calcd for $\mathrm{C}_{38} \mathrm{H}_{42} \mathrm{Cl}_{2} \mathrm{~S}_{2}$, 632.2105; found 632.1671.

Synthesis of 2,2'-(1,5-di(dodecynyl)naphthalene-2,6-diyl)bis(3-chlorothiophene) (3g). 
3-Chlorothiophene-2-boronic acid (0.088 g, $0.54 \mathrm{mmol})$, compound 2c ( $0.10 \mathrm{~g}, 0.18 \mathrm{mmol})$, and Xphos Pd G2 (0.007 g, $0.01 \mathrm{mmol})$ were dissolved in deoxygenated THF (4 mL). The sealed tube was evacuated and backfilled with argon. Deoxygenated $0.5 \mathrm{M} \mathrm{K}_{3} \mathrm{PO}_{4}(\mathrm{aq})(2 \mathrm{~mL})$ was added via syringe. The reaction mixture was stirred and heated at room temperature for 12 hours. After removing the solvents in vacuum, the mixture was separated and purified by column chromatography eluted with PE to get a pale yellow solid $(0.10 \mathrm{~g}$, yield: $88 \%) .{ }^{1} \mathrm{H}$ $\operatorname{NMR}\left(400 \mathrm{MHz}, \mathrm{CDCl}_{3}\right) \delta(\mathrm{ppm}): 8.42(\mathrm{~d}, J=8.8 \mathrm{~Hz}, 2 \mathrm{H}), 7.63(\mathrm{~d}, J=8.4 \mathrm{~Hz}, 2 \mathrm{H}), 7.39$ (d, $J=5.2 \mathrm{~Hz}, 2 \mathrm{H}), 7.04(\mathrm{~d}, J=5.2 \mathrm{~Hz}, 2 \mathrm{H}), 2.49(\mathrm{t}, J=6.8 \mathrm{~Hz}, 4 \mathrm{H}), 1.59(\mathrm{~m}, 4 \mathrm{H}), 1.36(\mathrm{~m}, 20 \mathrm{H})$, $0.91(\mathrm{~m}, 6 \mathrm{H}) .{ }^{13} \mathrm{C} \mathrm{NMR}\left(100 \mathrm{MHz}, \mathrm{CDCl}_{3}\right) \delta(\mathrm{ppm}): 135.5,133.4,133.2,129.4,128.1,126.5$, 125.2, 125.1, 124.2, 122.8, 101.6, 32.1, 29.5, 29.4, 29.1, 28.7, 22.9, 20.1, 14.4. MALDI-TOF MS (m/z): $\left[\mathrm{M}^{+}\right]$calcd for $\mathrm{C}_{38} \mathrm{H}_{42} \mathrm{Cl}_{2} \mathrm{~S}_{2}, 632.2105$; found 632.1060 .

\section{Synthesis of 7,14-dibutylnaphtho[2,1-f:6,5-f']bis(cyclopenta[b]thiopyran) (C4-SS).}

To a degassed solution of 3a $(0.20 \mathrm{~g}, 0.44 \mathrm{mmol})$ in dry toluene $(20 \mathrm{~mL})$ was added $\mathrm{PtCl}_{2}$ $(0.024 \mathrm{~g}, 0.088 \mathrm{mmol})$. The mixture was heated overnight under $110{ }^{\circ} \mathrm{C}$ and then cooled to room temperature. After removing toluene in vacuum, the product was separated and purified through column chromatography (eluted with $\mathrm{PE} / \mathrm{DCM}=5 / 1$ ) to give $\mathbf{C 4 - S S}$ as dark green crystals (0.10 g, yield: $50 \%) .{ }^{1} \mathrm{H}$ NMR (400 MHz, $\left.\mathrm{CD}_{2} \mathrm{Cl}_{2}\right) \delta(\mathrm{ppm}): 8.53(\mathrm{~d}, J=8.8 \mathrm{~Hz}, 2 \mathrm{H})$, $8.35(\mathrm{~d}, J=8.8 \mathrm{~Hz}, 2 \mathrm{H}), 8.09(\mathrm{~d}, J=6.8 \mathrm{~Hz}, 2 \mathrm{H}), 7.65(\mathrm{~d}, J=8.8 \mathrm{~Hz}, 2 \mathrm{H}), 7.21(\mathrm{t}, J=7.2,2 \mathrm{H})$, $3.39(\mathrm{t}, J=7.6,4 \mathrm{H}), 1.95(\mathrm{~m}, 4 \mathrm{H}), 1.63(\mathrm{~m}, 4 \mathrm{H}), 1.05(\mathrm{t}, J=7.2 \mathrm{~Hz}, 6 \mathrm{H}) .{ }^{13} \mathrm{C}$ NMR $(100 \mathrm{MHz}$, $\left.\mathrm{CD}_{2} \mathrm{Cl}_{2}\right) \delta(\mathrm{ppm}): 137.3,130.4,128.5,127.5,126.7,126.4,123.5,123.2,118.3,118.1,117.3$, 31.3, 29.4, 23.2, 14.1. MALDI-TOF MS (m/z): [ $\left.\mathrm{M}^{+}\right]$calcd for $\mathrm{C}_{30} \mathrm{H}_{28} \mathrm{~S}_{2}$, 452.1632; found 452.1125 .

\section{Synthesis of 7,14-dihexylnaphtho[2,1-f:6,5-f']bis(cyclopenta[b]thiopyran) (C6-SS).}

To a degassed solution of $\mathbf{3 b}(0.23 \mathrm{~g}, 0.45 \mathrm{mmol})$ in dry toluene $(23 \mathrm{~mL})$ was added $\mathrm{PtCl}_{2}$ $(0.024 \mathrm{~g}, 0.091 \mathrm{mmol})$. The mixture was heated overnight under $110{ }^{\circ} \mathrm{C}$ and then cooled to room temperature. After removing toluene in vacuum, the product was separated and purified through column chromatography (eluted with PE/DCM $=6 / 1$ ) to give C6-SS as dark green crystals (0.14 g, yield: 60\%). ${ }^{1} \mathrm{H}$ NMR (400 MHz, $\left.\mathrm{CD}_{2} \mathrm{Cl}_{2}\right) \delta(\mathrm{ppm}): 8.52(\mathrm{~d}, J=8.4 \mathrm{~Hz}, 2 \mathrm{H})$, $8.35(\mathrm{~d}, J=8.4 \mathrm{~Hz}, 2 \mathrm{H}), 8.10(\mathrm{~d}, J=7.2 \mathrm{~Hz}, 2 \mathrm{H}), 7.65(\mathrm{~d}, J=9.2 \mathrm{~Hz}, 2 \mathrm{H}), 7.22(\mathrm{t}, J=8.0 \mathrm{~Hz}$, 2H), $3.38(\mathrm{t}, J=7.6 \mathrm{~Hz}, 4 \mathrm{H}), 1.96(\mathrm{~m}, 4 \mathrm{H}), 1.63(\mathrm{~m}, 4 \mathrm{H}), 1.44(\mathrm{~m}, 8 \mathrm{H}), 0.93(\mathrm{t}, J=6.8 \mathrm{~Hz}$, $6 \mathrm{H}) .{ }^{13} \mathrm{C}$ NMR peaks were barely detected because of the low solubility of $\mathbf{C}_{6}-\mathbf{S S}$ in deuterated solvent. MALDI-TOF MS (m/z): [M+] calcd for $\mathrm{C}_{34} \mathrm{H}_{36} \mathrm{~S}_{2}, 508.2258$; found 508.1645. 


\section{Synthesis of 7,14-dioctylnaphtho[2,1-f:6,5-f']bis(cyclopenta[b]thiopyran) (C8-SS).}

To a degassed solution of $\mathbf{3 c}(0.62 \mathrm{~g}, 1.10 \mathrm{mmol})$ in dry toluene $(60 \mathrm{~mL})$ was added $\mathrm{PtCl}_{2}(0.06$ $\mathrm{g}, 0.22 \mathrm{mmol}$ ). The mixture was heated overnight under $110{ }^{\circ} \mathrm{C}$ and then cooled to room temperature. After removing toluene in vacuum, the product was separated and purified through column chromatography (eluted with PE/DCM = 30/1) to give $\mathbf{C 8}$-SS as dark green crystals (0.32 g, yield: $51 \%) .{ }^{1} \mathrm{H}$ NMR (400 MHz, $\left.\mathrm{CD}_{2} \mathrm{Cl}_{2}\right) \delta(\mathrm{ppm}): 8.52$ (d, $\left.J=7.6 \mathrm{~Hz}, 2 \mathrm{H}\right)$, $8.34(\mathrm{~d}, J=8.4 \mathrm{~Hz}, 2 \mathrm{H}), 8.09(\mathrm{~d}, J=7.2 \mathrm{~Hz}, 2 \mathrm{H}), 7.65(\mathrm{~d}, J=9.2 \mathrm{~Hz}, 2 \mathrm{H}), 7.22(\mathrm{t}, J=8.0,2 \mathrm{H})$, $3.36(\mathrm{t}, J=8.4,4 \mathrm{H}), 1.96(\mathrm{~m}, 4 \mathrm{H}), 1.63(\mathrm{~m}, 4 \mathrm{H}), 1.44(\mathrm{~m}, 4 \mathrm{H}), 1.35(\mathrm{~m}, 12 \mathrm{H}), 0.90(\mathrm{t}, J=5.2$ $\mathrm{Hz}, 6 \mathrm{H}) .{ }^{13} \mathrm{C} \mathrm{NMR}\left(100 \mathrm{MHz}, \mathrm{CDCl}_{3}\right) \delta$ (ppm): 137.4, 130.6, 128.4, 127.3, 126.4, 126.3, 123.3, 122.8, 118.3, 118.1, 117.2, 32.1, 30.1, 29.9, 29.8, 29.6, 29.0, 22.9, 14.3. MALDI-TOF MS $(\mathrm{m} / \mathrm{z}):[\mathrm{M}+\mathrm{H}]^{+}$calcd for $\mathrm{C}_{38} \mathrm{H}_{45} \mathrm{~S}_{2} 565.2963$; found 565.2965.

\section{Synthesis of 7,14-didecylnaphtho[2,1-f:6,5- $\left.f^{\prime}\right]$ bis(cyclopenta[b]thiopyran) (C10-SS).}

To a degassed solution of $\mathbf{3 d}(0.10 \mathrm{~g}, 0.16 \mathrm{mmol})$ in dry toluene $(10 \mathrm{~mL})$ was added $\mathrm{PtCl}_{2}$ $(0.010 \mathrm{~g}, 0.032 \mathrm{mmol})$. The mixture was heated overnight under $110{ }^{\circ} \mathrm{C}$ and then cooled to room temperature. After removing toluene in vacuum, the product was separated and purified through column chromatography (eluted with PE/DCM = 30/1) to give $\mathbf{C} \mathbf{1 0}-\mathbf{S S}$ as dark green crystals (0.042 g, yield: 42\%). ${ }^{1} \mathrm{H}$ NMR (400 MHz, $\left.\mathrm{CDCl}_{3}\right) \delta(\mathrm{ppm}): 8.52(\mathrm{~d}, J=8.8 \mathrm{~Hz}, 2 \mathrm{H})$, $8.32(\mathrm{~d}, J=8.4 \mathrm{~Hz}, 2 \mathrm{H}), 8.04(\mathrm{~d}, J=7.2 \mathrm{~Hz}, 2 \mathrm{H}), 7.59$ (s, 2H), 7.19 (t, $J=8.8,2 \mathrm{H}), 3.37$ (t, $J$ $=8.4,4 \mathrm{H}), 1.93(\mathrm{~m}, 4 \mathrm{H}), 1.41-1.26(\mathrm{~m}, 28 \mathrm{H}), 0.87(\mathrm{t}, J=6.8 \mathrm{~Hz}, 6 \mathrm{H}) .{ }^{13} \mathrm{C} \mathrm{NMR}(100 \mathrm{MHz}$, $\left.\mathrm{C}_{2} \mathrm{D}_{2} \mathrm{Cl}_{4}\right) \delta$ (ppm): 137.6, 130.8, 128.6, 127.2, 126.5, 123.3, 122.6, 118.3, 118.2, 117.3, 32.0, 30.1, 29.7, 29.3, 28.8, 22.7, 14.0. MALDI-TOF MS (m/z): $\left[\mathrm{M}^{+}\right]$calcd for $\mathrm{C}_{42} \mathrm{H}_{52} \mathrm{~S}_{2}, 620.3510$; found 620.2712 .

\section{Synthesis of 5,12-dichloro-7,14-dioctylnaphtho[2,1-f:6,5- $\left.f^{\prime}\right]$ bis(cyclopenta[b]thiopyran) $\left(\mathrm{C}_{8}-\mathrm{SS}-\mathrm{Cl}^{2}\right)$.}

To a degassed solution of $\mathbf{3 e}(0.2 \mathrm{~g}, 0.32 \mathrm{mmol})$ in dry toluene $(20 \mathrm{~mL})$ was added $\mathrm{PtCl}_{2}(0.03$ $\mathrm{g}, 0.13 \mathrm{mmol})$. The mixture was heated overnight under $110{ }^{\circ} \mathrm{C}$ and then cooled to room temperature. After removing toluene in vacuum, the product was separated and purified

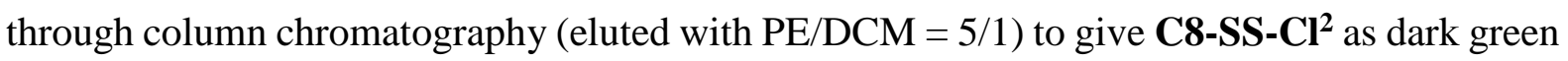
crystals (0.32 g, yield: $51 \%) .{ }^{1} \mathrm{H}$ NMR (400 MHz, $\left.\mathrm{CD}_{2} \mathrm{Cl}_{2}\right) \delta(\mathrm{ppm}): 8.45$ (d, $\left.J=8.4 \mathrm{~Hz}, 2 \mathrm{H}\right)$, $8.24(\mathrm{~d}, J=8.4 \mathrm{~Hz}, 2 \mathrm{H}), 7.85(\mathrm{~d}, J=8.0 \mathrm{~Hz}, 2 \mathrm{H}), 7.20(\mathrm{~d}, J=8.0 \mathrm{~Hz}, 2 \mathrm{H}), 3.28(\mathrm{t}, J=7.6,4 \mathrm{H})$, 1.43-1.28 (m, 24H), $0.91(\mathrm{t}, J=6.4 \mathrm{~Hz}, 6 \mathrm{H}) .{ }^{13} \mathrm{C}$ NMR peaks were barely detected because of 
the low solubility of $\mathbf{C s}_{\mathbf{8}} \mathbf{S S}-\mathbf{C l}^{2}$ in deuterated solvent. MALDI-TOF MS (m/z): $\left[\mathrm{M}^{+}\right]$calcd for $\mathrm{C}_{38} \mathrm{H}_{42} \mathrm{Cl}_{2} \mathrm{~S}_{2}, 632.2105$; found 632.1396 .

\section{Synthesis of 4,11-dichloro-7,14-dioctylnaphtho[2,1-f:6,5- $\left.f^{\prime}\right]$ bis(cyclopenta[b]thiopyran) $\left(\mathrm{C}_{8}-\mathrm{SS}-\mathrm{Cl}^{3}\right)$.}

To a degassed solution of $\mathbf{3 f}(0.06 \mathrm{~g}, 0.095 \mathrm{mmol})$ in dry toluene $(6 \mathrm{~mL})$ was added $\mathrm{PtCl}_{2}(0.01$ $\mathrm{g}, 0.04 \mathrm{mmol})$. The mixture was heated overnight under $110{ }^{\circ} \mathrm{C}$ and then cooled to room temperature. After removing toluene in vacuum, the product was separated and purified through column chromatography (eluted with $\mathrm{PE} / \mathrm{DCM}=4 / 1$ ) to give $\mathbf{C} \mathbf{8}-\mathbf{S S}-\mathbf{C l}^{\mathbf{3}}$ as dark green crystals (0.02 g, yield: 33\%). ${ }^{1} \mathrm{H}$ NMR (400 MHz, $\left.\mathrm{CDCl}_{3}\right) \delta$ (ppm): 8.39 (d, $\left.J=8.4 \mathrm{~Hz}, 2 \mathrm{H}\right)$, $8.18(\mathrm{~d}, J=8.4 \mathrm{~Hz}, 2 \mathrm{H}), 7.77(\mathrm{~s}, 2 \mathrm{H}), 7.45(\mathrm{~s}, 2 \mathrm{H}), 3.27(\mathrm{t}, J=7.6,4 \mathrm{H}), 1.9(\mathrm{~m}, 4 \mathrm{H}), 1.57$ (m, $4 \mathrm{H}), 1.41-1.25(\mathrm{~m}, 16 \mathrm{H}), 0.90(\mathrm{t}, J=6.7 \mathrm{~Hz}, 6 \mathrm{H}) .{ }^{13} \mathrm{C} \mathrm{NMR}\left(100 \mathrm{MHz}, \mathrm{CDCl}_{3}\right) \delta(\mathrm{ppm}): 138.3$, 133.9, 128.7, 127.8, 126.2, 124.5, 121.7, 121.6, 121.2 , 120.7, 118.6, 32.2, 30.1, 30.0, 29.8, 29.6, 28.9, 22.9, 14.4. MALDI-TOF MS (m/z): $\left[\mathrm{M}^{+}\right]$calcd for $\mathrm{C}_{38} \mathrm{H}_{42} \mathrm{Cl}_{2} \mathrm{~S}_{2}, 632.2105$; found 632.0798 .

\section{Synthesis of 3,10-dichloro-7,14-dioctylnaphtho[2,1-f:6,5- $\left.f^{\prime}\right]$ bis(cyclopenta[b]thiopyran) $\left(\mathrm{C}_{8}-\mathrm{SS}-\mathrm{Cl}^{4}\right)$.}

To a degassed solution of $\mathbf{3 g}(0.08 \mathrm{~g}, 0.13 \mathrm{mmol})$ in dry toluene $(8 \mathrm{~mL})$ was added $\mathrm{PtCl}_{2}(0.013$ $\mathrm{g}, 0.051 \mathrm{mmol})$. The mixture was heated overnight under $110^{\circ} \mathrm{C}$ and then cooled to room temperature. After removing toluene in vacuum, the product was separated and purified through column chromatography (eluted with $\mathrm{PE} / \mathrm{DCM}=5 / 1$ ) to give $\mathbf{C 8}-\mathbf{S S}-\mathbf{C l}^{\mathbf{4}}$ as dark green crystals (0.04 g, yield: 50\%). ${ }^{1} \mathrm{H}$ NMR (400 MHz, THF- $\left.d_{8}\right) \delta(\mathrm{ppm}): 8.99$ (d, $J=8.8 \mathrm{~Hz}, 2 \mathrm{H}$ ), $8.59(\mathrm{~d}, J=8.8 \mathrm{~Hz}, 2 \mathrm{H}), 7.91(\mathrm{~d}, J=9.6 \mathrm{~Hz}, 2 \mathrm{H}), 7.21(\mathrm{~d}, J=9.6 \mathrm{~Hz}, 2 \mathrm{H}), 3.42(\mathrm{t}, J=7.2,4 \mathrm{H})$, $1.96(\mathrm{~m}, 4 \mathrm{H}), 1.63(\mathrm{~m}, 4 \mathrm{H}), 1.45-1.32(\mathrm{~m}, 16 \mathrm{H}), 0.91(\mathrm{t}, J=6.7 \mathrm{~Hz}, 6 \mathrm{H}) .{ }^{13} \mathrm{C}$ NMR peaks were barely detected because of the low solubility of $\mathbf{C s - S S - \mathbf { C l } ^ { 4 }}$ in deuterated solvent. MALDI-TOF MS (m/z): $\left[\mathrm{M}^{+}\right]$calcd for $\mathrm{C}_{38} \mathrm{H}_{42} \mathrm{Cl}_{2} \mathrm{~S}_{2}, 632.2105$; found 632.1501 . 


\section{X-ray crystallographic data}

Table S1. X-ray crystal data and structure refinement details for $\mathbf{C}_{\mathbf{n}-S S}(\mathrm{n}=4,6,8,10)$ and $\mathbf{C}$-SS-CI ${ }^{\mathbf{m}}(\mathrm{m}=2,3,4)$.

\begin{tabular}{|c|c|c|c|c|c|c|c|c|c|c|}
\hline & $\mathrm{C}_{4}-\mathrm{SS}$ & $\mathrm{C}_{6}-\mathrm{SS}$ & $\mathrm{C}_{8}-\mathrm{SS}$ & $\mathrm{C}_{10-\mathrm{SS}}$ & $\mathrm{C}_{8}-\mathrm{SS}_{-} \mathrm{Cl}^{2}$ (I) & $\mathrm{C}_{8}-\mathrm{SS}_{-} \mathrm{Cl}^{2}$ (II) & $\mathrm{C}_{8}-\mathrm{SS}_{-}-\mathrm{Cl}^{3}(\mathrm{I})$ & $\mathrm{C}_{8}-\mathrm{SS}_{-} \mathrm{Cl}^{3}$ (II) & $\mathrm{C}_{8}-\mathrm{SS}_{-}-\mathrm{Cl}^{4}$ (I) & ${\mathrm{C} 8-\mathrm{SS}-\mathrm{Cl}^{4}}^{4}$ (II) \\
\hline CCDC No. & 2079886 & 2079887 & 1579137 & 2079888 & 2079889 & 2079890 & 2079892 & 2079893 & 2079896 & 2079897 \\
\hline formula & $\mathrm{C}_{30} \mathrm{H}_{29} \mathrm{~S}_{2}$ & $\mathrm{C}_{34} \mathrm{H}_{37} \mathrm{~S}_{2}$ & $\mathrm{C}_{38} \mathrm{H}_{44} \mathrm{~S}_{2}$ & $\mathrm{C}_{42} \mathrm{H}_{53} \mathrm{~S}_{2}$ & $\mathrm{C}_{38} \mathrm{H}_{42} \mathrm{Cl}_{2} \mathrm{~S}_{2}$ & $\mathrm{C}_{38} \mathrm{H}_{42} \mathrm{Cl}_{2} \mathrm{~S}_{2}$ & $\mathrm{C}_{38} \mathrm{H}_{42} \mathrm{Cl}_{2} \mathrm{~S}_{2}$ & $\mathrm{C}_{38} \mathrm{H}_{42} \mathrm{Cl}_{2} \mathrm{~S}_{2}$ & $\mathrm{C}_{38} \mathrm{H}_{42} \mathrm{Cl}_{2} \mathrm{~S}_{2}$ & $\mathrm{C}_{38} \mathrm{H}_{42} \mathrm{Cl}_{2} \mathrm{~S}_{2}$ \\
\hline formula wt. & 452.64 & 508.75 & 564.85 & 620.95 & 633.73 & 633.73 & 633.73 & 633.73 & 633.73 & 633.73 \\
\hline$T(\mathrm{~K})$ & 173 & 173 & 173 & 173 & 173 & 173 & 173 & 173 & 173 & 173 \\
\hline wavelength $(\AA)$ & 1.34138 & 1.34138 & 1.54178 & 1.34138 & 1.34138 & 1.34138 & 1.34138 & 1.54178 & 1.34138 & 1.54178 \\
\hline crystal size & $\begin{array}{l}0.124 \times 0.097 \\
\times 0.057\end{array}$ & $\begin{array}{l}0.37 \times 0.32 \times \\
0.02\end{array}$ & $\begin{array}{l}0.16 \times 0.15 \times \\
0.12\end{array}$ & $\begin{array}{l}0.38 \times 0.12 \times \\
0.022\end{array}$ & $\begin{array}{l}0.16 \times 0.14 \times \\
0.02\end{array}$ & $\begin{array}{l}0.21 \times 0.13 \times \\
0.04\end{array}$ & $\begin{array}{l}0.2 \times 0.05 \times \\
0.02\end{array}$ & $\begin{array}{l}0.22 \times 0.20 \times \\
0.18\end{array}$ & $\begin{array}{l}0.17 \times 0.07 \times \\
0.06\end{array}$ & $\begin{array}{l}0.22 \times 0.20 \times \\
0.18\end{array}$ \\
\hline crystal syst. & Triclinic & Monoclinic & Triclinic & Triclinic & Monoclinic & Triclinic & Monoclinic & Monoclinic & Triclinic & Monoclinic \\
\hline space group & $P \overline{1}$ & $P 2_{1} / \mathrm{c}$ & $P \overline{1}$ & $P \overline{1}$ & $P 2{ }_{1}$ & $P \overline{1}$ & $C 2 / \mathrm{c}$ & $C 2 / \mathrm{c}$ & $P \overline{1}$ & $C 2 / \mathrm{c}$ \\
\hline$a(\AA)$ & $5.1422(2)$ & $15.7030(8)$ & $4.9037(8)$ & $4.9026(4)$ & $10.7920(4)$ & $10.6210(8)$ & $36.575(5)$ & $36.335(13)$ & $9.8065(9)$ & $31.548(11)$ \\
\hline$b(\AA)$ & $9.0176(3)$ & $9.0532(4)$ & $9.209(3)$ & $9.1859(8)$ & $8.5941(3)$ & $18.0327(13)$ & $4.6603(5)$ & $4.7001(12)$ & $9.8987(10)$ & $6.284(2)$ \\
\hline$c(\AA)$ & $12.6265(4)$ & $9.2855(5)$ & $17.287(5)$ & $19.6608(18)$ & $18.0287(7)$ & $18.5604(13)$ & $25.037(3)$ & $24.950(10)$ & $10.0107(11)$ & $21.939(8)$ \\
\hline$\alpha$ (deg.) & $98.5390(10)$ & 90 & $78.265(16)$ & $77.459(3)$ & 90 & $98.576(3)$ & 90 & 90 & $105.185(4)$ & 90 \\
\hline$\beta$ (deg.) & $100.4140(10)$ & $90.936(2)$ & $87.365(18)$ & $84.069(4)$ & $90.812(1)$ & $97.107(4)$ & $120.487(6)$ & $119.852(16)$ & $97.456(4)$ & $131.761(15)$ \\
\hline$\gamma($ deg. $)$ & $97.7600(10)$ & 90 & $80.485(15)$ & $80.555(4)$ & 90 & $104.089(3)$ & 90 & 90 & $117.295(4)$ & 90 \\
\hline$V\left(\AA^{3}\right)$ & $561.53(3)$ & $1319.87(11)$ & $753.8(3)$ & $850.47(13)$ & $1671.95(11)$ & $3361.5(4)$ & $3677.6(8)$ & $3696(2)$ & $797.25(14)$ & $3244(2)$ \\
\hline $\mathrm{Z} / \mathrm{D}_{\text {calcd. }}\left(\mathrm{mg} / \mathrm{m}^{3}\right)$ & $1 / 1.339$ & $2 / 1.280$ & $1 / 1.244$ & $1 / 1.212$ & $2 / 1.259$ & 4/ 1.252 & $4 / 1.145$ & $4 / 1.139$ & $1 / 1.32$ & 4/ 1.298 \\
\hline$\mu\left(\mathrm{mm}^{-1}\right)$ & 1.481 & 1.296 & 1.776 & 1.060 & 2.046 & 2.036 & 1.861 & 2.799 & 2.146 & 3.189 \\
\hline$F(000)$ & 240 & 544 & 304 & 336 & 672 & 1344 & 1344 & 1344 & 336 & 1344 \\
\hline $\begin{array}{l}\max / \min \\
\text { transmission }\end{array}$ & $0.752 / 0.606$ & $0.751 / 0.487$ & $0.753 / 0.698$ & $0.752 / 0.542$ & $0.751 / 0.568$ & $0.751 / 0.564$ & $0.751 / 0.593$ & $0.753 / 0.522$ & $0.752 / 0.563$ & $0.751 / 0.716$ \\
\hline $\begin{array}{l}\text { final } R \text { indices } \\
{[I>2 \theta(I)]}\end{array}$ & $\begin{array}{l}R 1=0.0323 \\
w R 2=0.0820\end{array}$ & $\begin{array}{l}R 1=0.0419 \\
w R 2=0.1143\end{array}$ & $\begin{array}{l}R 1=0.0354 \\
w R 2=0.0800\end{array}$ & $\begin{array}{l}R 1=0.0381 \\
w R 2=0.1081\end{array}$ & $\begin{array}{l}R 1=0.0337 \\
w R 2=0.0872\end{array}$ & $\begin{array}{l}R 1=0.0472 \\
w R 2=0.1253\end{array}$ & $\begin{array}{l}R 1=0.0558 \\
w R 2=0.1469\end{array}$ & $\begin{array}{l}R 1=0.0543 \\
w R 2=0.1532\end{array}$ & $\begin{array}{l}R 1=0.0369 \\
w R 2=0.0812\end{array}$ & $\begin{array}{l}R 1=0.0317 \\
w R 2=0.0795\end{array}$ \\
\hline \multirow[t]{2}{*}{$\begin{array}{l}\mathrm{R} \text { indices (all } \\
\text { data) }\end{array}$} & $R=0.0341$ & $R=0.0424$ & $R=0.0411$ & $R=0.039$ & $R=0.0374$ & $R=0.066$ & $R=0.0874$ & $R=0.0678$ & $R=0.0464$ & $R=0.0396$ \\
\hline & $w R 2=0.0835$ & $w R 2=0.1149$ & $w R 2=0.0824$ & $w R 2=0.1091$ & $w R 2=0.0896$ & $w R 2=0.1350$ & $w R 2=0.1701$ & $w R 2=0.1624$ & $w R 2=0.0867$ & $w R 2=0.0857$ \\
\hline
\end{tabular}


Table S2. X-ray crystal data and structure refinement details for $\mathbf{C}_{8}-\mathbf{S S}-\mathbf{C l}^{2}$ crystallized from different solvents.

\begin{tabular}{|c|c|c|c|c|}
\hline solvent & Chloroform & Tetrachloroethane & Chlorobenzene & $o$-Dichlorobenzene \\
\hline CCDC No. & 2079889 & 2088966 & 2079890 & 2088965 \\
\hline formula & $\mathrm{C}_{38} \mathrm{H}_{42} \mathrm{Cl}_{2} \mathrm{~S}_{2}$ & $\mathrm{C}_{38} \mathrm{H}_{42} \mathrm{Cl}_{2} \mathrm{~S}_{2}$ & $\mathrm{C}_{38} \mathrm{H}_{42} \mathrm{Cl}_{2} \mathrm{~S}_{2}$ & $\mathrm{C}_{38} \mathrm{H}_{42} \mathrm{Cl}_{2} \mathrm{~S}_{2}$ \\
\hline formula wt. & 633.73 & 633.73 & 633.73 & 633.73 \\
\hline$T(\mathrm{~K})$ & 173 & 173 & 173 & 173 \\
\hline wavelength $(\AA)$ & 1.34138 & 1.54178 & 1.34138 & 1.54178 \\
\hline crystal size & $\begin{array}{l}0.16 \times 0.14 \\
\times 0.02\end{array}$ & $0.16 \times 0.15 \times 0.12$ & $\begin{array}{l}0.21 \times 0.13 \times \\
0.04\end{array}$ & $0.15 \times 0.14 \times 0.12$ \\
\hline crystal syst. & Monoclinic & Monoclinic & Triclinic & Triclinic \\
\hline space group & $P 2_{1}$ & $P 2_{1}$ & $P \overline{1}$ & $P \overline{1}$ \\
\hline$a(\AA)$ & $10.7920(4)$ & $10.7988(2)$ & $10.6210(8)$ & $10.6984(11)$ \\
\hline$b(\AA)$ & $8.5941(3)$ & $8.6085(2)$ & $18.0327(13)$ & $17.9655(16)$ \\
\hline$c(\AA)$ & $18.0287(7)$ & $18.0304(4)$ & $18.5604(13)$ & $18.502(2)$ \\
\hline$\alpha$ (deg.) & 90 & 90 & $98.576(3)$ & $97.906(6)$ \\
\hline$\beta$ (deg.) & $90.812(1)$ & $90.8780(10)$ & $97.107(4)$ & $97.068(6)$ \\
\hline$\gamma($ deg. $)$ & 90 & 90 & $104.089(3)$ & $104.531(10)$ \\
\hline$V\left(\AA^{3}\right)$ & $1671.95(11)$ & $1675.94(6)$ & $3361.5(4)$ & $3363.3(6)$ \\
\hline $\mathrm{Z} / \mathrm{D}_{\text {calcd. }}\left(\mathrm{mg} / \mathrm{m}^{3}\right)$ & $2 / 1.259$ & $2 / 1.256$ & 4/ 1.252 & 4/ 1.252 \\
\hline$\mu\left(\mathrm{mm}^{-1}\right)$ & 2.046 & 3.086 & 2.036 & 3.076 \\
\hline$F(000)$ & 672 & 672 & 1344 & 1344 \\
\hline $\begin{array}{l}\max / \min \\
\text { transmission }\end{array}$ & $0.751 / 0.568$ & $0.753 / 0.552$ & $0.751 / 0.564$ & $0.753 / 0.546$ \\
\hline $\begin{array}{l}\text { final } R \text { indices } \\
{[I>2 \theta(I)]}\end{array}$ & $\begin{array}{l}R 1=0.0337 \\
w R 2= \\
0.0872\end{array}$ & $\begin{array}{l}R 1=0.0370 \\
w R 2=0.1018\end{array}$ & $\begin{array}{l}R 1=0.0472 \\
w R 2=0.1253\end{array}$ & $\begin{array}{l}R 1=0.0384 \\
w R 2=0.0977\end{array}$ \\
\hline \multirow[t]{2}{*}{$\mathrm{R}$ indices (all data) } & $R=0.0374$ & $R=0.0427$ & $R=0.066$ & $R=0.0476$ \\
\hline & $\begin{array}{l}w R 2= \\
0.0896\end{array}=$ & $w R 2=0.1067$ & $w R 2=0.1350$ & $w R 2=0.1035$ \\
\hline
\end{tabular}


Table S3. Selected bond lengths for $\mathbf{C}_{\mathbf{4}}$-SS .

\begin{tabular}{cc}
\hline Bond & Length/A \\
\hline S1-C1 & $1.7171(15)$ \\
S1-C5 & $1.7351(13)$ \\
C1-C2 & $1.3510(20)$ \\
C2-C3 & $1.4318(19)$ \\
C3-C4 & $1.3566(18)$ \\
C4-C8 & $1.4451(18)$ \\
C4-C5 & $1.4659(17)$ \\
C5-C6 & $1.3654(18)$ \\
C6-C7 & $1.4710(17)$ \\
C6-C12 & $1.5044(17)$ \\
C7-C8 & $1.4077(17)$ \\
C7-C11 & $1.4316(17)$ \\
C9-C10 & $1.3653(18)$ \\
C9-C8 & $1.4025(17)$ \\
C10-C11 & $1.4261(17)$ \\
C11-C11 & $1.4460(20)$ \\
C12-C13 & $1.5355(18)$ \\
C13-C14 & $1.5200(20)$ \\
C14-C15 & $1.5210(20)$ \\
\hline
\end{tabular}


Table S4. Selected bond lengths for $\mathbf{C}_{6}-\mathbf{S S}$.

\begin{tabular}{cc}
\hline Bond & Length/A \\
\hline S1-C1 & $1.7230(15)$ \\
S1-C5 & $1.7275(14)$ \\
C1-C2 & $1.3440(20)$ \\
C2-C3 & $1.4350(19)$ \\
C3-C4 & $1.3579(19)$ \\
C4-C8 & $1.4451(18)$ \\
C4-C5 & $1.4664(18)$ \\
C5-C6 & $1.3703(19)$ \\
C6-C7 & $1.4678(18)$ \\
C6-C12 & $1.5018(18)$ \\
C7-C8 & $1.4044(18)$ \\
C7-C11 & $1.4353(18)$ \\
C9-C10 & $1.3688(18)$ \\
C9-C8 & $1.4066(18)$ \\
C10-C11 & $1.4200(20)$ \\
C11-C11 & $1.4470(20)$ \\
C12-C13 & $1.5363(19)$ \\
C13-C14 & $1.5223(19)$ \\
C14-C15 & $1.5225(19)$ \\
C15-C16 & $1.5255(19)$ \\
C16-C17 & $1.5170(20)$ \\
\hline
\end{tabular}


Table S5. Selected bond lengths for $\mathbf{C}_{8}-\mathbf{S S}$.

\begin{tabular}{cc}
\hline Bond & Length/A \\
\hline S1-C1 & $1.7158(18)$ \\
S1-C5 & $1.7376(17)$ \\
C1-C2 & $1.3480(30)$ \\
C2-C3 & $1.4280(20)$ \\
C3-C4 & $1.3510(20)$ \\
C4-C8 & $1.4400(20)$ \\
C4-C5 & $1.4640(20)$ \\
C5-C6 & $1.3600(20)$ \\
C6-C7 & $1.4720(20)$ \\
C6-C12 & $1.5040(20)$ \\
C7-C8 & $1.4050(20)$ \\
C7-C11 & $1.4280(20)$ \\
C9-C10 & $1.3610(20)$ \\
C9-C8 & $1.4050(20)$ \\
C10-C11 & $1.4200(20)$ \\
C11-C7 & $1.4280(20)$ \\
C11-C11 & $1.4440(30)$ \\
C12-C13 & $1.5370(20)$ \\
C13-C14 & $1.5200(20)$ \\
C14-C15 & $1.5260(20)$ \\
C15-C16 & $1.5190(20)$ \\
C16-C17 & $1.5190(30)$ \\
C17-C18 $180(20)$ \\
C18-C19 $1.5210(30)$ \\
\hline
\end{tabular}


Table S6. Selected bond lengths for $\mathbf{C}_{10}-\mathbf{S S}$.

\begin{tabular}{|c|c|}
\hline Bond & Length/Å \\
\hline $\mathrm{S} 1-\mathrm{C} 1$ & $1.7187(13)$ \\
\hline S1-C5 & $1.7302(12)$ \\
\hline $\mathrm{C} 1-\mathrm{C} 2$ & $1.3495(19)$ \\
\hline $\mathrm{C} 2-\mathrm{C} 3$ & $1.4298(18)$ \\
\hline $\mathrm{C} 3-\mathrm{C} 4$ & $1.3584(16)$ \\
\hline $\mathrm{C} 4-\mathrm{C} 8$ & $1.4433(16)$ \\
\hline C4-C5 & $1.4670(16)$ \\
\hline C5-C6 & $1.3687(16)$ \\
\hline C6-C7 & $1.4706(15)$ \\
\hline C6-C12 & $1.5037(15)$ \\
\hline $\mathrm{C} 7-\mathrm{C} 8$ & $1.4082(14)$ \\
\hline C7-C11 & $1.4295(16)$ \\
\hline C9-C10 & $1.3650(16)$ \\
\hline C9-C8 & $1.4042(16)$ \\
\hline C10-C11 & $1.4261(14)$ \\
\hline C11-C11 & $1.4420(20)$ \\
\hline C12-C13 & $1.5356(15)$ \\
\hline C13-C14 & $1.5223(16)$ \\
\hline C14-C15 & $1.5257(16)$ \\
\hline C15-C16 & $1.5248(17)$ \\
\hline C16-C17 & $1.5242(17)$ \\
\hline C17-C18 & $1.5249(17)$ \\
\hline C18-C19 & $1.5230(18)$ \\
\hline C19-C20 & $1.5221(19)$ \\
\hline C20-C21 & $1.5200(20)$ \\
\hline
\end{tabular}


Table S7. Selected bond lengths for $\mathbf{C}_{8}-\mathrm{SS}_{-}-\mathbf{C l}^{2}$ (I).

\begin{tabular}{|c|c|}
\hline Bond & Length/Å \\
\hline $\mathrm{S} 1-\mathrm{C} 1$ & $1.736(3)$ \\
\hline S2-C18 & $1.728(3)$ \\
\hline S2-C22 & $1.729(3)$ \\
\hline C11-C1 & $1.727(3)$ \\
\hline $\mathrm{C} 12-\mathrm{C} 22$ & $1.733(3)$ \\
\hline $\mathrm{C} 1-\mathrm{C} 2$ & $1.342(4)$ \\
\hline $\mathrm{C} 2-\mathrm{C} 3$ & $1.428(4)$ \\
\hline $\mathrm{C} 3-\mathrm{C} 4$ & $1.357(4)$ \\
\hline $\mathrm{C} 4-\mathrm{C} 8$ & $1.449(4)$ \\
\hline $\mathrm{C} 4-\mathrm{C} 5$ & $1.456(4)$ \\
\hline C5-C6 & $1.368(4)$ \\
\hline C6-C7 & $1.472(4)$ \\
\hline C6-C23 & $1.497(4)$ \\
\hline C7-C8 & $1.401(3)$ \\
\hline C7-C12 & $1.430(4)$ \\
\hline $\mathrm{C} 8-\mathrm{C} 9$ & $1.405(4)$ \\
\hline C9-C10 & $1.359(4)$ \\
\hline $\mathrm{C} 10-\mathrm{C} 11$ & $1.429(3)$ \\
\hline C11-C16 & $1.427(4)$ \\
\hline $\mathrm{C} 11-\mathrm{C} 12$ & $1.448(3)$ \\
\hline C12-C13 & $1.421(3)$ \\
\hline C14-C15 & $1.400(4)$ \\
\hline $\mathrm{C} 15-\mathrm{C} 16$ & $1.407(3)$ \\
\hline C15-C19 & $1.442(4)$ \\
\hline C16-C17 & $1.473(4)$ \\
\hline $\mathrm{C} 17-\mathrm{C} 18$ & $1.370(4)$ \\
\hline C18-C19 & $1.461(4)$ \\
\hline C19-C20 & $1.356(4)$ \\
\hline C20-C21 & $1.423(4)$ \\
\hline $\mathrm{C} 21-\mathrm{C} 22$ & $1.346(4)$ \\
\hline
\end{tabular}


Table S8. Selected bond lengths for $\mathbf{C}_{8}-\mathrm{SS}_{-} \mathbf{C l}^{2}$ (II).

\begin{tabular}{|c|c|}
\hline Bond & Length/Å \\
\hline $\mathrm{S} 1-\mathrm{C} 1$ & $1.723(2)$ \\
\hline S1-C5 & $1.729(2)$ \\
\hline S2-C18 & $1.737(2)$ \\
\hline S2-C22 & $1.731(2)$ \\
\hline C11-C1 & $1.726(2)$ \\
\hline $\mathrm{C} 12-\mathrm{C} 22$ & $1.731(2)$ \\
\hline $\mathrm{C} 1-\mathrm{C} 2$ & $1.354(3)$ \\
\hline $\mathrm{C} 2-\mathrm{C} 3$ & $1.424(3)$ \\
\hline $\mathrm{C} 3-\mathrm{C} 4$ & $1.356(3)$ \\
\hline $\mathrm{C} 4-\mathrm{C} 8$ & $1.442(3)$ \\
\hline $\mathrm{C} 4-\mathrm{C} 5$ & $1.461(3)$ \\
\hline $\mathrm{C} 5-\mathrm{C} 6$ & $1.369(3)$ \\
\hline C6-C7 & $1.475(3)$ \\
\hline C6-C23 & $1.501(3)$ \\
\hline $\mathrm{C} 7-\mathrm{C} 8$ & $1.412(3)$ \\
\hline $\mathrm{C} 7-\mathrm{C} 12$ & $1.425(3)$ \\
\hline $\mathrm{C} 8-\mathrm{C} 9$ & $1.410(3)$ \\
\hline C9-C10 & $1.369(3)$ \\
\hline C10-C11 & $1.429(3)$ \\
\hline $\mathrm{C} 11-\mathrm{C} 16$ & $1.430(3)$ \\
\hline $\mathrm{C} 11-\mathrm{C} 12$ & $1.448(3)$ \\
\hline C12-C13 & $1.425(3)$ \\
\hline C13-C14 & $1.367(3)$ \\
\hline C14-C15 & $1.405(3)$ \\
\hline $\mathrm{C} 15-\mathrm{C} 16$ & $1.406(3)$ \\
\hline C15-C19 & $1.446(3)$ \\
\hline C16-C17 & $1.477(3)$ \\
\hline C17-C18 & $1.365(3)$ \\
\hline C18-C19 & $1.460(3)$ \\
\hline C19-C20 & $1.356(3)$ \\
\hline $\mathrm{C} 20-\mathrm{C} 21$ & $1.429(3)$ \\
\hline $\mathrm{C} 21-\mathrm{C} 22$ & $1.345(3)$ \\
\hline
\end{tabular}


Table S9. Selected bond lengths for $\mathbf{C}_{8}-\mathrm{SS}_{-}-\mathbf{C l}^{3}$ (I).

\begin{tabular}{cc}
\hline Bond & Length/A \\
\hline S1-C1 & $1.713(3)$ \\
S1-C11 & $1.734(3)$ \\
C11-C2 & $1.742(3)$ \\
C1-C2 & $1.352(4)$ \\
C2-C3 & $1.435(4)$ \\
C3-C4 & $1.345(3)$ \\
C4-C5 & $1.451(3)$ \\
C5-C9 & $1.402(3)$ \\
C5-C6 & $1.408(3)$ \\
C6-C7 & $1.362(4)$ \\
C7-C8 & $1.427(3)$ \\
C8-C9 & $1.428(3)$ \\
C8-C8 & $1.443(5)$ \\
C9-C10 & $1.477(3)$ \\
C10-C11 & $1.369(3)$ \\
C10-C12 & $1.508(3)$ \\
C12-C13 & $1.533(3)$ \\
C13-C14 & $1.524(3)$ \\
C14-C15 & $1.522(3)$ \\
C15-C16 & $1.522(3)$ \\
C16-C17 & $1.518(3)$ \\
C17-C18 & $1.524(4)$ \\
C18-C19 & $1.527(4)$ \\
\hline &
\end{tabular}


Table S10. Selected bond lengths for $\mathrm{C}_{8}-\mathrm{SS}_{-} \mathbf{C l}^{3}$ (II).

\begin{tabular}{|c|c|}
\hline Bond & Length/Å \\
\hline $\mathrm{S} 1-\mathrm{C} 1$ & $1.709(3)$ \\
\hline S1-C5 & $1.737(3)$ \\
\hline C11-C2 & $1.732(3)$ \\
\hline $\mathrm{C} 1-\mathrm{C} 2$ & $1.344(4)$ \\
\hline $\mathrm{C} 2-\mathrm{C} 3$ & $1.429(4)$ \\
\hline $\mathrm{C} 3-\mathrm{C} 4$ & $1.349(4)$ \\
\hline $\mathrm{C} 4-\mathrm{C} 5$ & $1.464(3)$ \\
\hline $\mathrm{C} 4-\mathrm{C} 8$ & $1.444(3)$ \\
\hline $\mathrm{C} 5-\mathrm{C} 6$ & $1.358(3)$ \\
\hline C6-C7 & $1.474(3)$ \\
\hline C6-C12 & $1.504(3)$ \\
\hline $\mathrm{C} 7-\mathrm{C} 8$ & $1.403(3)$ \\
\hline C8-C9 & $1.401(3)$ \\
\hline C9-C10 & $1.358(4)$ \\
\hline C7-C11 & $1.431(3)$ \\
\hline C10-C11 & $1.425(3)$ \\
\hline C11-C11 & $1.441(4)$ \\
\hline C12-C13 & $1.531(3)$ \\
\hline C13-C14 & $1.525(3)$ \\
\hline C14-C15 & $1.519(3)$ \\
\hline C15-C16 & $1.525(3)$ \\
\hline C16-C17 & $1.517(3)$ \\
\hline C17-C18 & $1.521(4)$ \\
\hline C18-C19 & $1.517(4)$ \\
\hline
\end{tabular}


Table S11. Selected bond lengths for $\mathrm{C}_{8}-\mathrm{SS}_{-}-\mathrm{Cl}^{4}$ (I).

\begin{tabular}{cc}
\hline Bond & Length/A \\
\hline C11-C1 & $1.7367(17)$ \\
S1-C1 & $1.7124(19)$ \\
S1-C11 & $1.7313(17)$ \\
C1-C2 & $1.3440(30)$ \\
C2-C3 & $1.4280(20)$ \\
C3-C4 & $1.3610(20)$ \\
C4-C5 & $1.4520(20)$ \\
C5-C6 & $1.4090(20)$ \\
C5-C9 & $1.4100(20)$ \\
C6-C7 & $1.3650(20)$ \\
C7-C8 & $1.4230(20)$ \\
C8-C9 & $1.4330(20)$ \\
C8-C8 & $1.4370(30)$ \\
C9-C10 & $1.4650(20)$ \\
C10-C11 & $1.3650(20)$ \\
C10-C12 & $1.5060(20)$ \\
C12-C13 & $1.5300(20)$ \\
C13-C14 & $1.5220(20)$ \\
C14-C15 & $1.5210(20)$ \\
C15-C16 & $1.5290(20)$ \\
C16-C17 & $1.5240(20)$ \\
C17-C18 $18-C 19$ & $1.5170(20)$ \\
C18) & $1.5220(30)$ \\
\hline &
\end{tabular}


Table S12. Selected bond lengths for $\mathrm{C}_{8}-\mathrm{SS}_{-} \mathrm{Cl}^{4}$ (II).

\begin{tabular}{cc}
\hline Bond & Length/A \\
\hline C11-C3 & $1.7418(19)$ \\
S1-C1 & $1.7090(2)$ \\
S1-C5 & $1.7337(17)$ \\
C1-C2 & $1.3440(30)$ \\
C2-C3 & $1.4340(20)$ \\
C3-C4 & $1.3570(20)$ \\
C4-C5 & $1.4660(20)$ \\
C4-C8 & $1.4490(20)$ \\
C5-C6 & $1.3630(20)$ \\
C6-C7 & $1.4690(20)$ \\
C6-C12 & $1.5030(20)$ \\
C7-C8 & $1.4110(20)$ \\
C8-C9 & $1.4050(20)$ \\
C7-C11 & $1.4310(20)$ \\
C9-C10 & $1.3640(20)$ \\
C10-C11 & $1.4230(20)$ \\
C11-C11 & $1.4400(30)$ \\
C12-C13 & $1.5290(20)$ \\
C13-C14 & $1.5190(20)$ \\
C14-C15 & $1.5230(20)$ \\
C15-C16 & $1.5190(20)$ \\
C16-C17 & $1.5210(30)$ \\
C17-C18 & $1.5220(30)$ \\
C18-C19 & $1.5190(30)$ \\
\hline &
\end{tabular}


Figure S1. ORTEP diagram for the crystal structure of $\mathbf{C}_{4}-\mathbf{S S}$ with an ellipsoid contour probability level of $50 \%$.

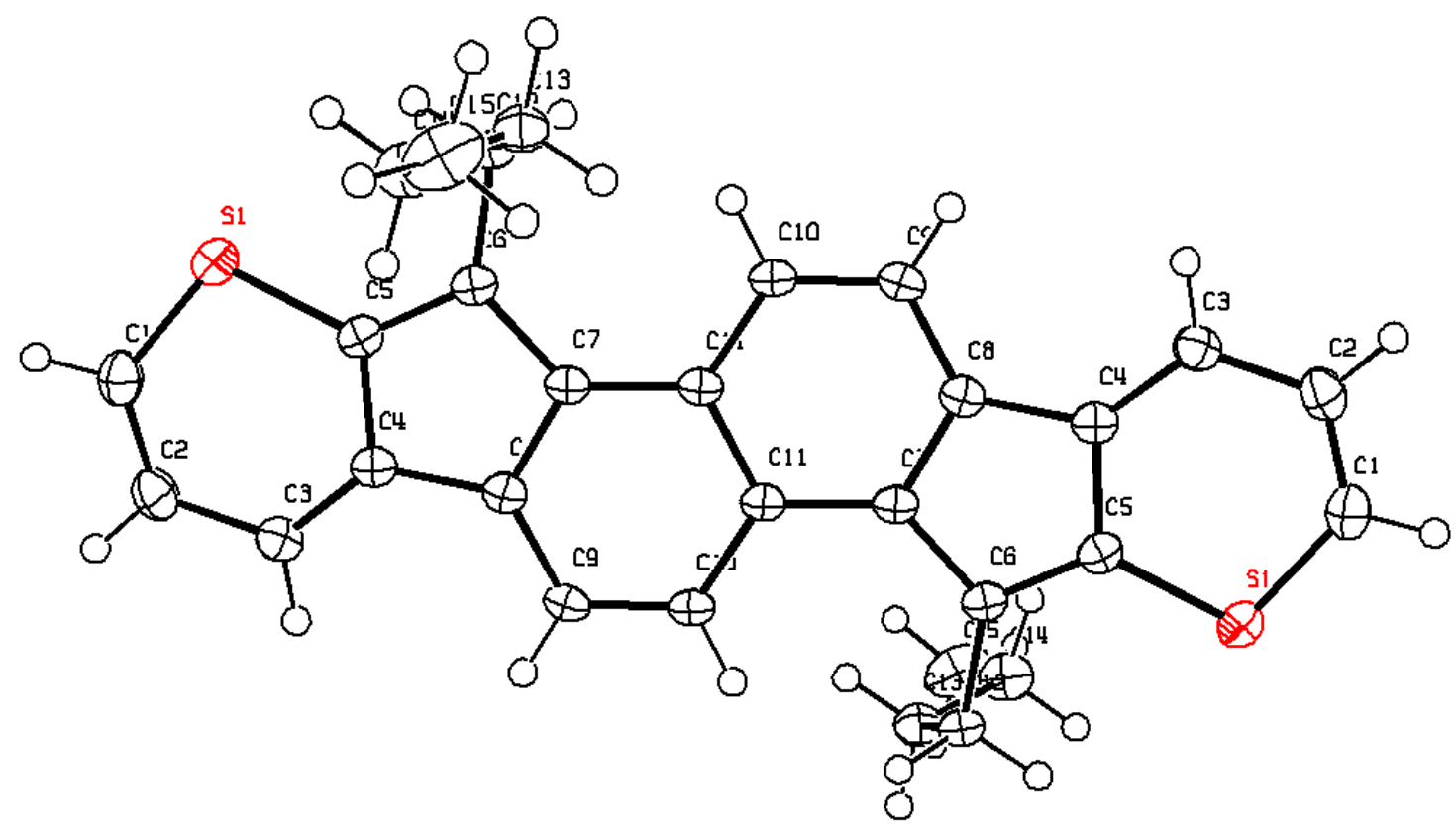


Figure S2. ORTEP diagram for the crystal structure of $\mathbf{C}_{6}-\mathbf{S S}$ with an ellipsoid contour probability level of $50 \%$.

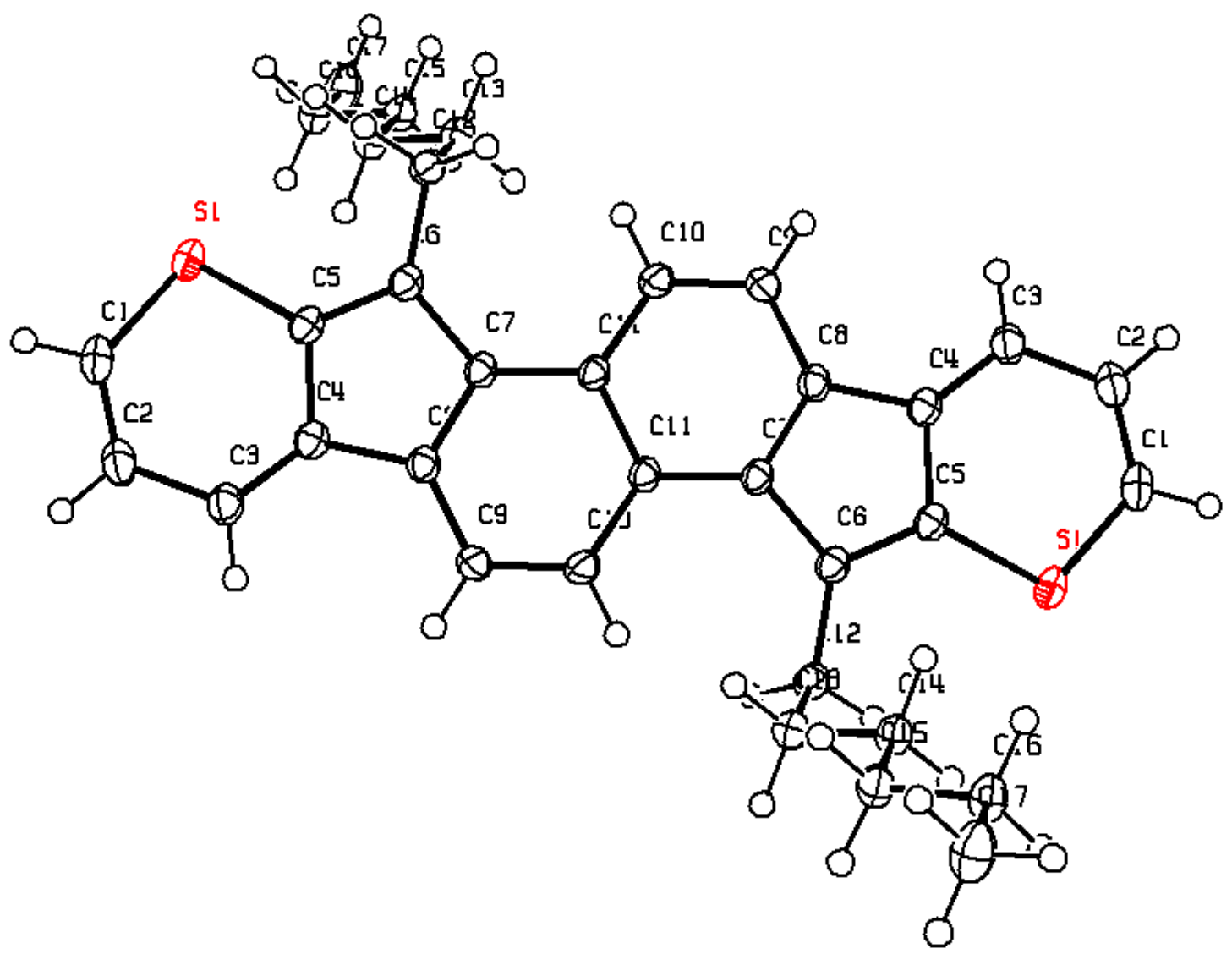


Figure S3. ORTEP diagram for the crystal structure of $\mathbf{C}_{8}-\mathbf{S S}$ with an ellipsoid contour probability level of $50 \%$.

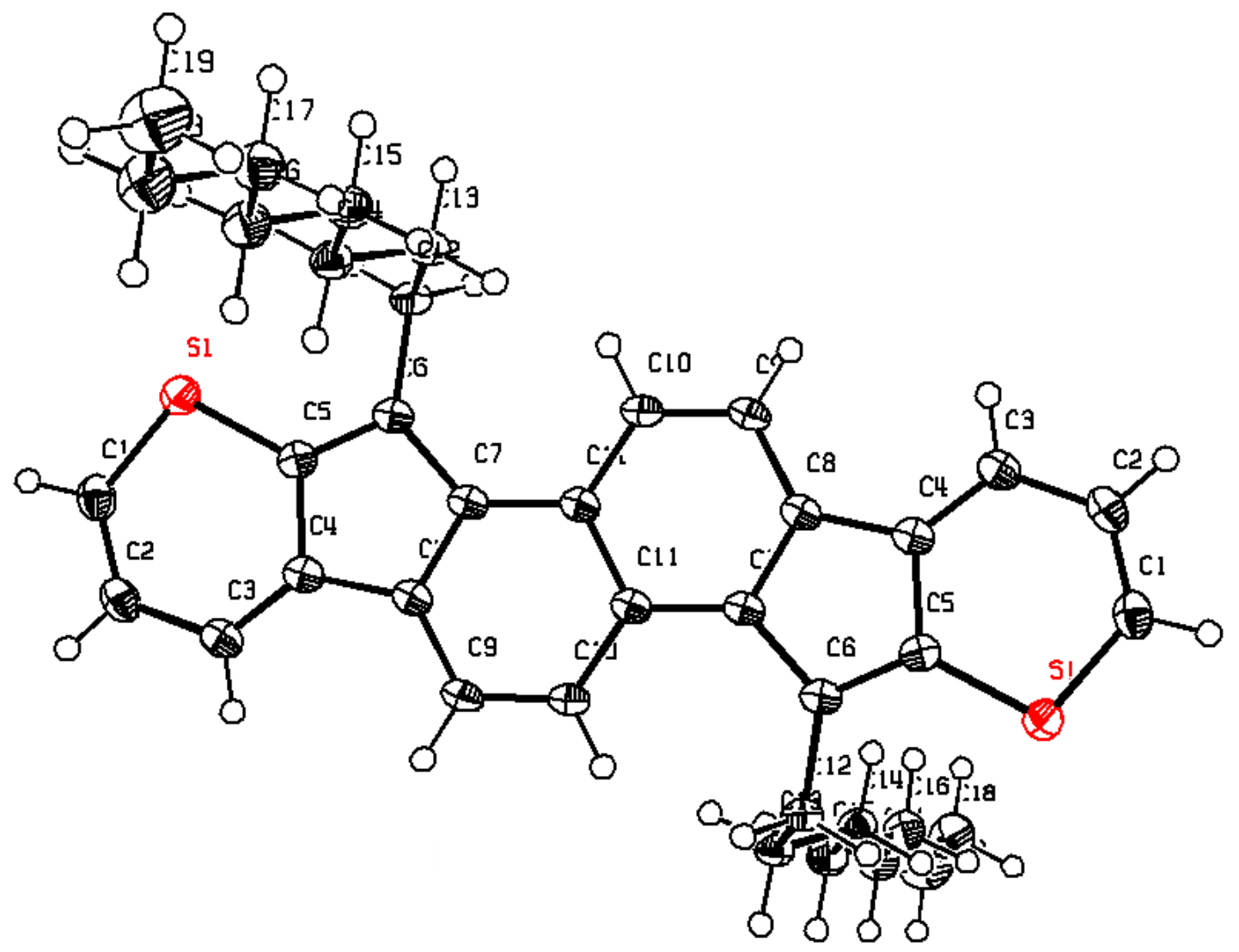


Figure S4. ORTEP diagram for the crystal structure of $\mathbf{C}_{\mathbf{1 0}}-\mathbf{S S}$ with an ellipsoid contour probability level of $50 \%$.

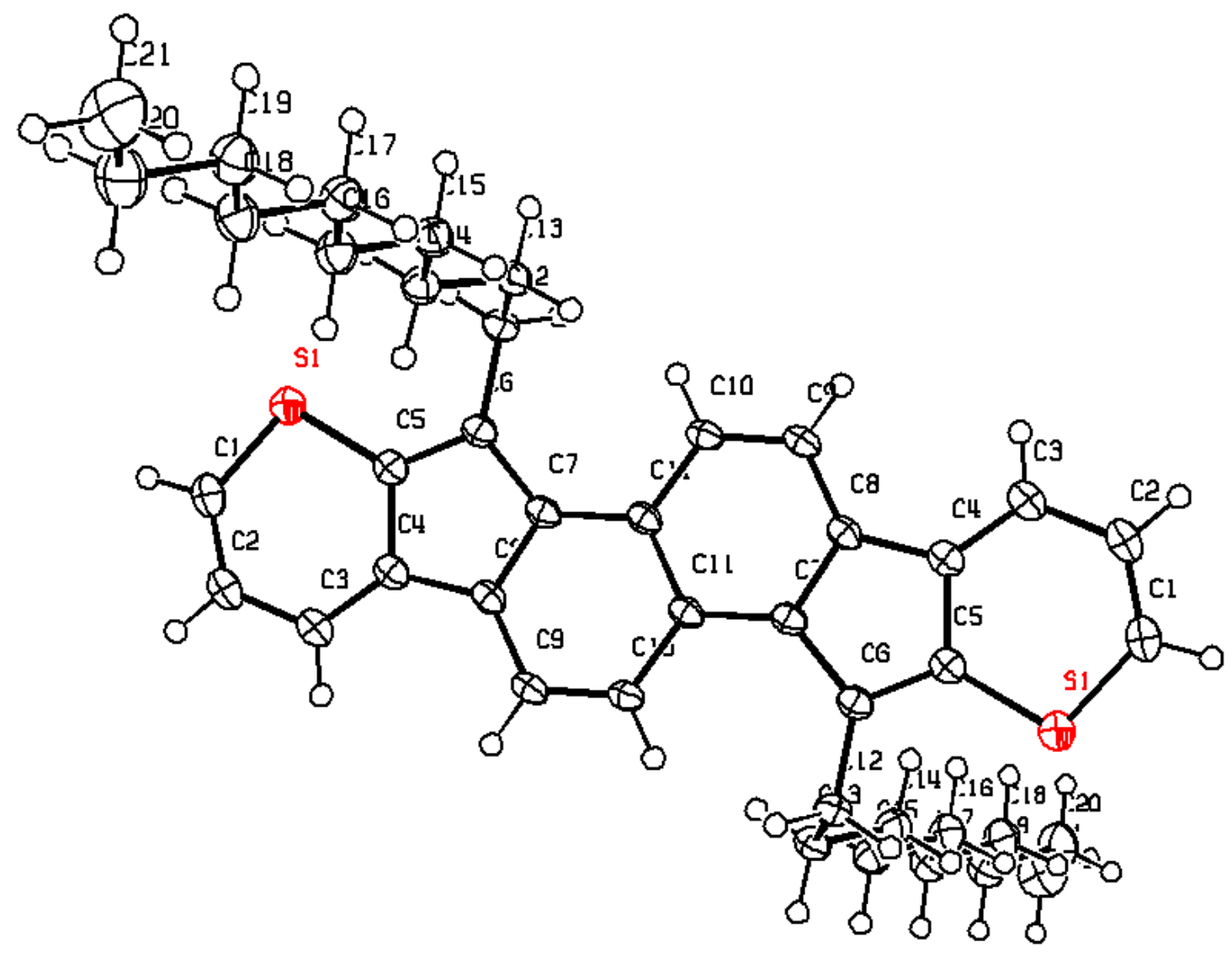


Figure S5. ORTEP diagram for the crystal structure of $\mathbf{C}_{8}-\mathbf{S S}-\mathbf{C l}^{2}$ (I) with an ellipsoid contour probability level of $50 \%$.




Figure S6. ORTEP diagram for the crystal structure of $\mathbf{C}_{8}-\mathbf{S S}-\mathbf{C l}^{\mathbf{2}}$ (II) with an ellipsoid contour probability level of 50\%.

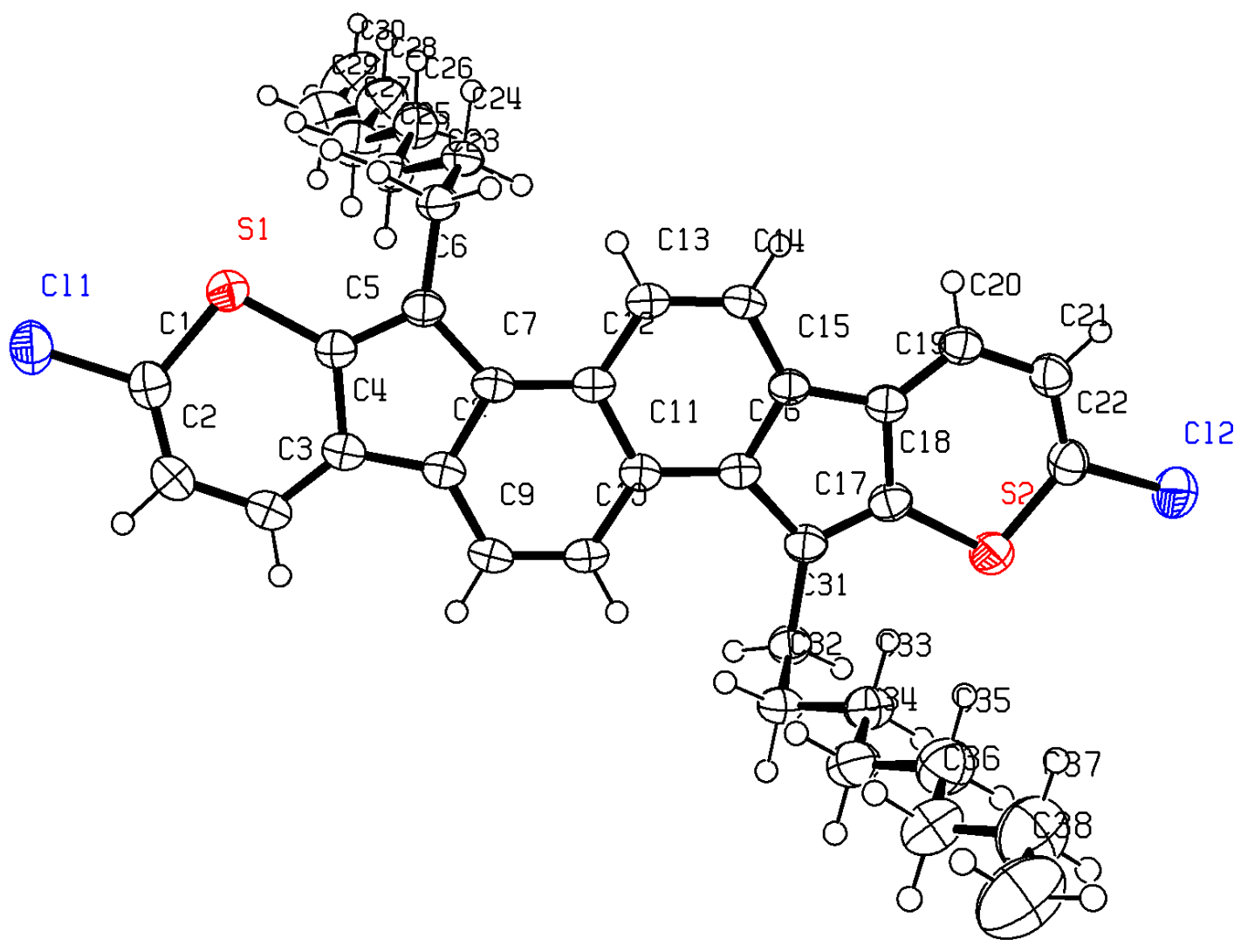


Figure S7. ORTEP diagram for the crystal structure of $\mathbf{C}_{8}-\mathbf{S S}-\mathbf{C l}^{3}$ (I) with an ellipsoid contour probability level of $50 \%$.

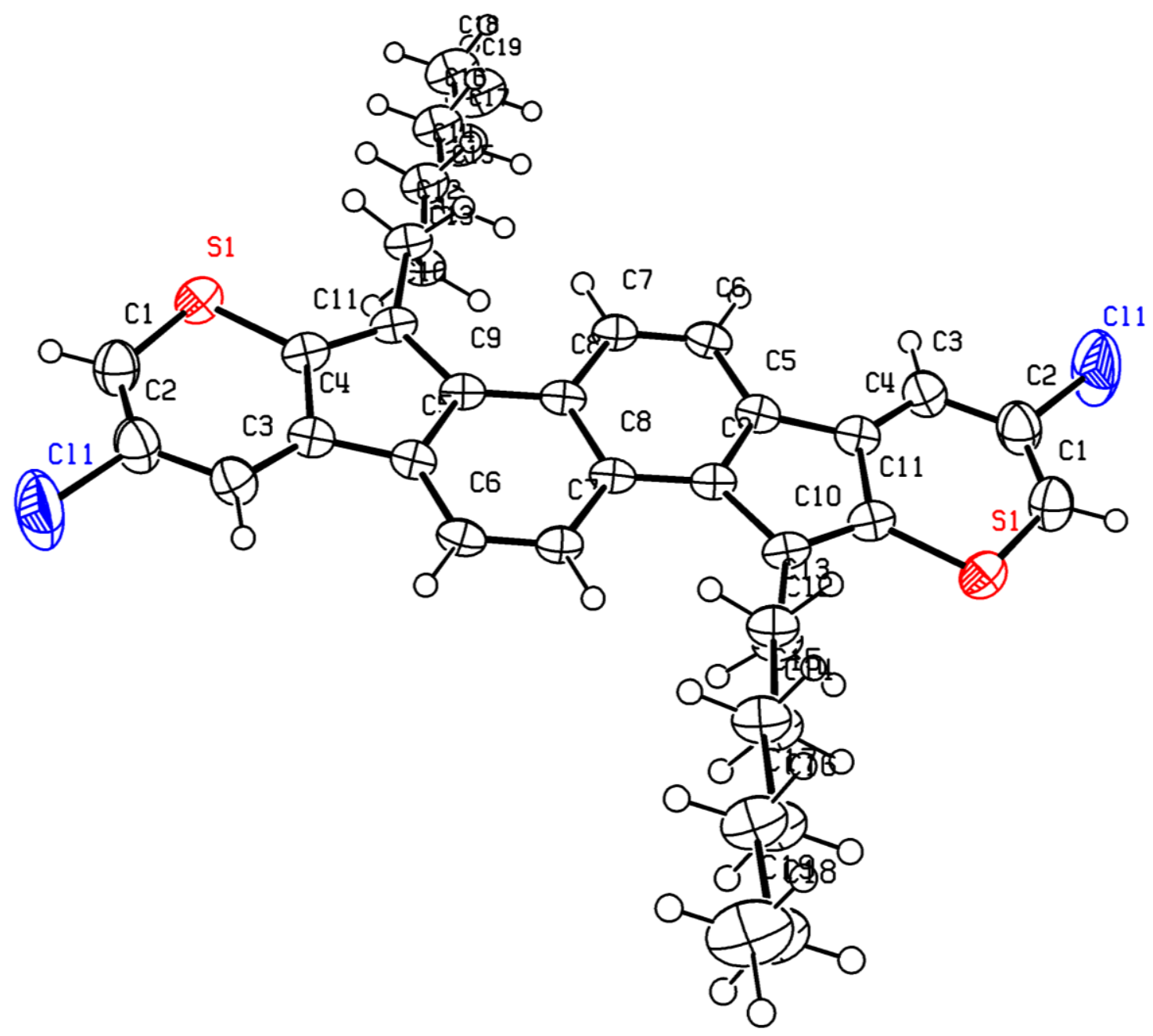


Figure S8. ORTEP diagram for the crystal structure of $\mathbf{C}_{8}-\mathbf{S S}-\mathbf{C l}^{\mathbf{3}}$ (II) with an ellipsoid contour probability level of $50 \%$.

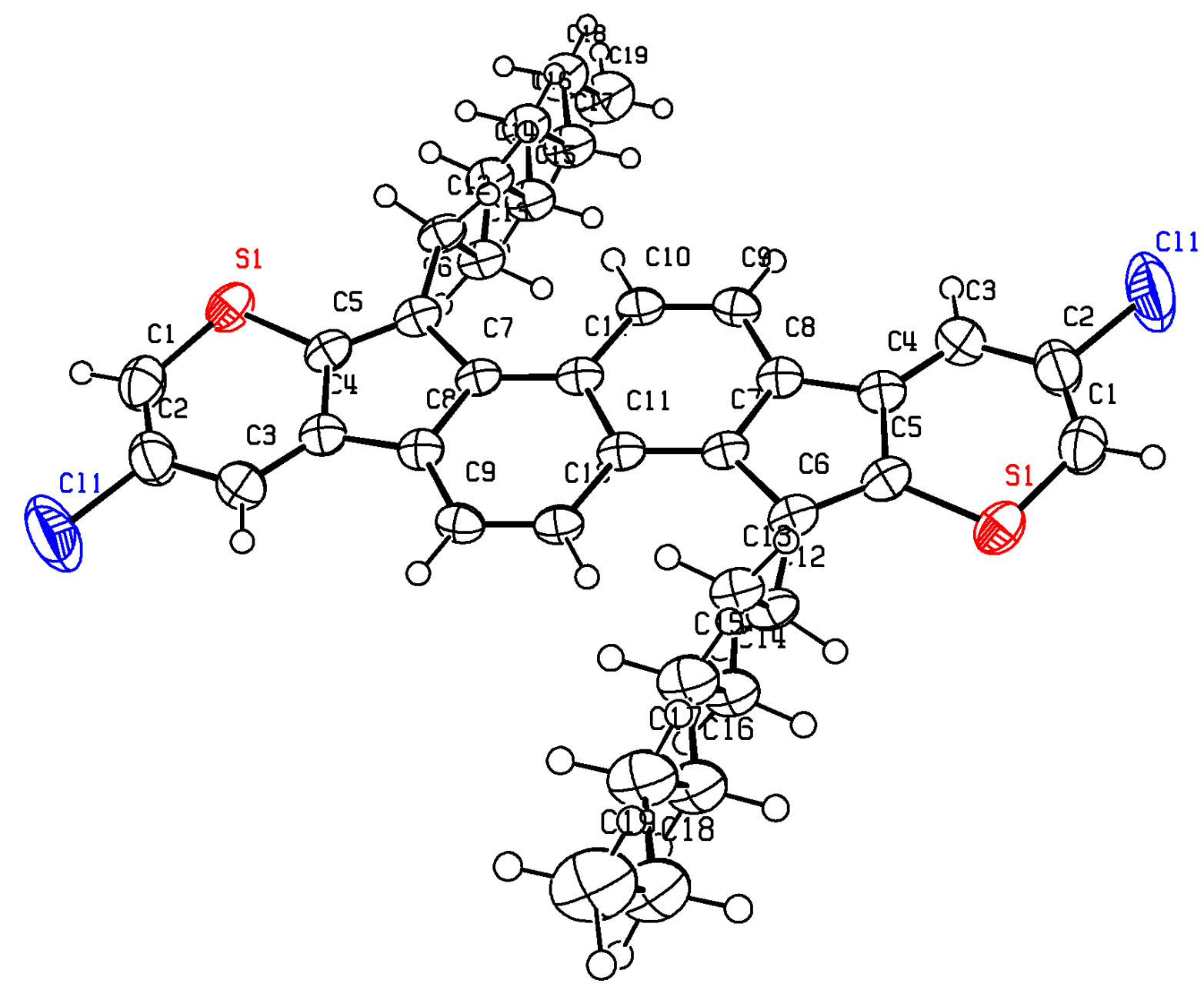


Figure S9. ORTEP diagram for the crystal structure of $\mathbf{C}_{8}-\mathbf{S S}_{-} \mathbf{C l}^{4}$ (I) with an ellipsoid contour probability level of $50 \%$.

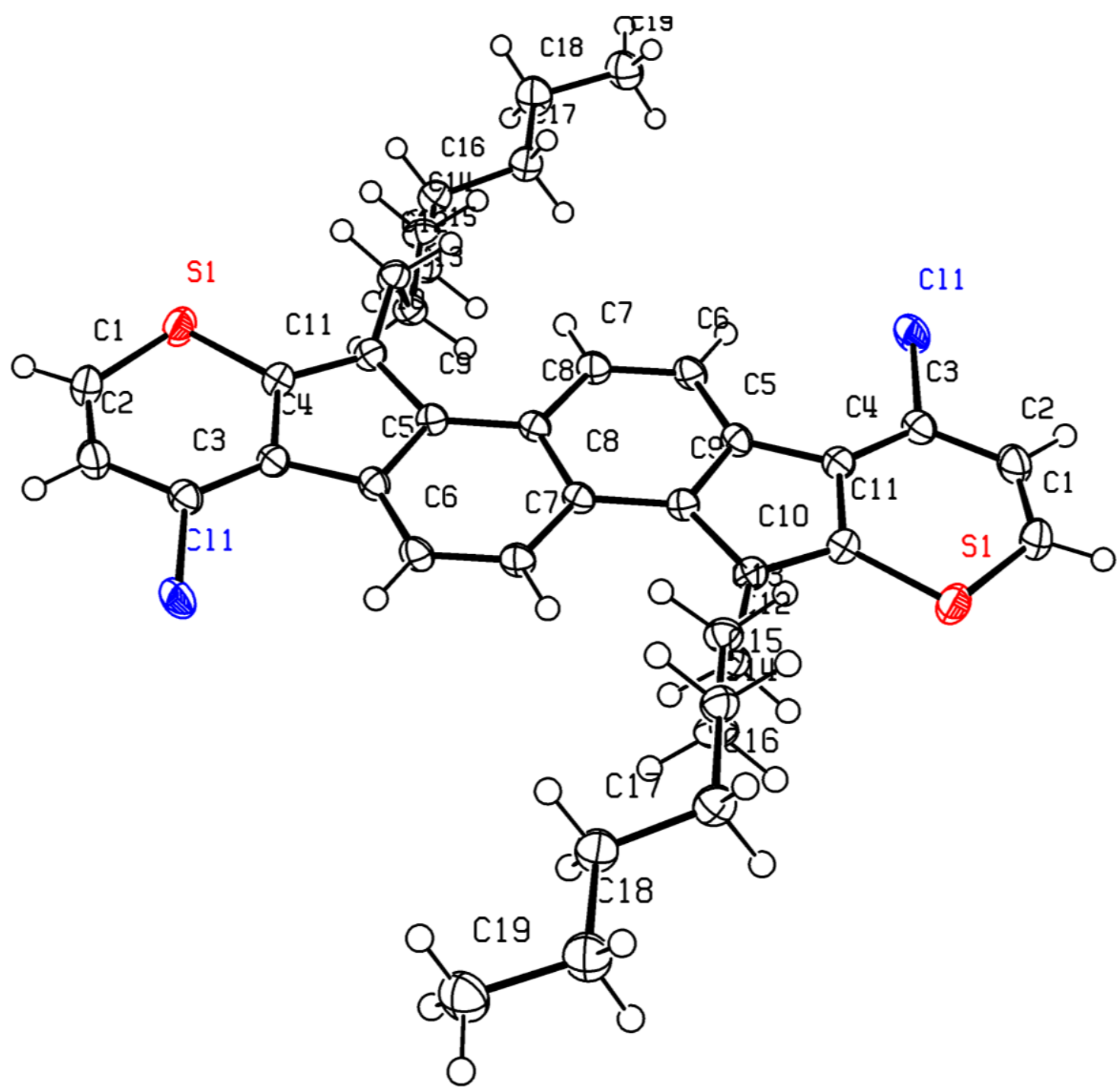


Figure S10. ORTEP diagram for the crystal structure of $\mathbf{C}_{8}-\mathbf{S S}-\mathbf{C l}^{4}$ (II) with an ellipsoid contour probability level of 50\%.

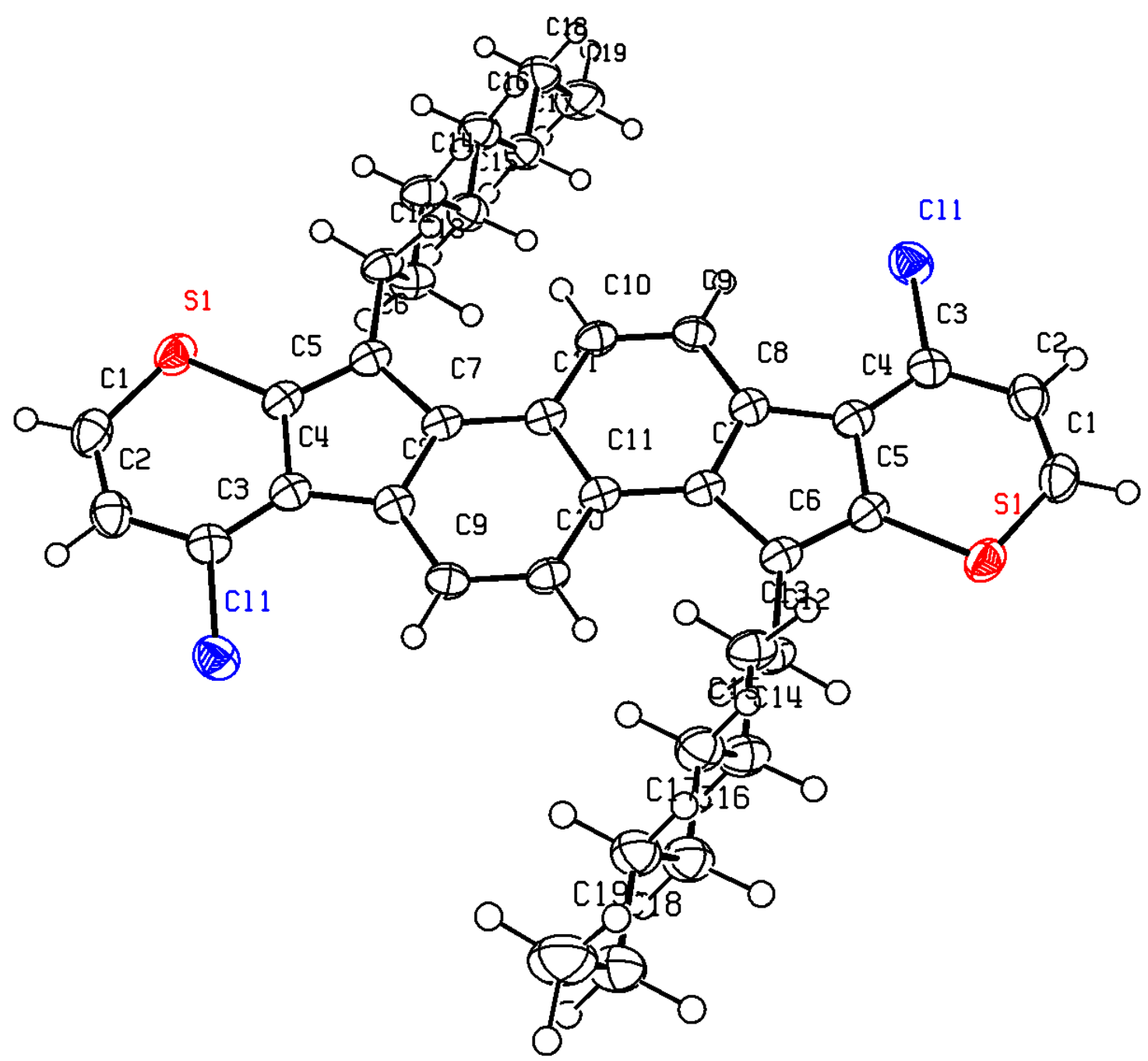


Figure S11. Crystal packing structures of $\mathbf{C}_{8}-\mathbf{S S}-\mathbf{C l}^{2}$ grown from different solvents.

(a)

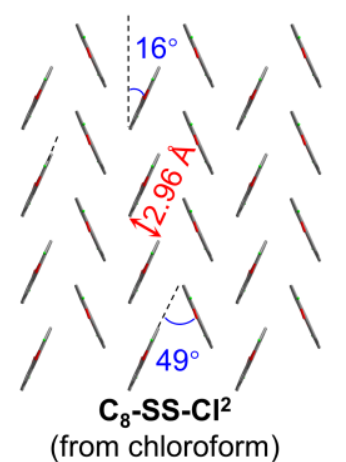

(c)

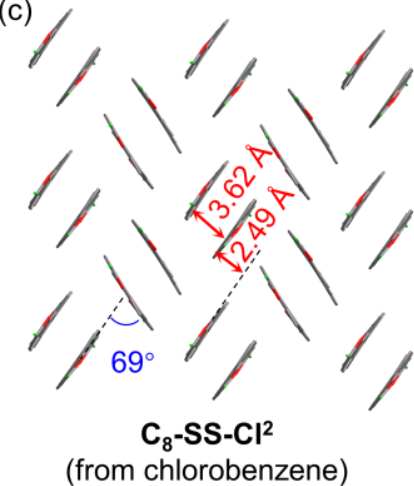

(b)

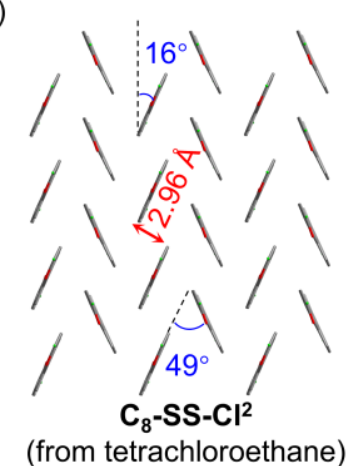

(d)

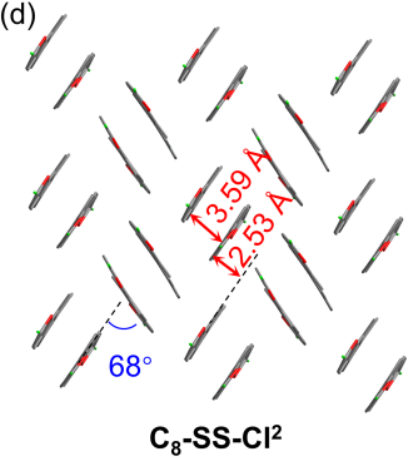

(from o-dichlorobenzene) 


\section{Theoretical calculations}

All the theoretical calculations were conducted with the Gaussian 16 program. ${ }^{\mathrm{S}}$ The calculations of the molecular frontier orbitals and the nucleus-independent chemical shift were conducted using the B3LYP method and 6-311G(d,p) basis set. The transfer integrals between the HOMOs of adjacent molecules were calculated based on the crystal geometries using PW91 exchange and PW91 correlation functionals with the $6-31 \mathrm{G}^{*}$ basis set. ${ }^{\mathrm{S} 4, \mathrm{~S} 5}$

Figure S12. Molecular structures and calculated frontier orbitals of $\mathbf{C}_{4}-\mathbf{S S}, \mathbf{C}_{6}-\mathbf{S S}, \mathbf{C}_{8}-\mathbf{S S}$, and C10-SS.

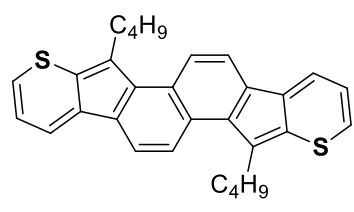

$\mathrm{C}_{4}-\mathrm{SS}$

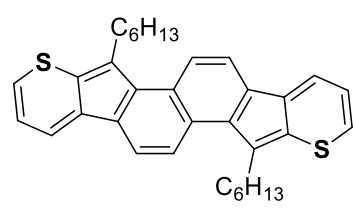

$\mathrm{C}_{6}-\mathrm{SS}$

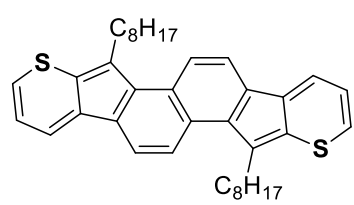

$\mathrm{C}_{8}-\mathrm{SS}$

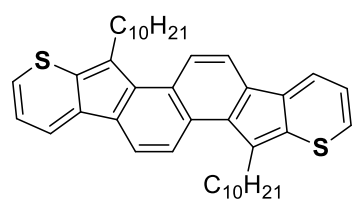

$\mathrm{C}_{10}$-SS

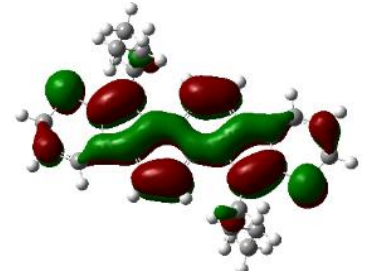

HOMO

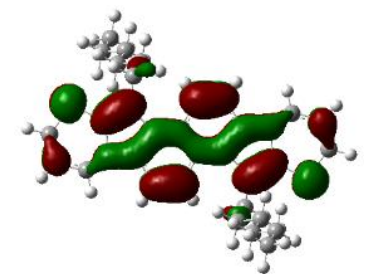

HOMO

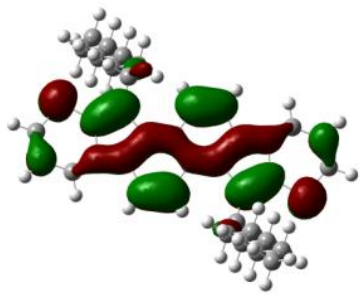

HOMO

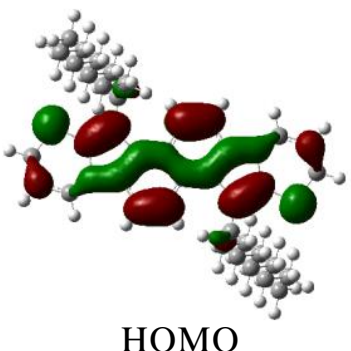

HOMO

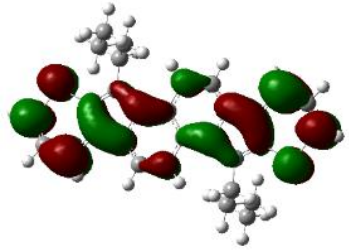

LUMO

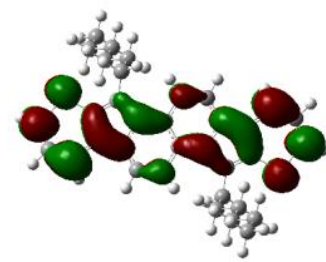

LUMO
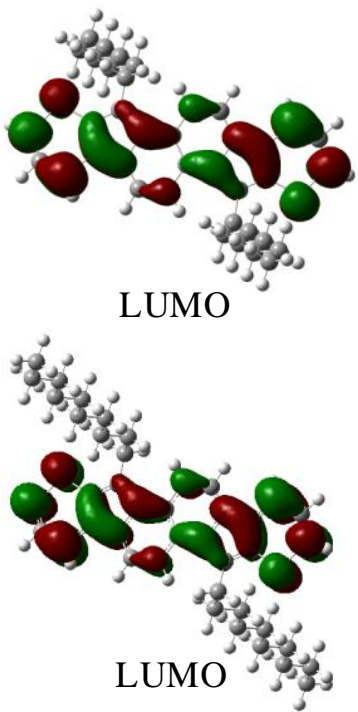


\section{OFET Device Fabrication}

The bottom-gate top-contact (BGTC) devices based on the single-crystals of cyclopenta $[b]$ thiopyran derivatives growing on the OTS modified $\mathrm{SiO}_{2} / \mathrm{Si}$ substrates were fabricated with the "gold strips" method to investigate charge transport properties. The $\mathrm{SiO}_{2} / \mathrm{Si}$ wafers used to grow crystals were first cleaned with hot piranha solution $\left(\mathrm{H}_{2} \mathrm{SO}_{4} / \mathrm{H}_{2} \mathrm{O}_{2}=2: 1\right)$ followed by a copious rinsing with deionized water. After drying at $70^{\circ} \mathrm{C}$, the octadecyltrichlorosilane surface modification was carried out by the vapor-deposition method. Those cleaned wafers were put into a Petri dish with a small drop of OTS in the center. Then the dish was heated in a vacuum oven at $120^{\circ} \mathrm{C}$ for 3 hours to form an OTS self-assembled monolayer on the surface of the $\mathrm{SiO}_{2} / \mathrm{Si}$ wafers. When the temperature returned to approximately room temperature, the OTS modified wafers were rinsed with hexane, ethanol, and chloroform and finally were blown dry by nitrogen. On the cleaned $\mathrm{SiO}_{2} / \mathrm{Si}$ wafer modified by OTS was put a drop of the solution containing the cyclopenta[ $b]$ thiopyran-based semiconductor $(1 \mathrm{mg} / \mathrm{mL})$. The solvent was controlled to slowly evaporate in several hours. Then the single crystals in micrometer scale may grow on the substrates. Different solvents (toluene, chlorobenzene, chloroform, dimethyl sulfoxide) were used for crystal preparation, and the best quality crystals were selected on a Micromanipulator station coupled with an optical microscope to further fabricate the transistor device. Two pieces of the Au films, approximately $150 \mu \mathrm{m} \times 30 \mu \mathrm{m}$, were glued onto the selected single crystals via the electrostatic forces with the help of the mechanical probes in the Micromanipulator. The above mentioned Au films were prepared as follow: firstly, a patterned Au thin film with a thickness around $100 \mathrm{~nm}$ was predeposited on a Si wafer by thermal evaporation with a copper mask. Then, two small pieces of the Au films with desired sizes were peeled off from the Si substrate with the tip of the mechanical probe and transferred onto the single crystals as source and drain electrodes. The Si substrate functioned as the gate electrode. The current-voltage $(I-V)$ characteristics of the device were measured on the Micromanipulator station using a Keithley 4200 source meter under ambient conditions. 


\section{Characterization of solution-deposited thin films and transistors}

Figure S13. Reflected polarized-light micrographs for (a) $\mathbf{C}_{4}-\mathbf{S S}$, (b) $\mathbf{C}_{6}-\mathbf{S S}$, (c) $\mathrm{C}_{8}-\mathbf{S S}$, (d) $\mathrm{C}_{10-}$


$\mathrm{Cl}^{4}$ (II) on OTS-treated $\mathrm{SiO}_{2} / \mathrm{Si}$.

(a)

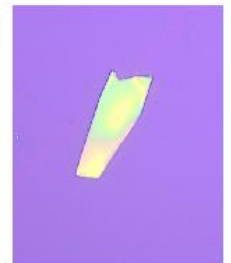

(d)

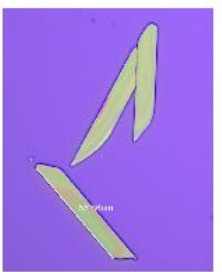

(g)
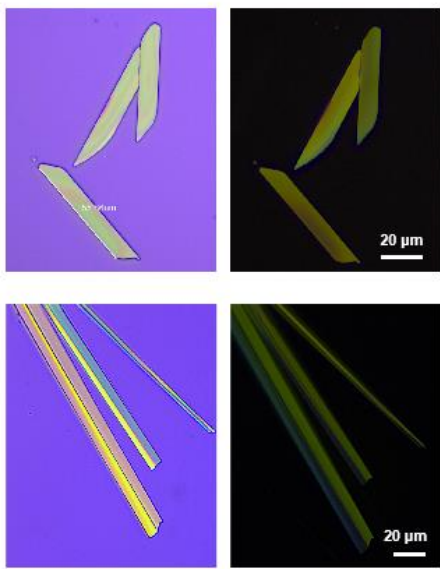

(b)



(e)

e)

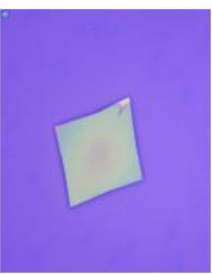

(h)
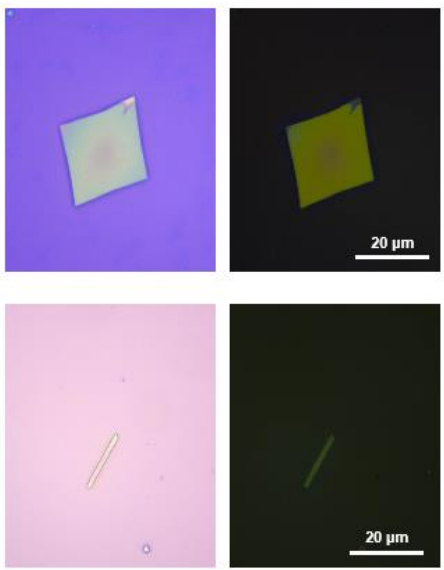

(c)
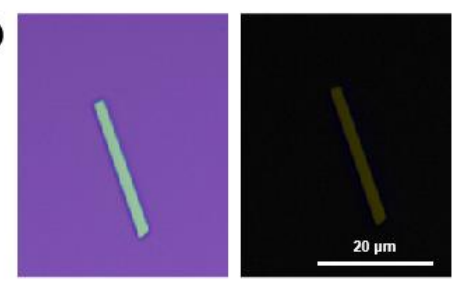

(f)

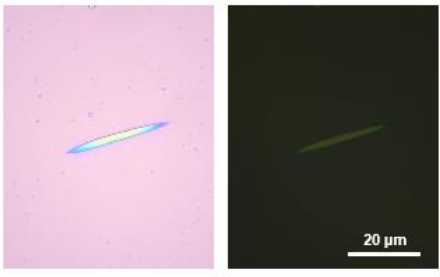

(i)
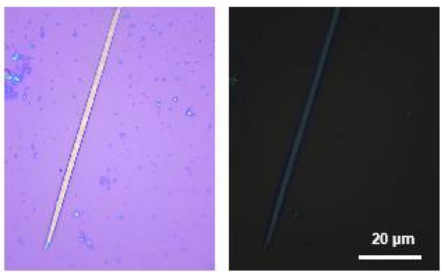
Figure S14. Representative transfer current-voltage (I-V) curves for the single crystal transistors of (a) $\mathbf{C}_{\mathbf{4}}-\mathbf{S S}$, (b) $\mathbf{C}_{6}-\mathbf{S S}$, (c) $\mathbf{C}_{8}-\mathbf{S S}$, (d) $\mathbf{C}_{\mathbf{1 0}}-\mathbf{S S}$, (e) $\mathbf{C}_{8}-\mathbf{S S}_{-} \mathbf{C l}^{2}$ (I), (f) $\mathbf{C}_{8}-\mathbf{S S}_{-} \mathbf{C l}^{\mathbf{3}}$ (I),

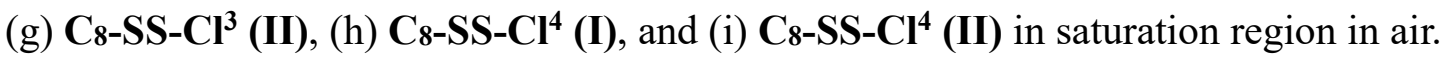

Figure S15. Output curves of (a) $\mathbf{C}_{4}-\mathbf{S S}$, (b) $\mathbf{C}_{6}-\mathbf{S S}$, (c) $\mathbf{C}_{8}-\mathbf{S S}$, (d) $\mathbf{C}_{10}-\mathbf{S S}$, (e) $\mathbf{C}_{8}-\mathbf{S S}_{-} \mathbf{C l}^{2}$ (I), (f) $\mathrm{C}_{8}-\mathrm{SS}_{-} \mathrm{Cl}^{3}$ (I), (g) $\mathrm{C}_{8}-\mathrm{SS}_{-} \mathrm{Cl}^{3}$ (II), (h) $\mathrm{C}_{8}-\mathrm{SS}_{-} \mathrm{Cl}^{4}$ (I), and (i) $\mathrm{C}_{8}-\mathrm{SS}_{-} \mathrm{Cl}^{4}$ (II) as measured in air at room temperature. The output curves were obtained by applying a drain-source voltage from $5 \mathrm{~V}$ to $-60 \mathrm{~V}$ with the gate voltage kept at $0 \mathrm{~V},-10 \mathrm{~V},-20 \mathrm{~V},-30 \mathrm{~V},-40 \mathrm{~V},-50 \mathrm{~V},-60 \mathrm{~V}$, respectively.

Figure S16. Mobilities and transfer current-voltage $(I-V)$ curves for the single crystal transistors based on $\mathbf{C}_{8}-\mathbf{S S}$ and $\mathbf{C}_{8}-\mathbf{S S}-\mathbf{C l}^{2}$ (II) over a period of 15 days.
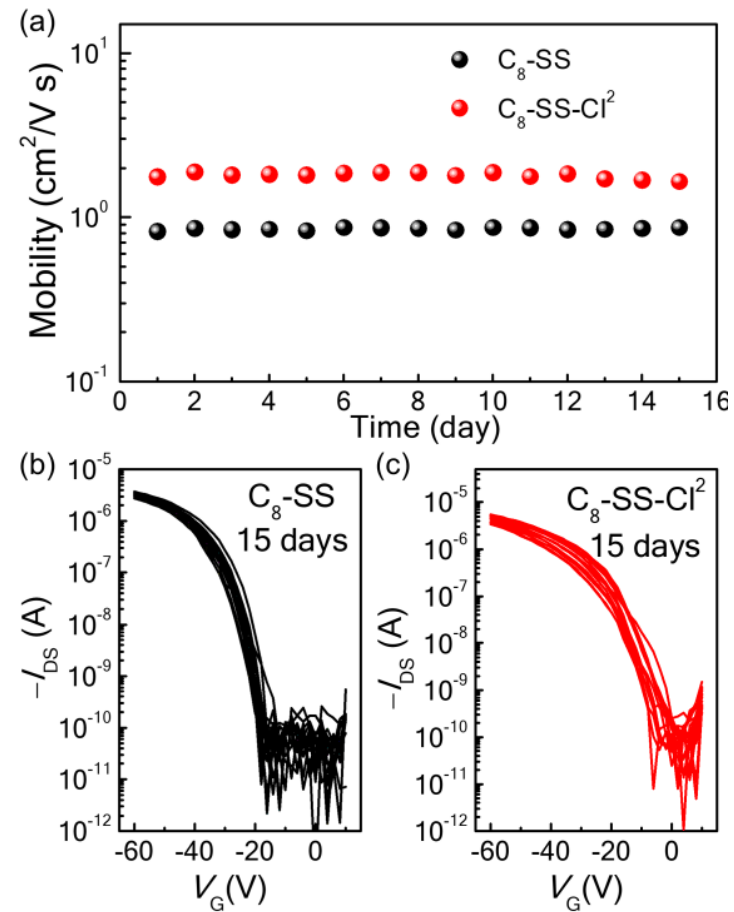


\section{6. ${ }^{1} \mathrm{H}$ and ${ }^{13} \mathrm{C}$ NMR spectra of new compounds}

Figure S17. ${ }^{1} \mathrm{H}$ NMR spectrum of $\mathbf{3 a}$ in $\mathrm{CDCl}_{3}$.
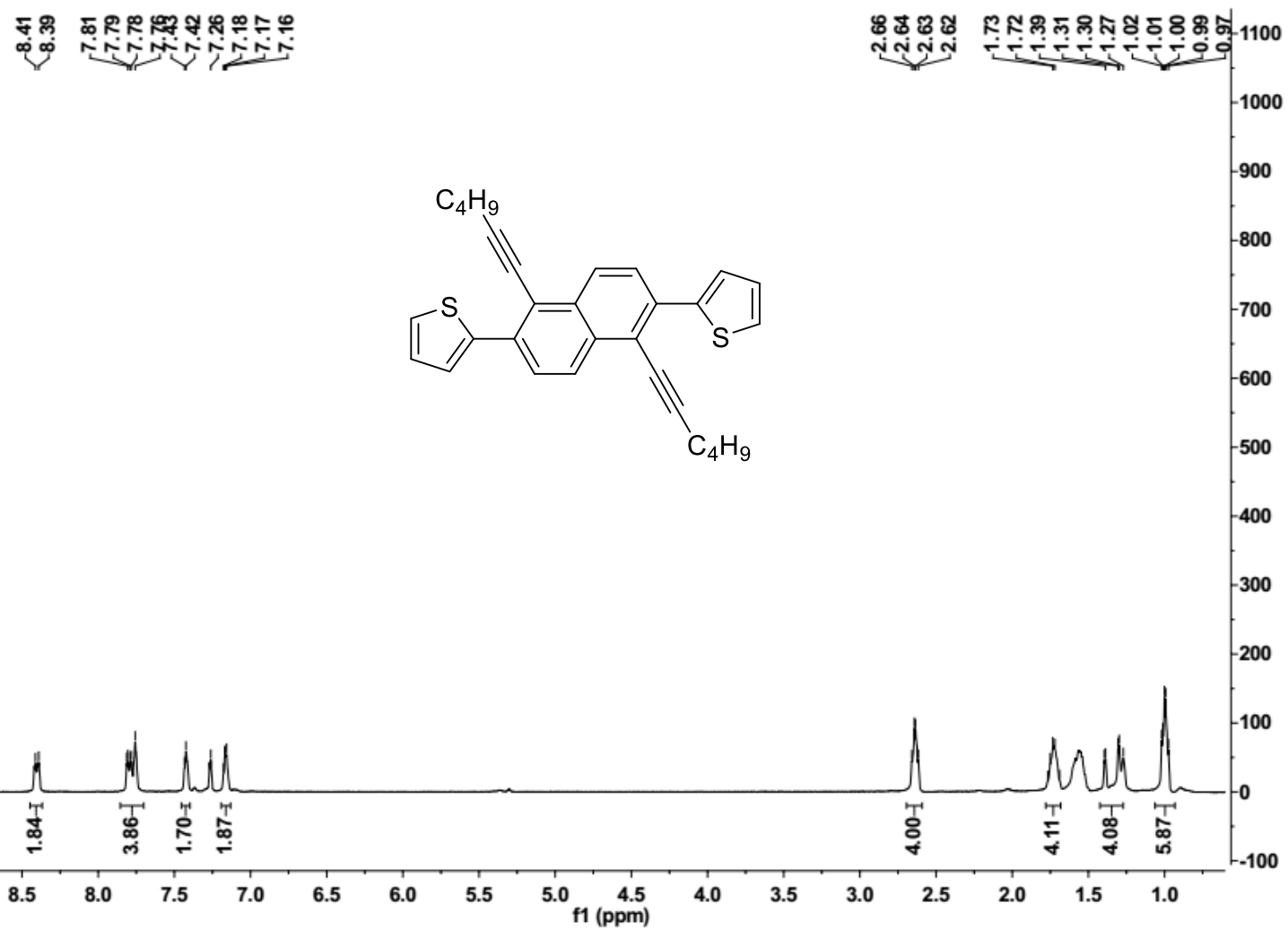

Figure S18. ${ }^{13} \mathrm{C}$ NMR spectrum of $\mathbf{3 a}$ in $\mathrm{CDCl}_{3}$.

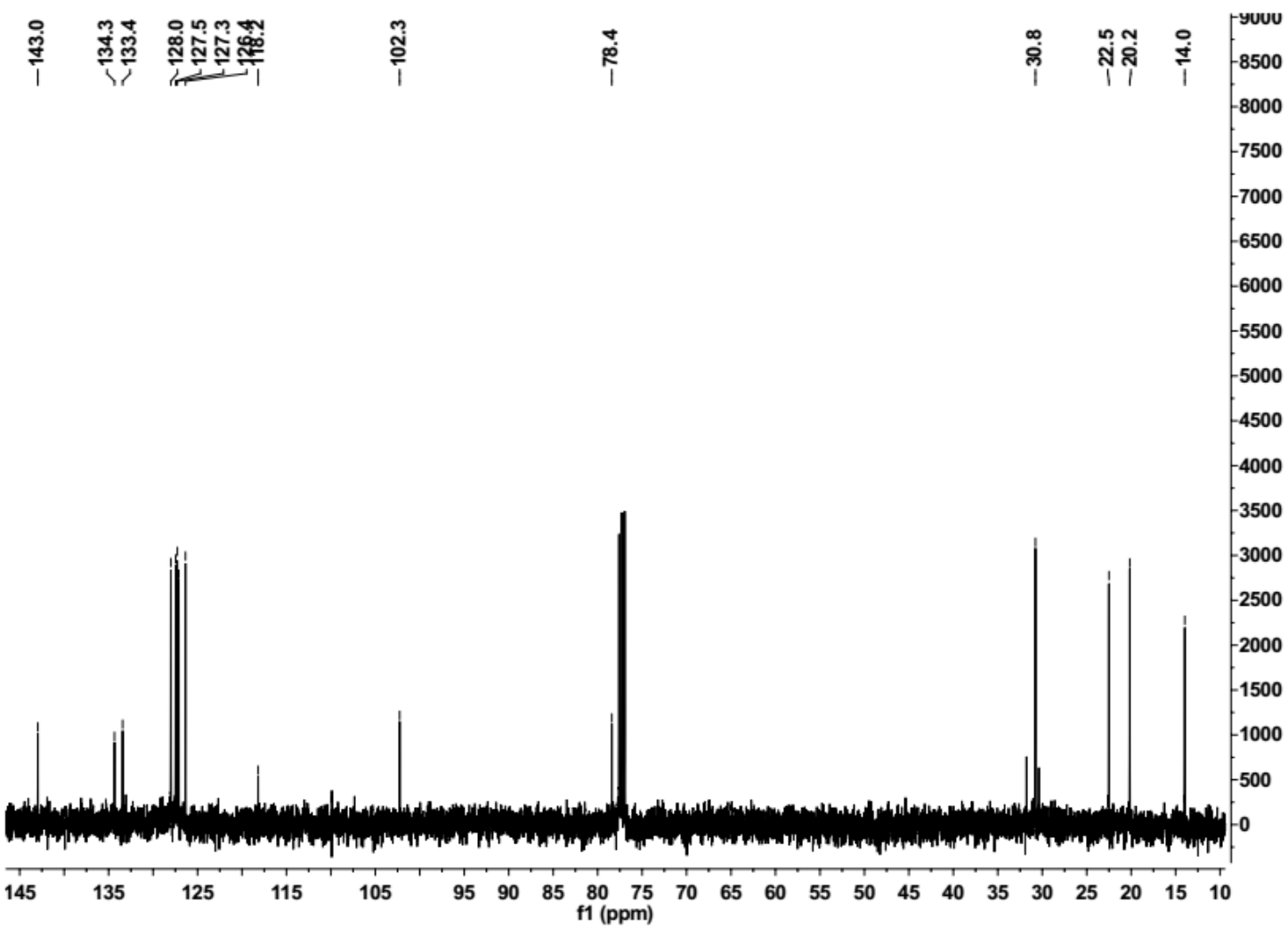


Figure S19. ${ }^{1} \mathrm{H}$ NMR spectrum of $\mathbf{3 b}$ in $\mathrm{CDCl}_{3}$.

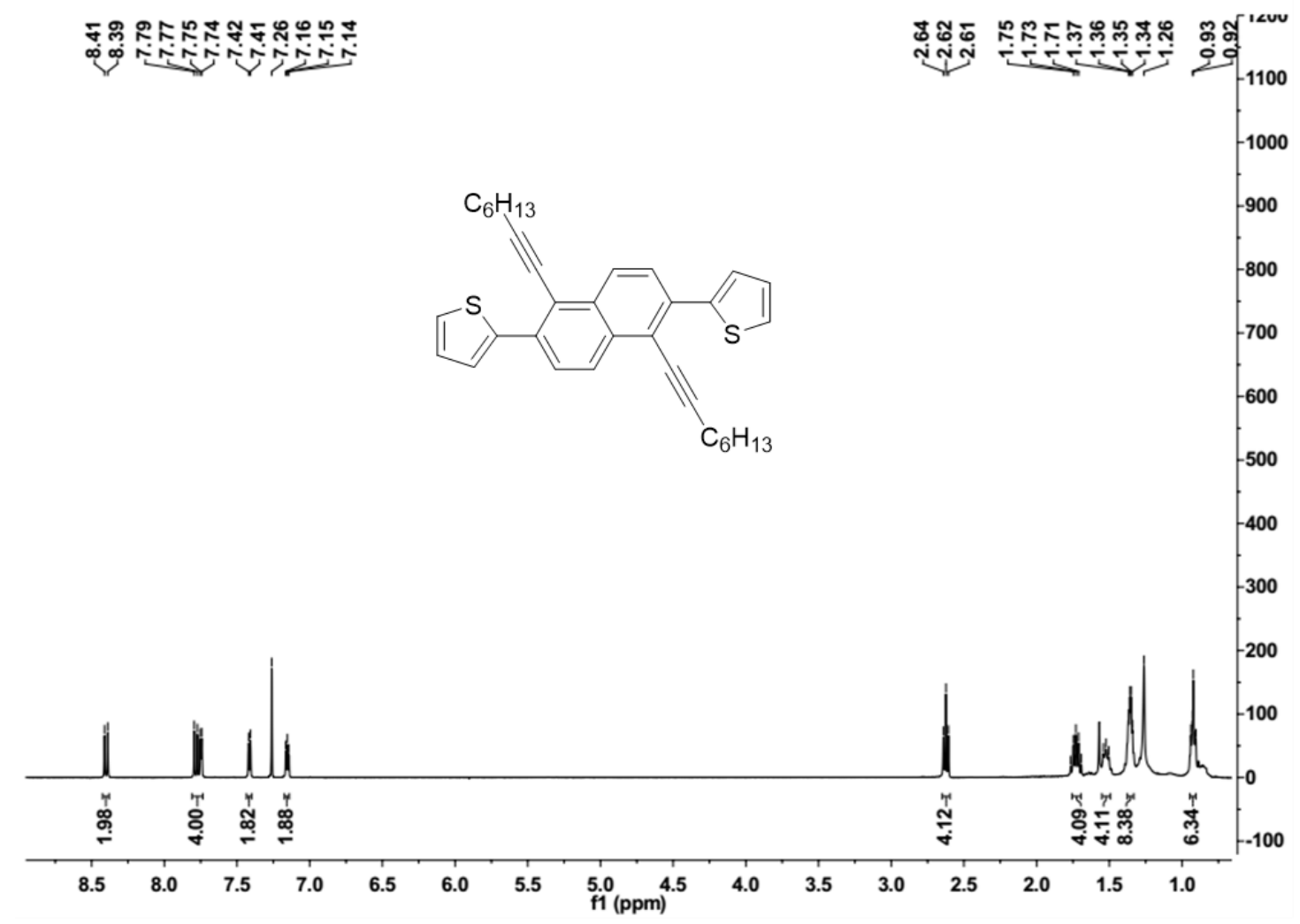

Figure S20. ${ }^{13} \mathrm{C}$ NMR spectrum of $\mathbf{3 b}$ in $\mathrm{CDCl}_{3}$.

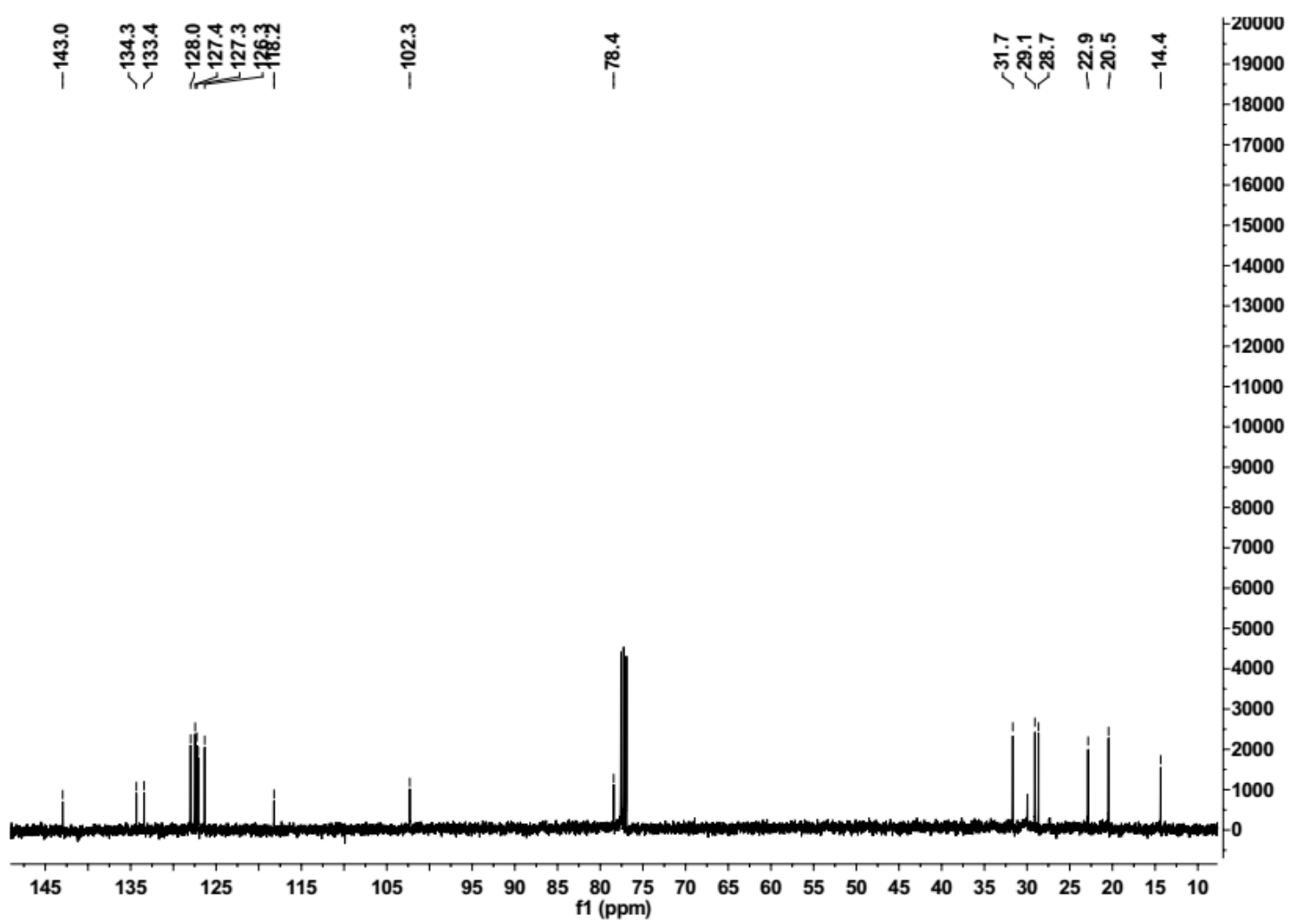


Figure S21. ${ }^{1} \mathrm{H}$ NMR spectrum of $\mathbf{3 c}$ in $\mathrm{CDCl}_{3}$.

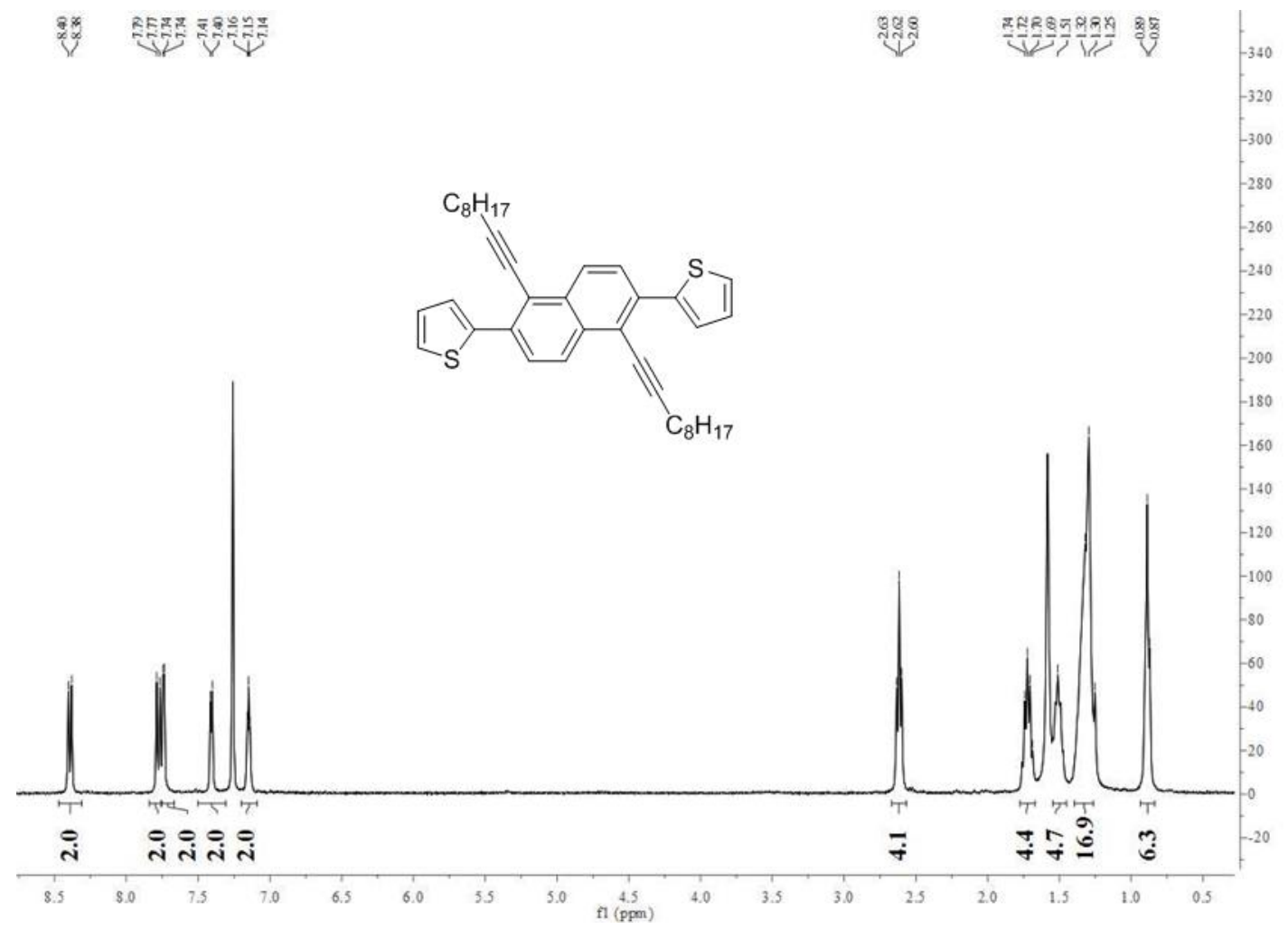

Figure S22. ${ }^{13} \mathrm{C}$ NMR spectrum of $\mathbf{3 c}$ in $\mathrm{CDCl}_{3}$.

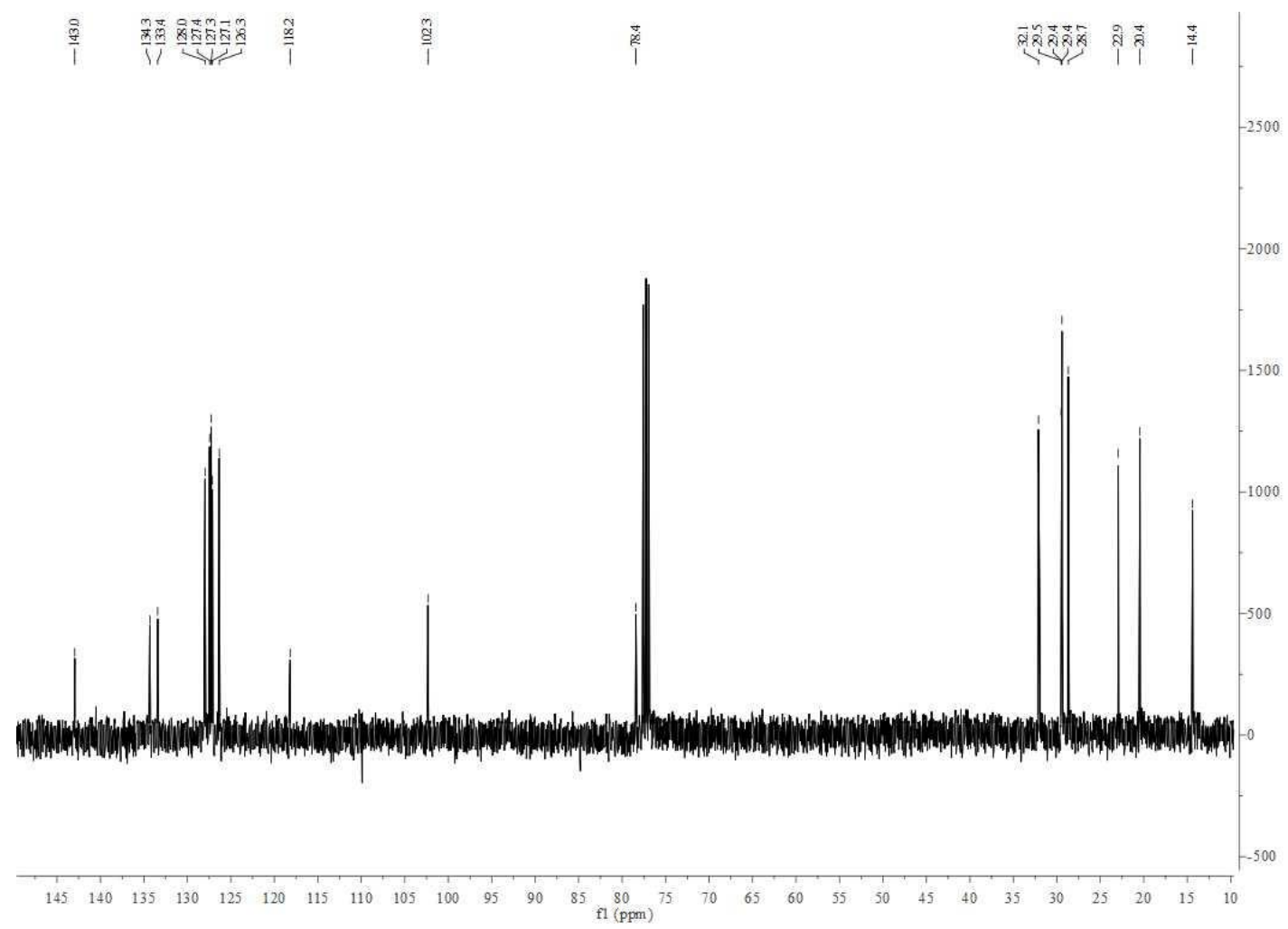


Figure S23. ${ }^{1} \mathrm{H}$ NMR spectrum of $\mathbf{3 d}$ in $\mathrm{CDCl}_{3}$.

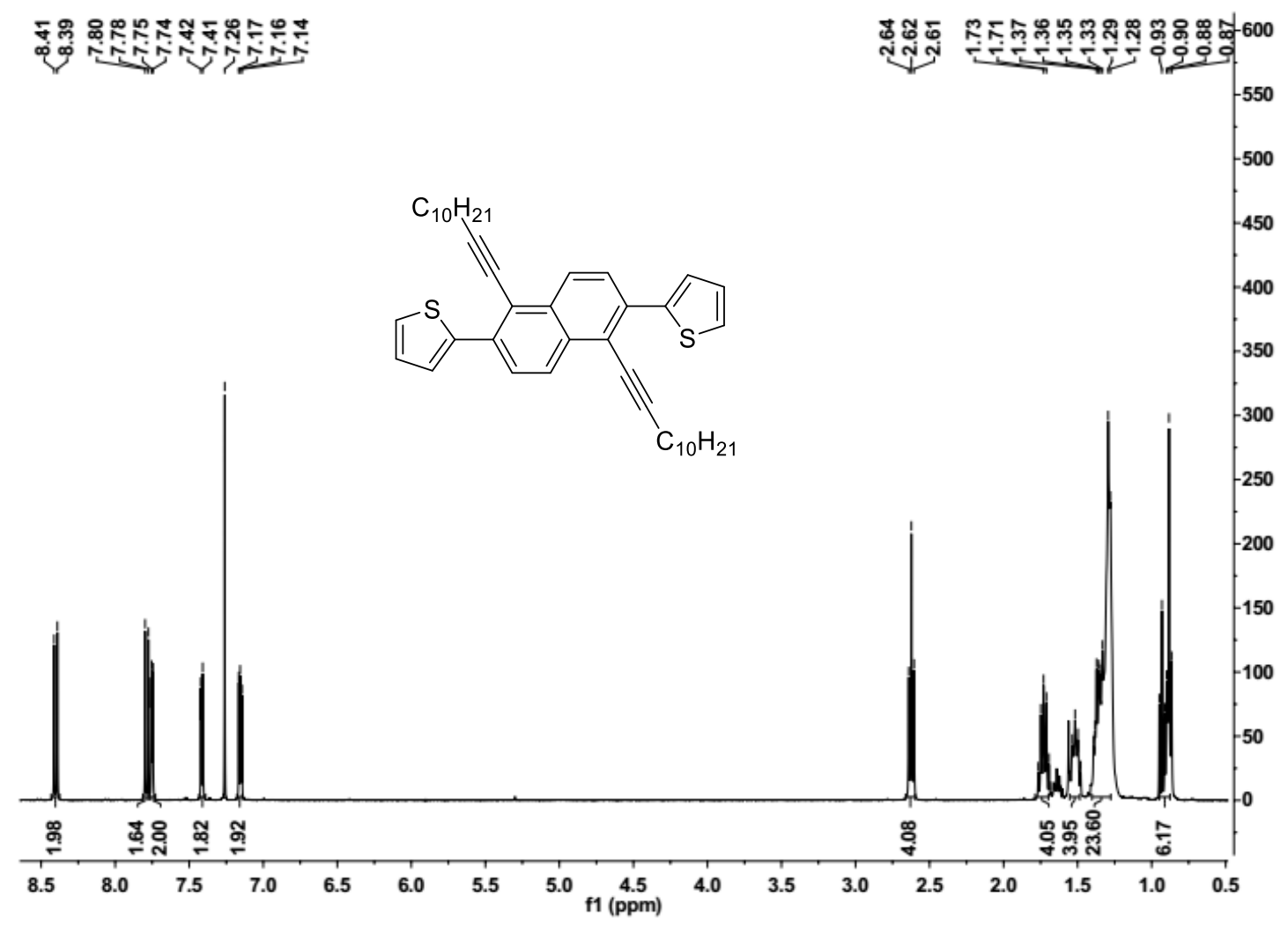

Figure S24. ${ }^{13} \mathrm{C}$ NMR spectrum of $\mathbf{3 d}$ in $\mathrm{CDCl}_{3}$.

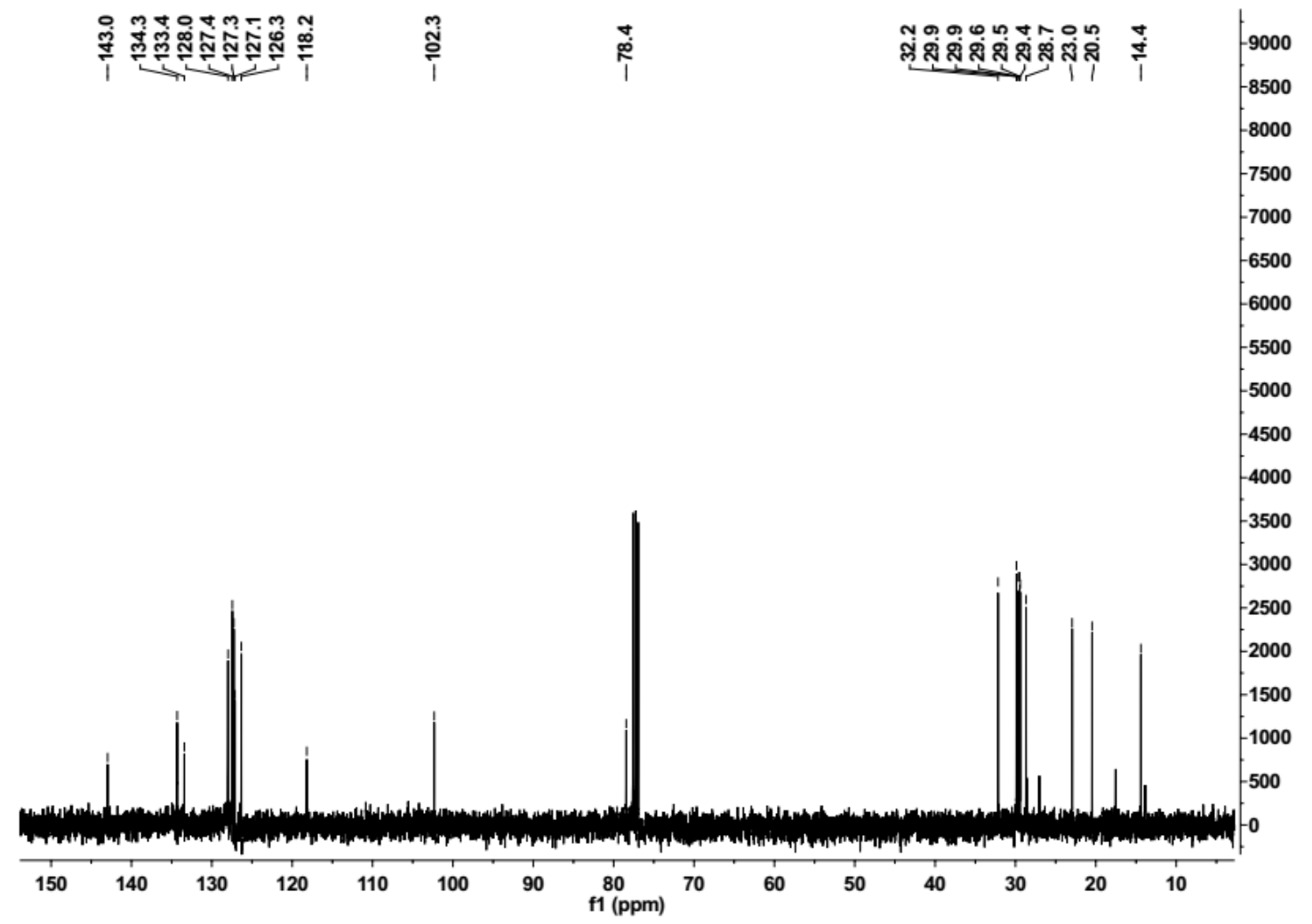


Figure S25. ${ }^{1} \mathrm{H}$ NMR spectrum of $\mathbf{3 e}$ in $\mathrm{CDCl}_{3}$.

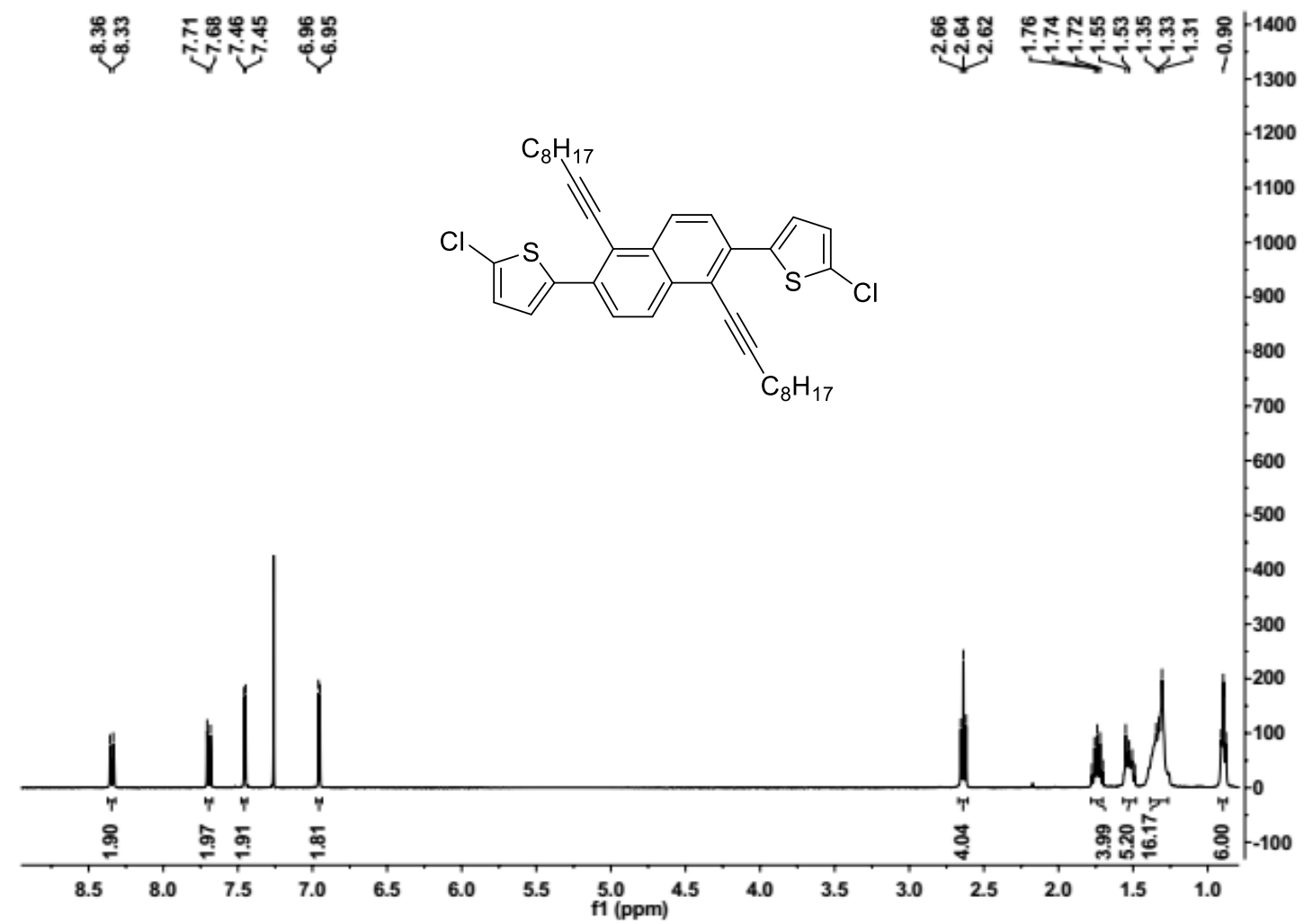

Figure S26. ${ }^{13} \mathrm{C}$ NMR spectrum of $\mathbf{3 e}$ in $\mathrm{CDCl}_{3}$.

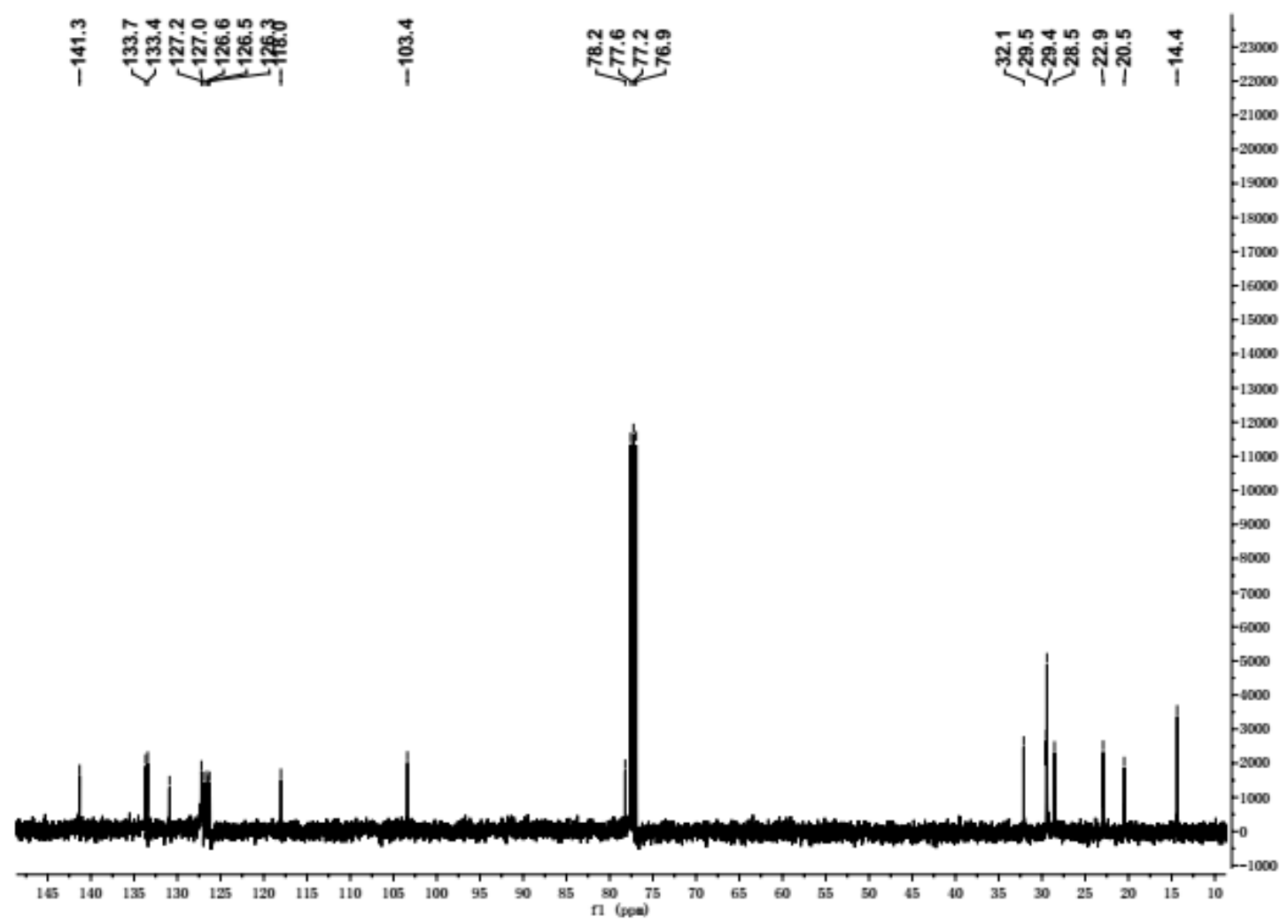


Figure S27. ${ }^{1} \mathrm{H} \mathrm{NMR}$ spectrum of $\mathbf{3 f}$ in $\mathrm{CDCl}_{3}$.

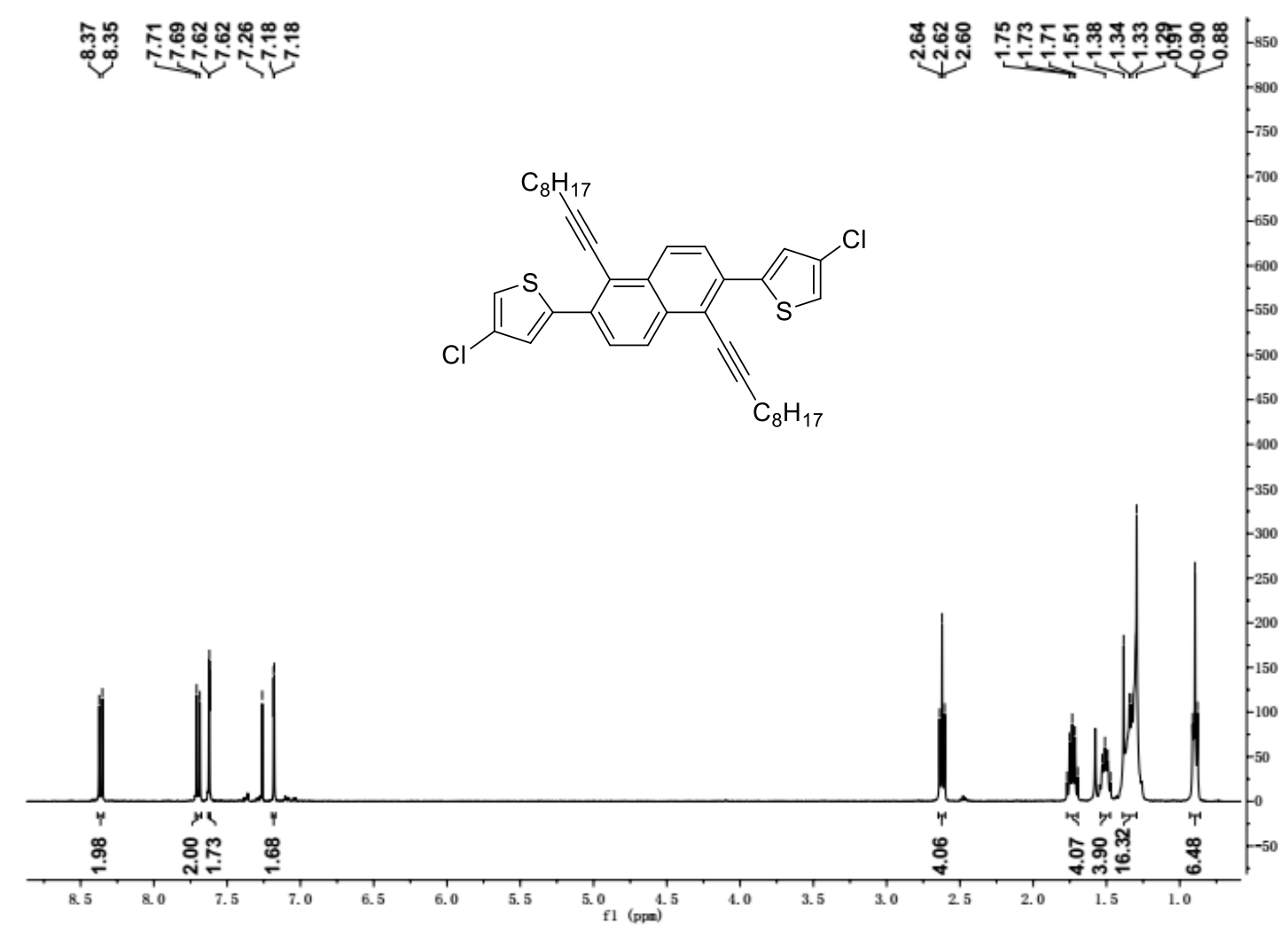

Figure S28. ${ }^{13} \mathrm{C}$ NMR spectrum of $\mathbf{3 f}$ in $\mathrm{CDCl}_{3}$.

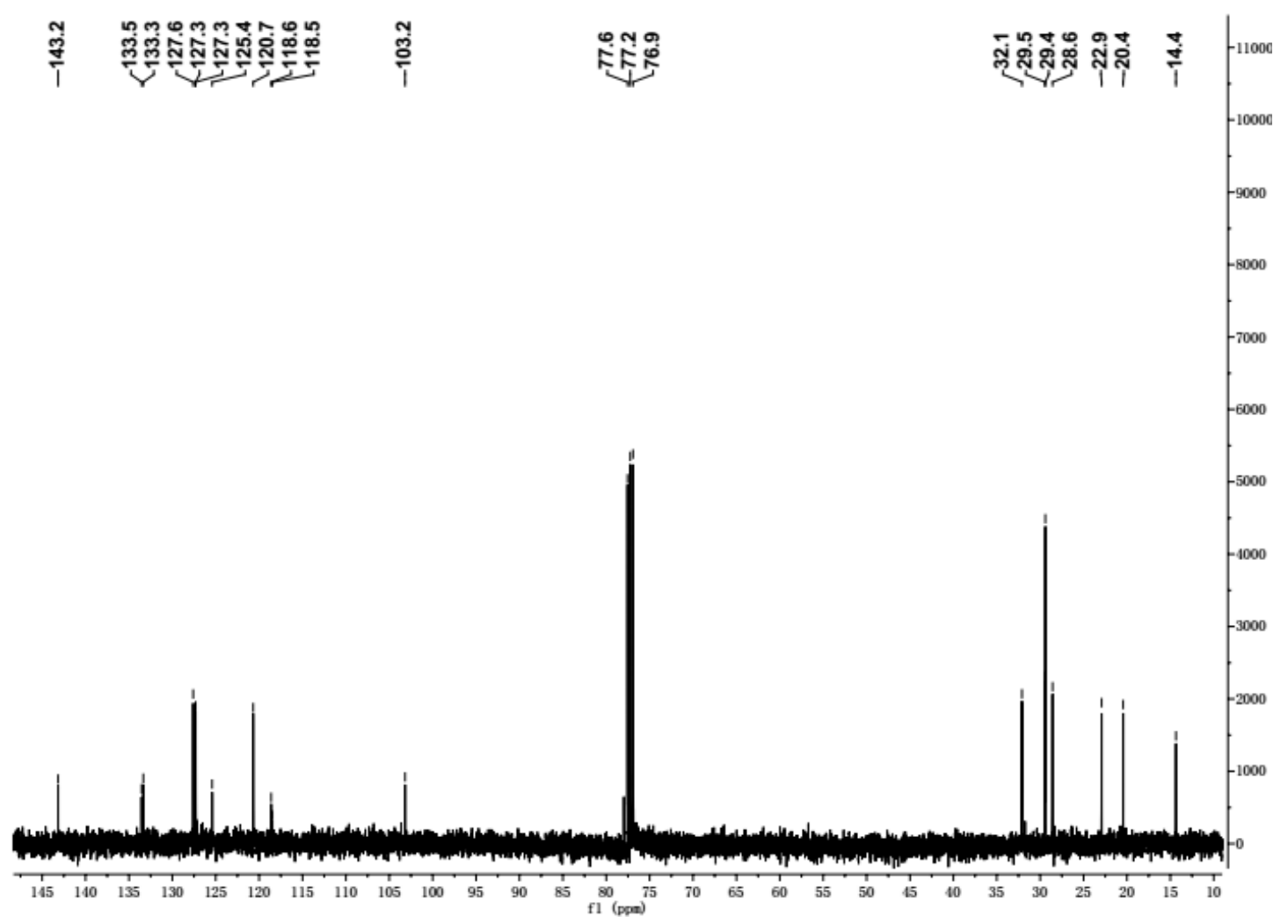


Figure S29. ${ }^{1} \mathrm{H}$ NMR spectrum of $\mathbf{3 g}$ in $\mathrm{CDCl}_{3}$.
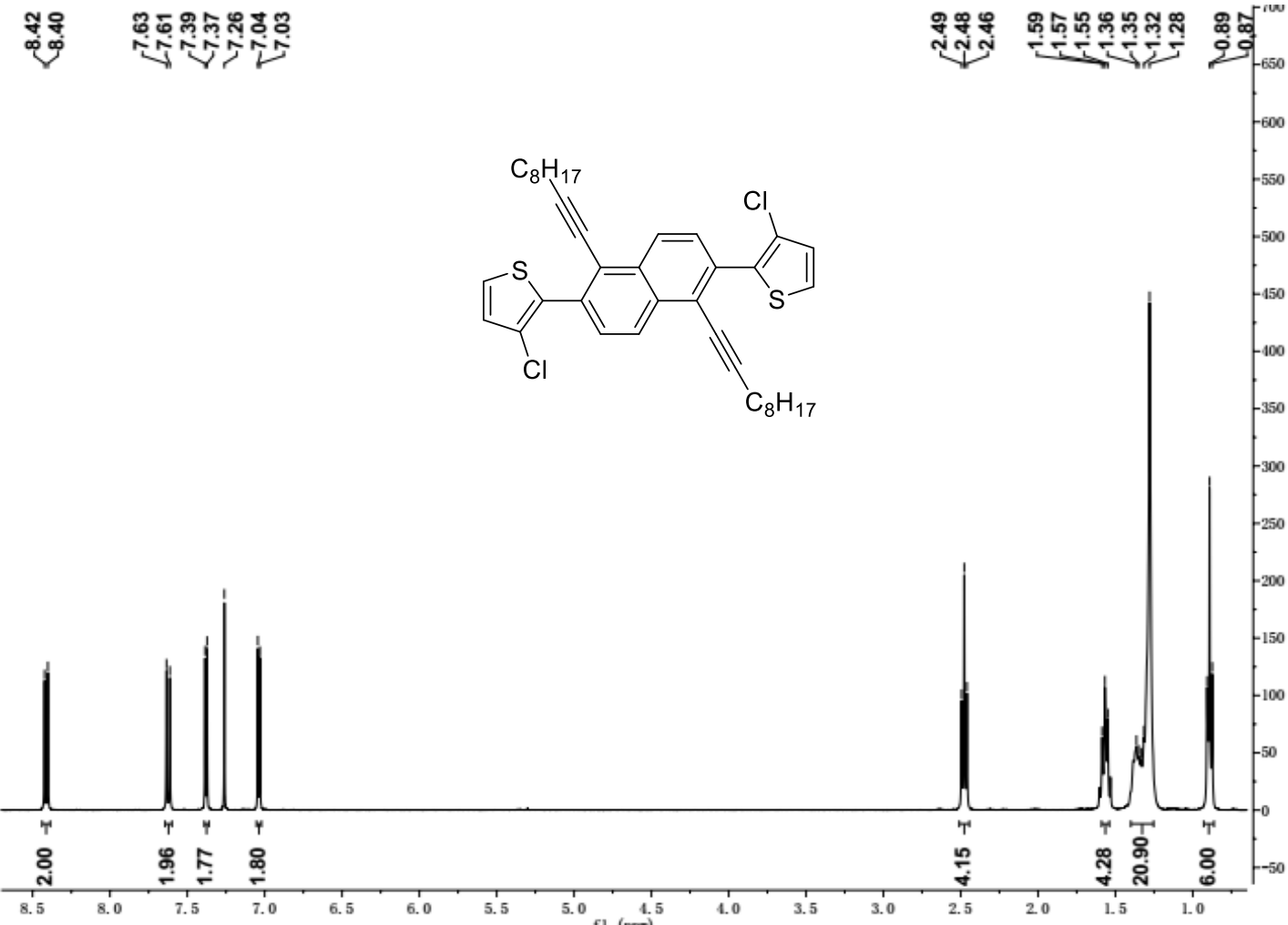

Figure S30. ${ }^{13} \mathrm{C}$ NMR spectrum of $\mathbf{3 g}$ in $\mathrm{CDCl}_{3}$.

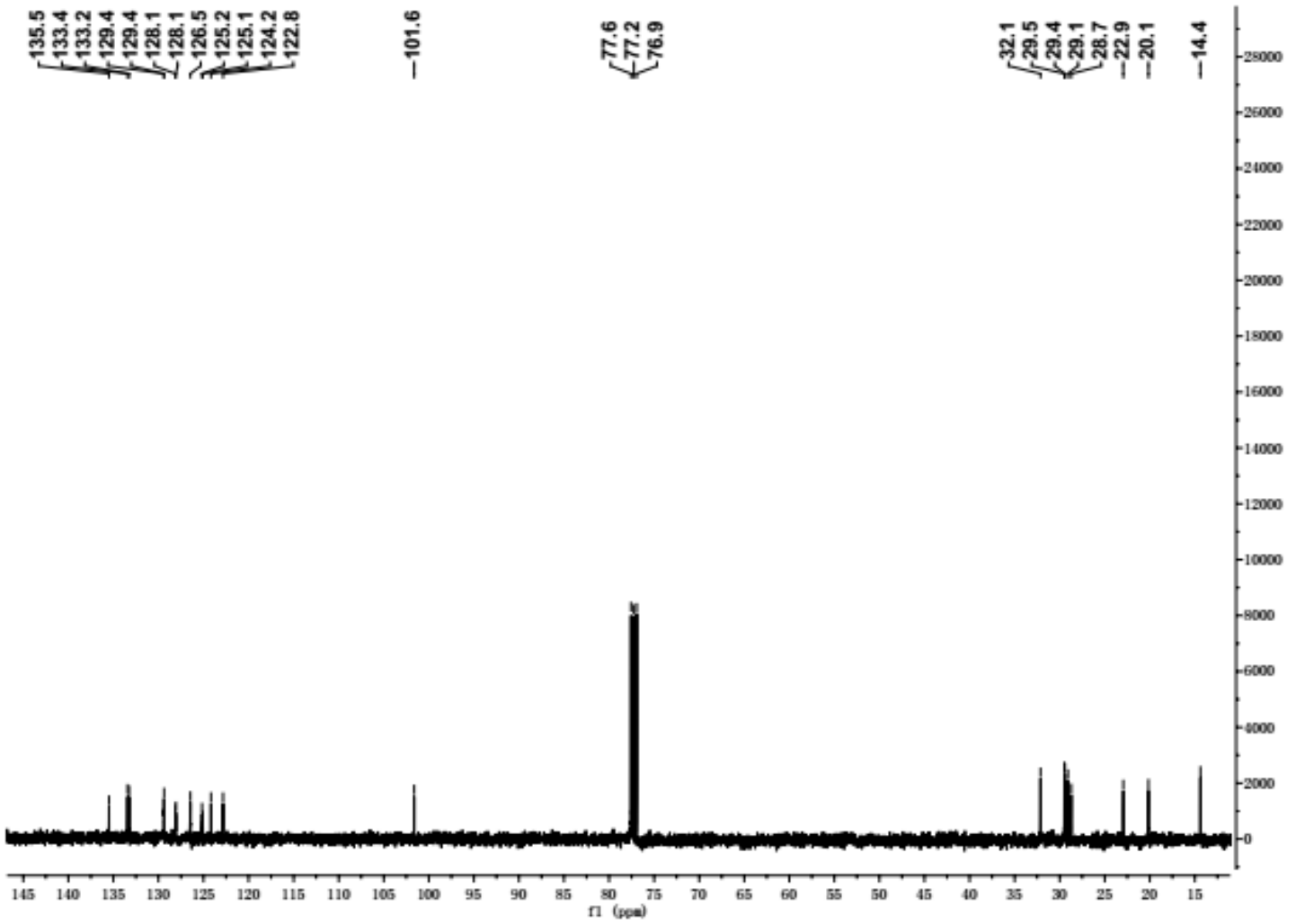


Figure S31. ${ }^{1} \mathrm{H}$ NMR spectrum of $\mathbf{C}_{4}-\mathbf{S S}$ in $\mathrm{CD}_{2} \mathrm{Cl}_{2}$.

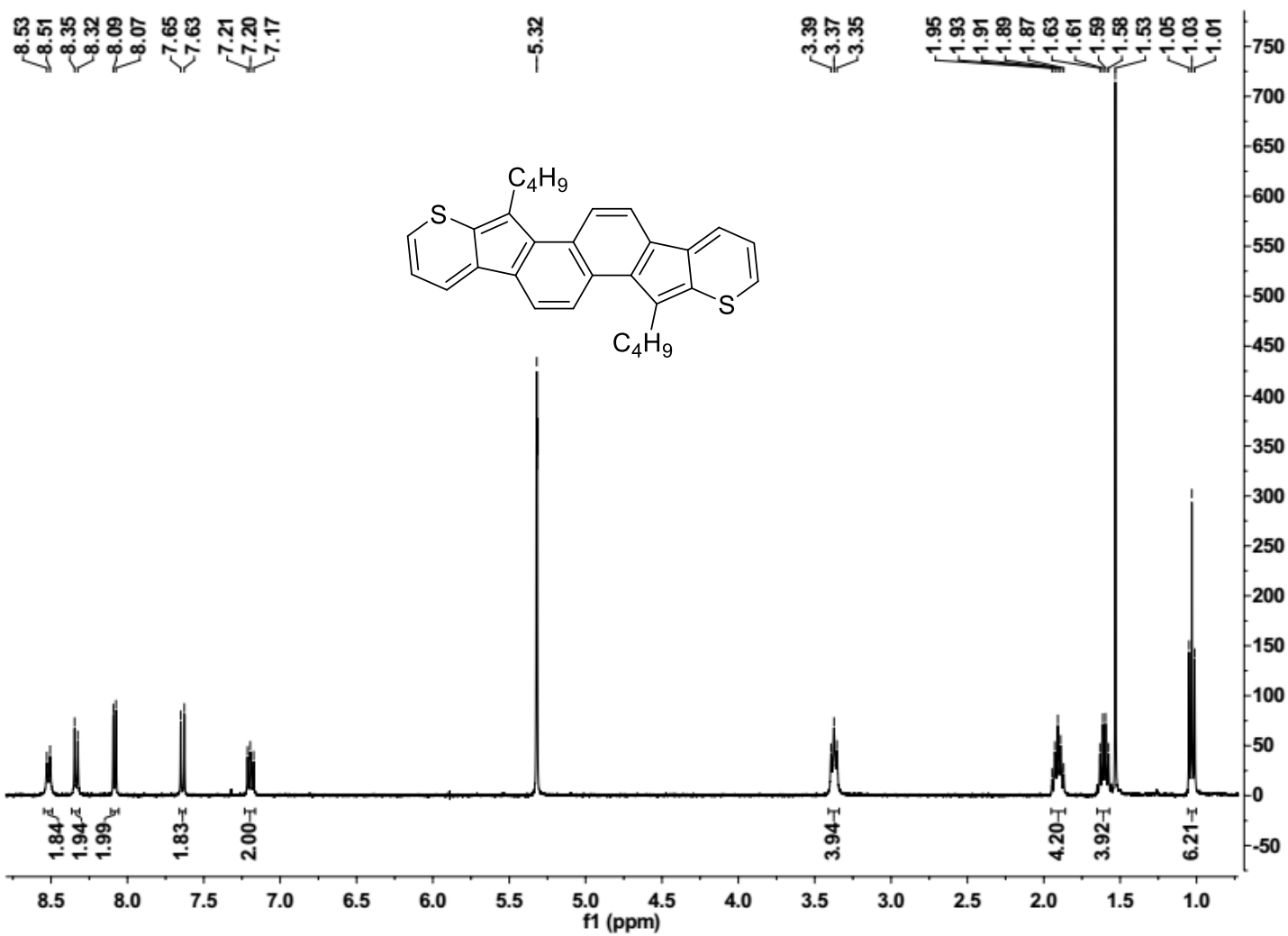

Figure S32. ${ }^{13} \mathrm{C}$ NMR spectrum of $\mathbf{C}_{4}-\mathrm{SS}$ in $\mathrm{CD}_{2} \mathrm{Cl}_{2}$.

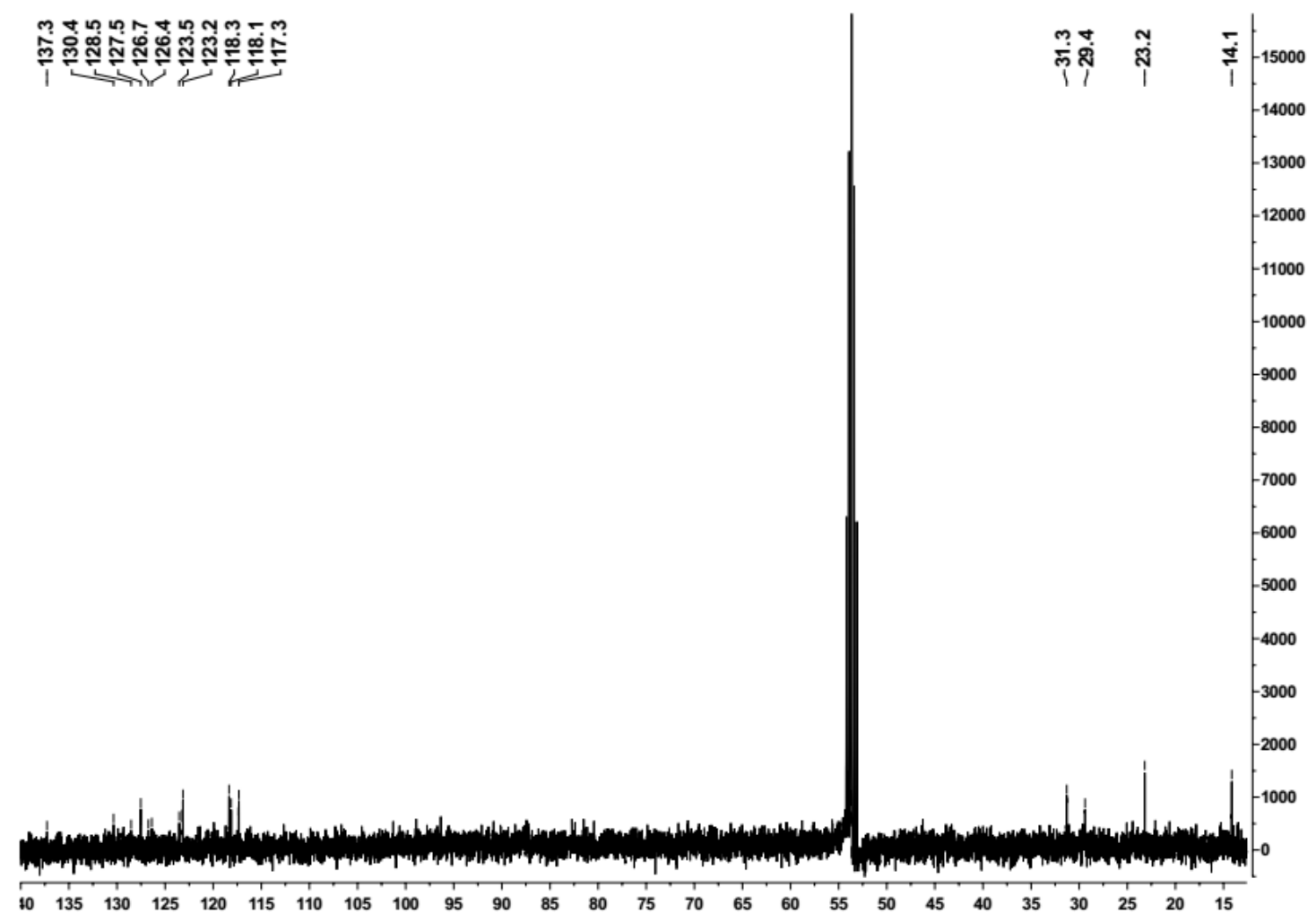


Figure S33. ${ }^{1} \mathrm{H}$ NMR spectrum of $\mathbf{C}_{6}-\mathbf{S S}$ in $\mathrm{CD}_{2} \mathrm{Cl}_{2}$.

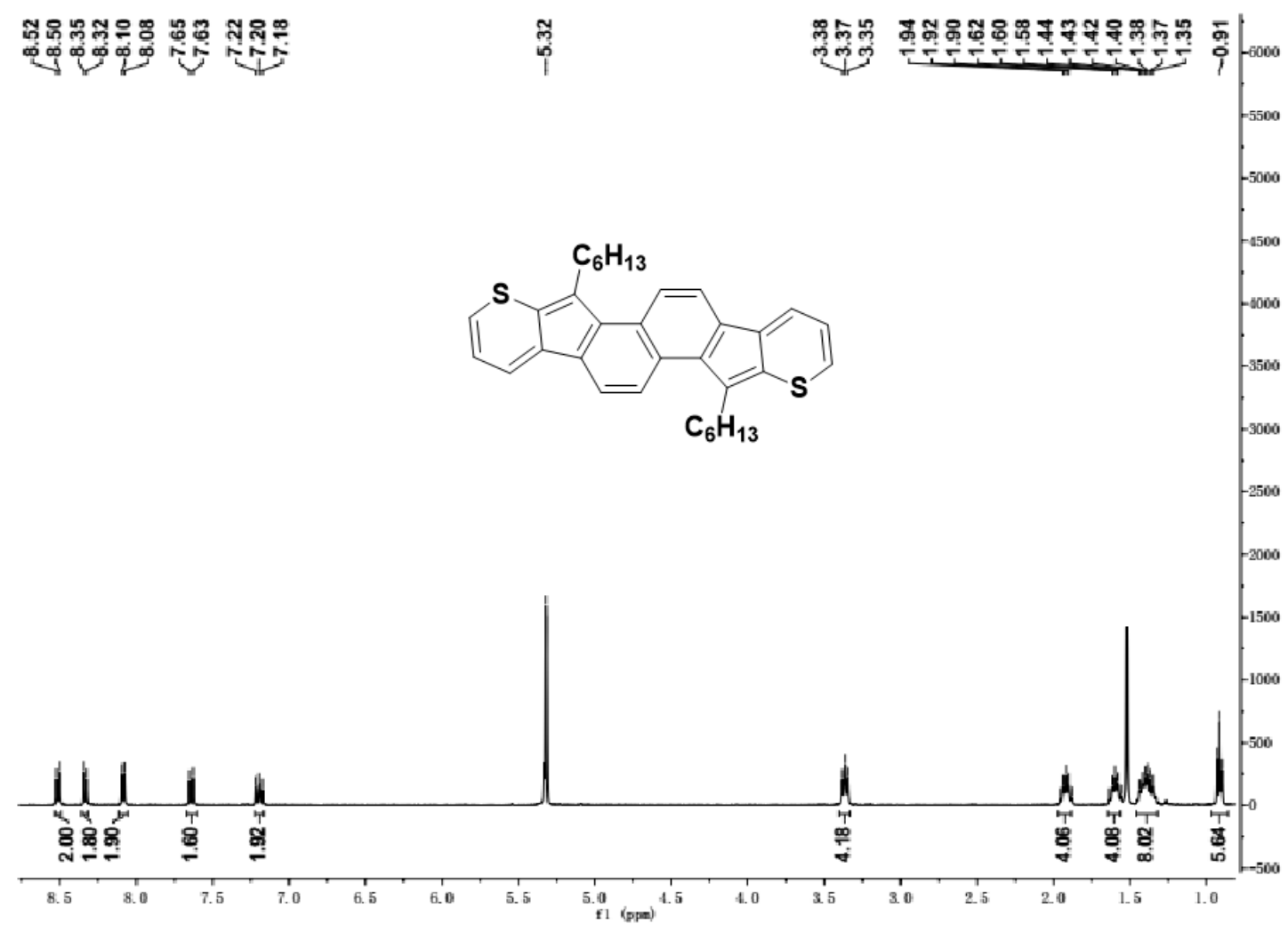

Figure S34. ${ }^{1} \mathrm{H}$ NMR spectrum of $\mathbf{C} 8-S S$ in $\mathrm{CD}_{2} \mathrm{Cl}_{2}$.

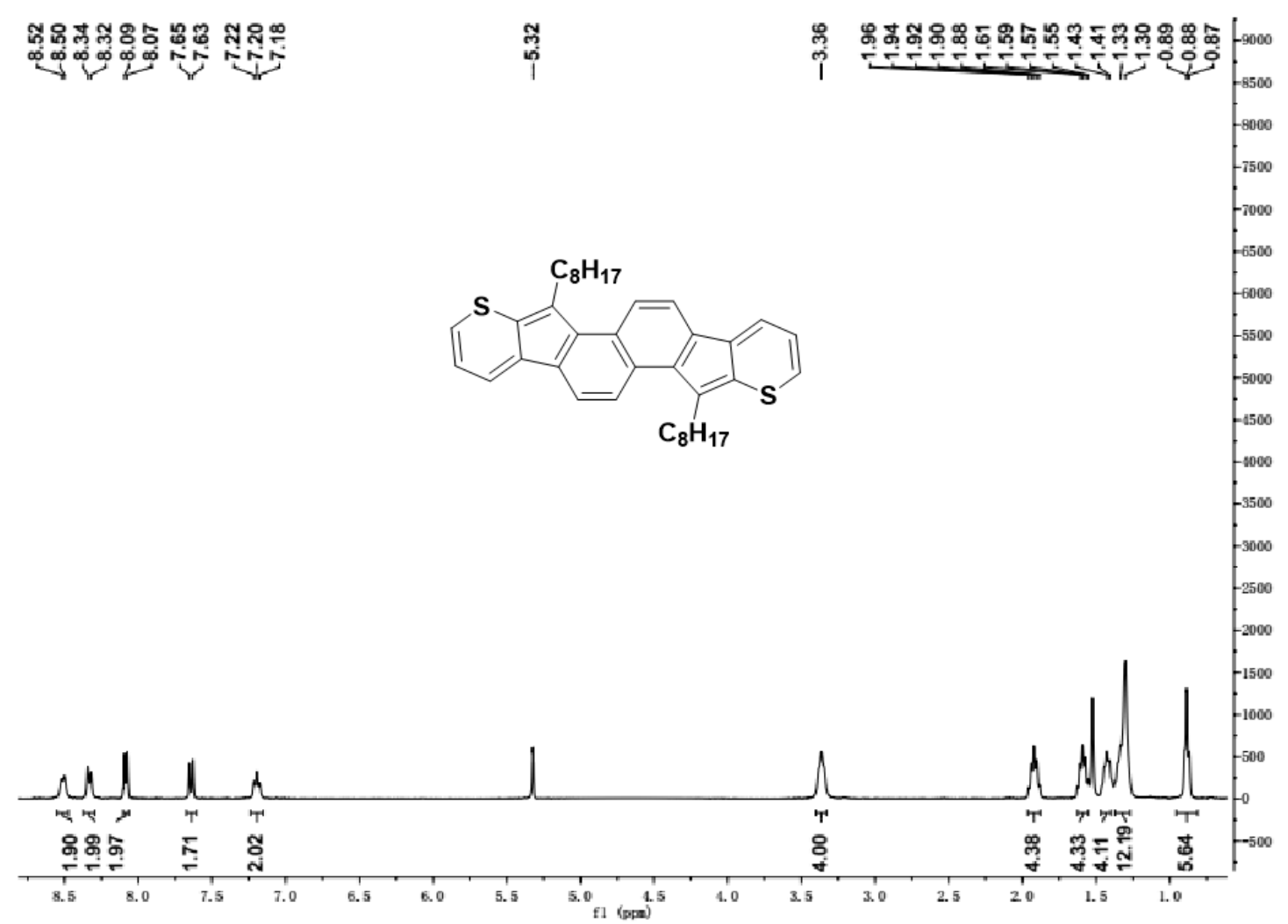


Figure S35. ${ }^{13} \mathrm{C}$ NMR spectrum of $\mathbf{C}_{8}-\mathbf{S S}$ in $\mathrm{CDCl}_{3}$.

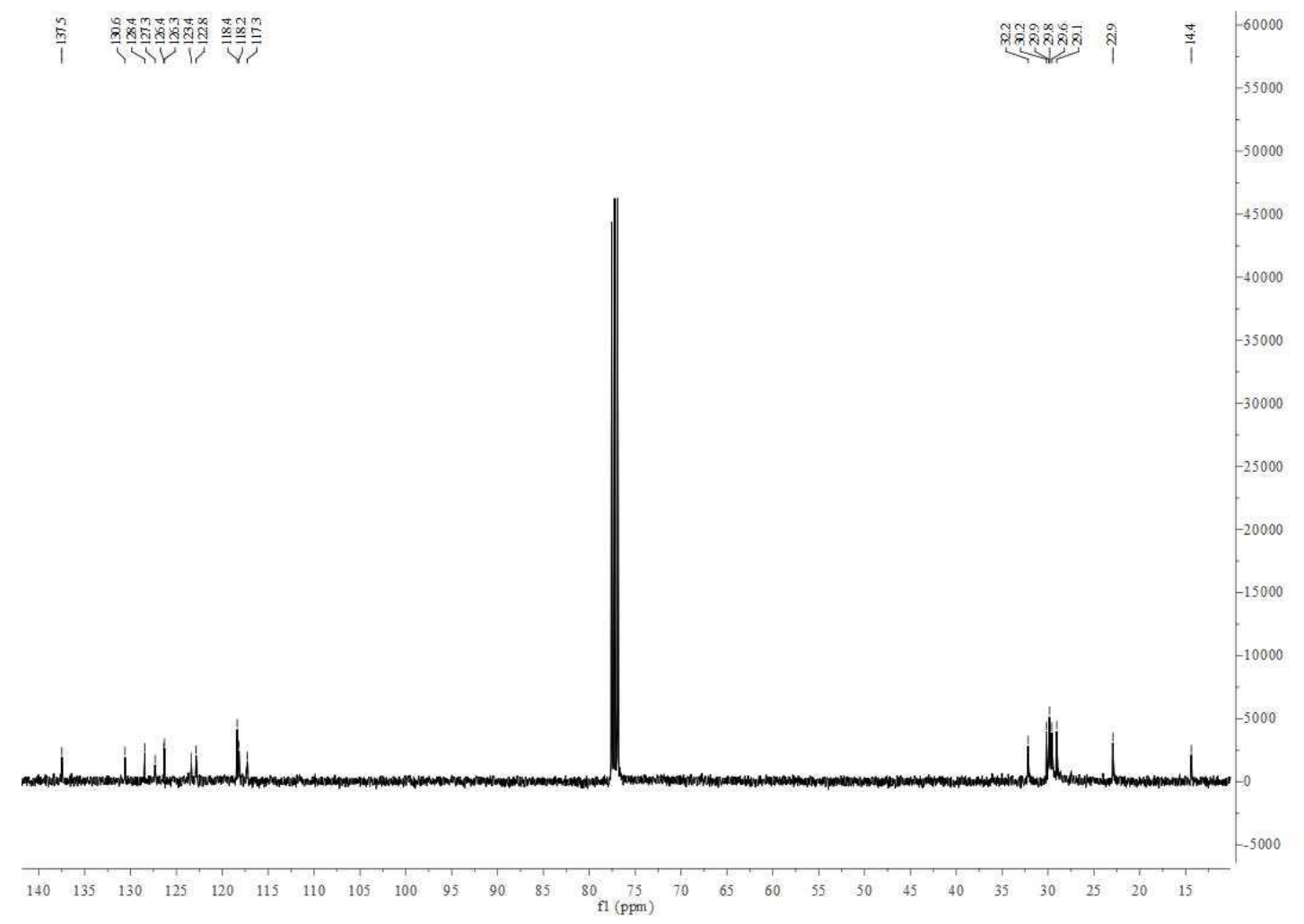

Figure S36. ${ }^{1} \mathrm{H}$ NMR spectrum of $\mathbf{C}_{\mathbf{1 0}}-\mathbf{S S}$ in $\mathrm{CDCl}_{3}$.

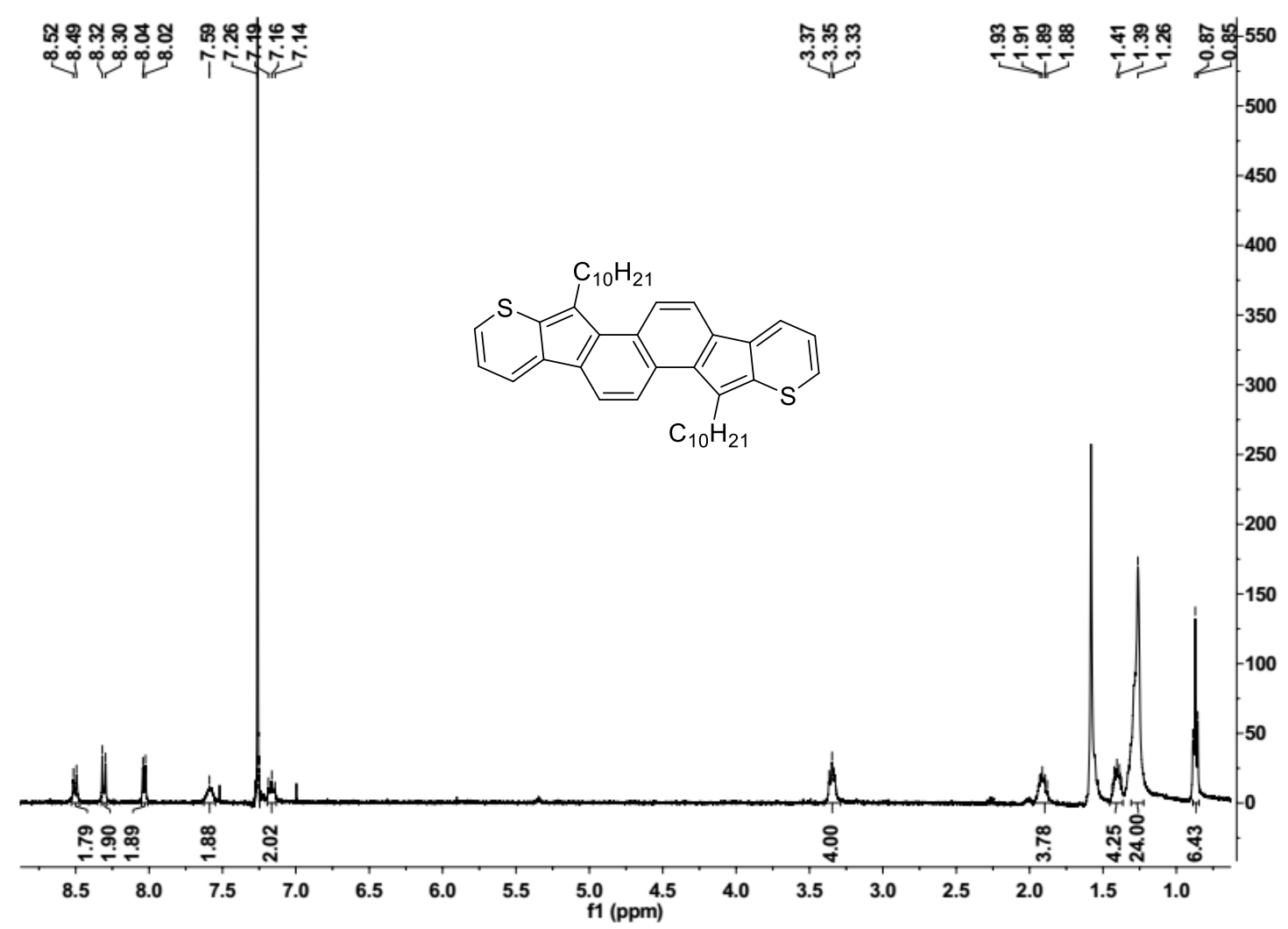


Figure S37. ${ }^{13} \mathrm{C}$ NMR spectrum of $\mathrm{C}_{10}-\mathrm{SS}$ in $\mathrm{C}_{2} \mathrm{D}_{2} \mathrm{Cl}_{4}$.

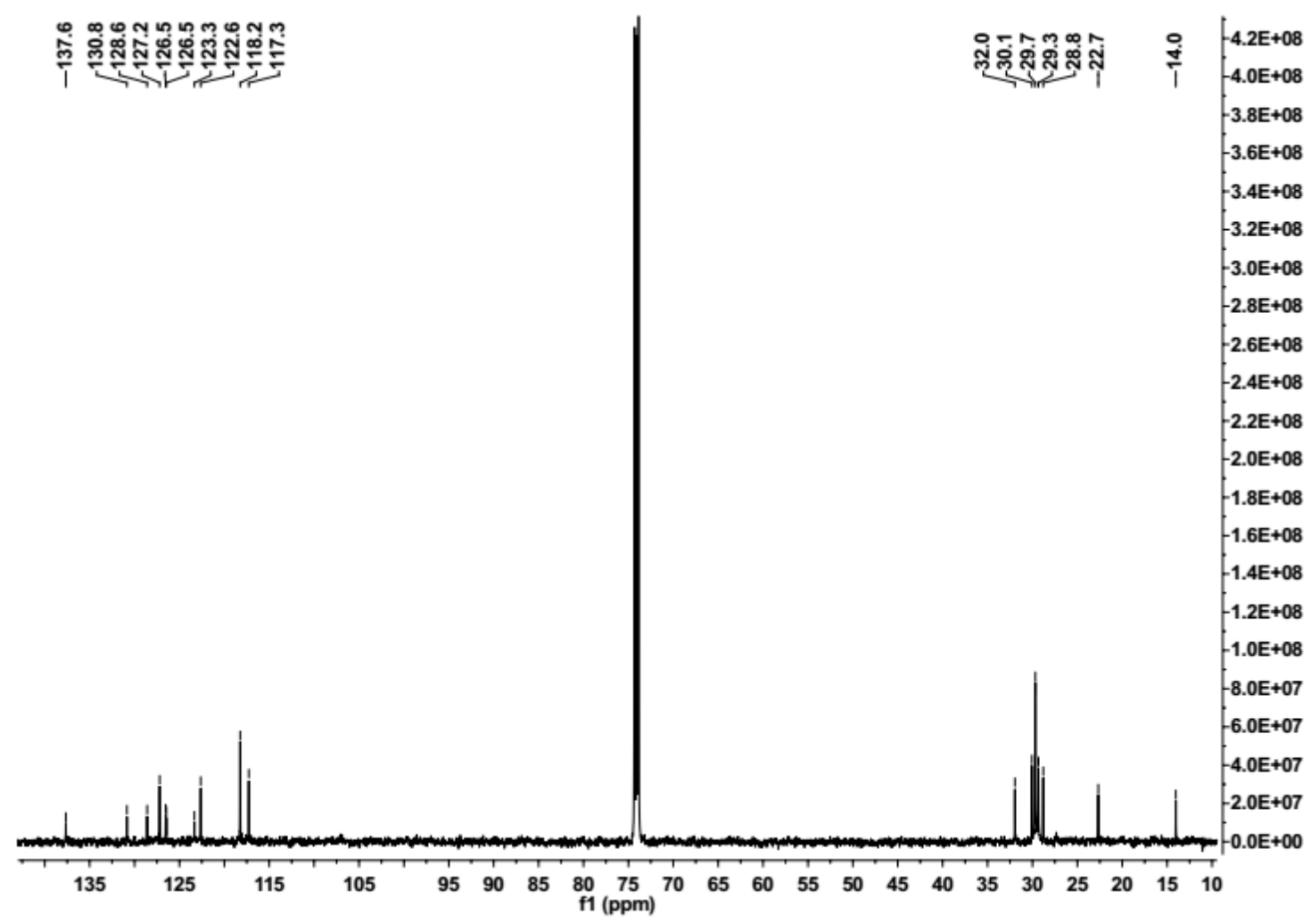

Figure S38. ${ }^{1} \mathrm{H}$ NMR spectrum of $\mathbf{C} \mathbf{8}-\mathbf{S S}-\mathbf{C l}^{\mathbf{2}}$ in $\mathrm{CD}_{2} \mathrm{Cl}_{2}$.

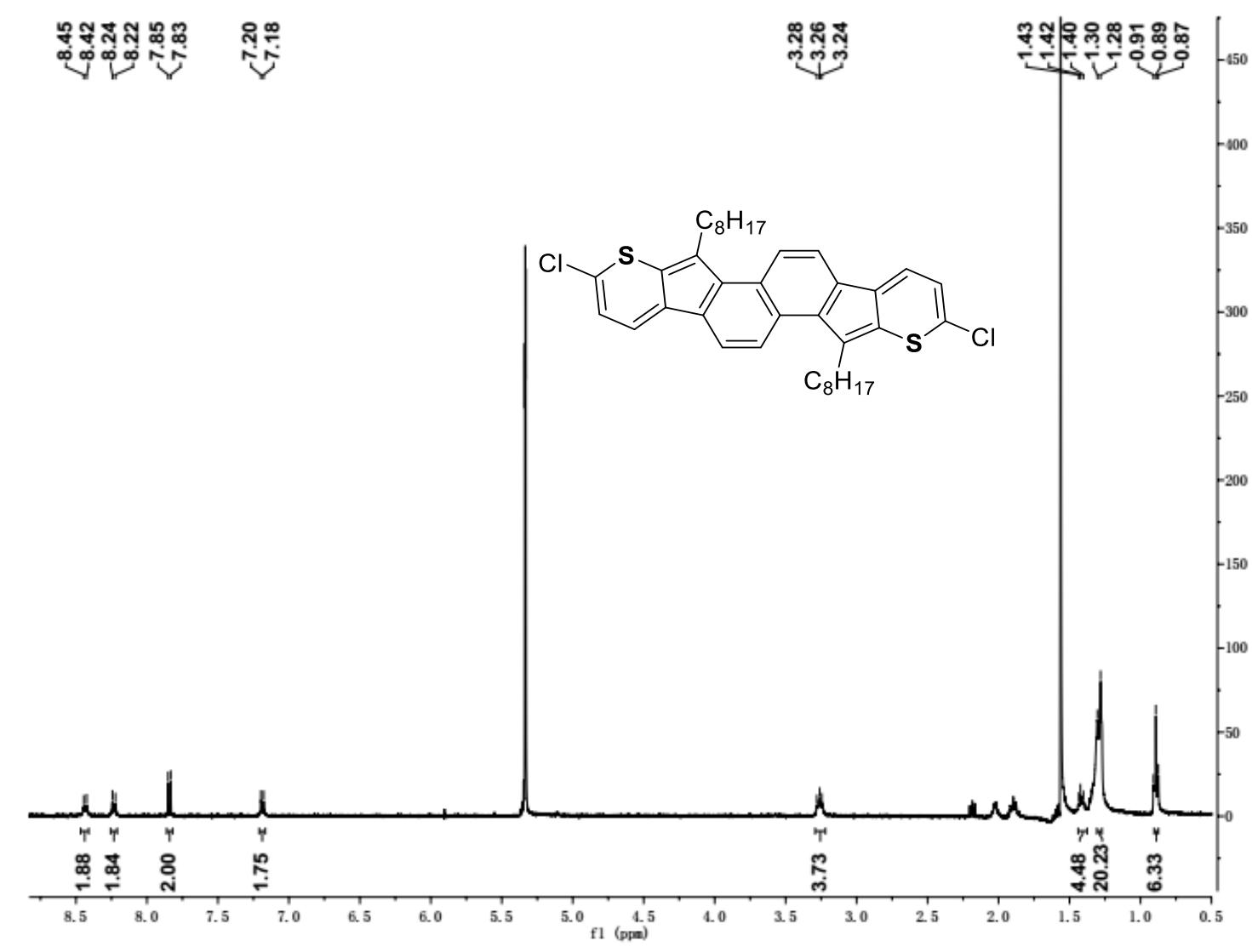


Figure S39. ${ }^{1} \mathrm{H}$ NMR spectrum of $\mathbf{C}_{8}-\mathbf{S S}-\mathbf{C l}^{3}$ in $\mathrm{CDCl}_{3}$.

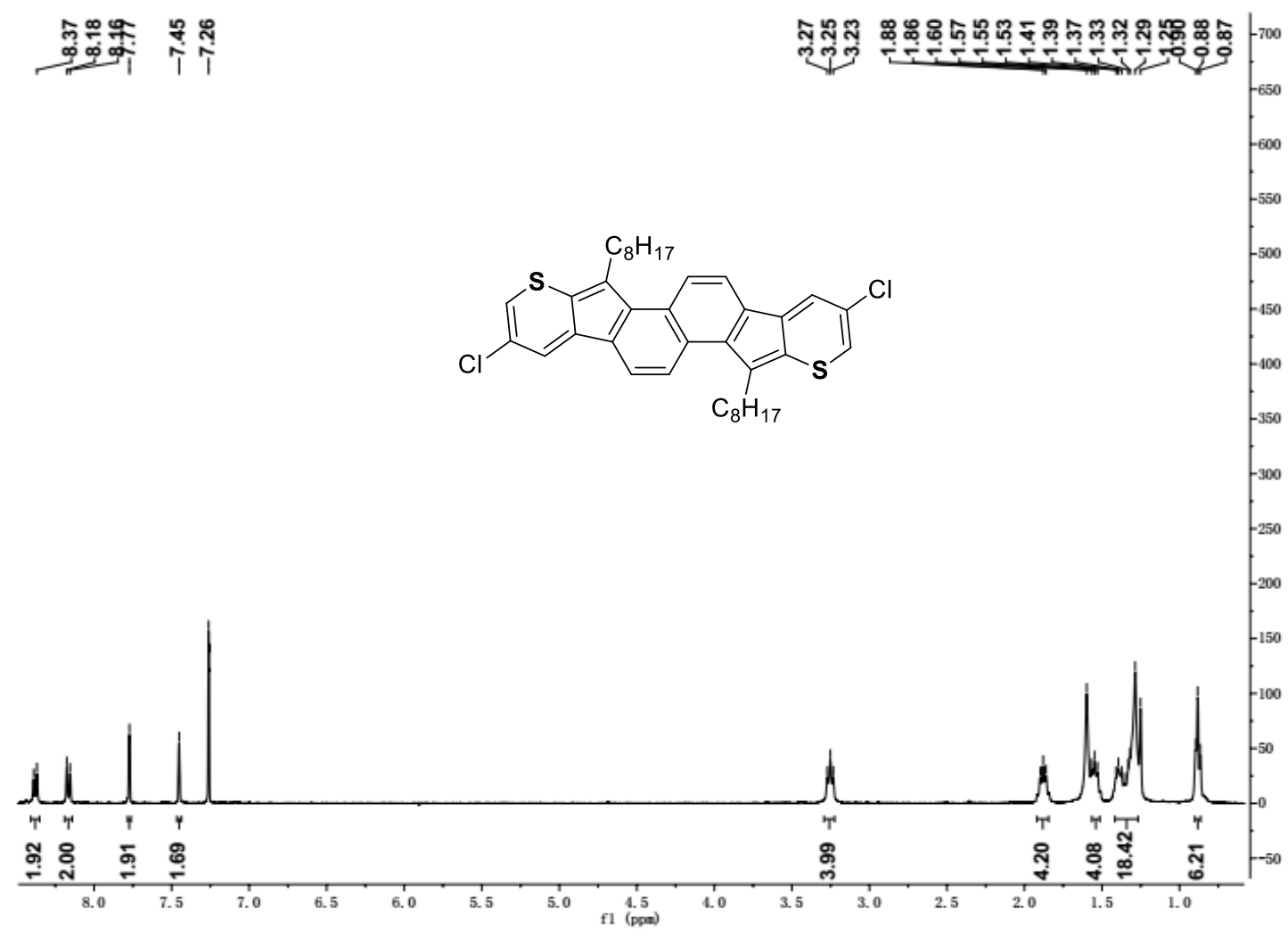

Figure S40. ${ }^{13} \mathrm{C}$ NMR spectrum of $\mathbf{C r}_{\mathbf{8}}-\mathbf{S S}-\mathbf{C l}^{3}$ in $\mathrm{CDCl}_{3}$.

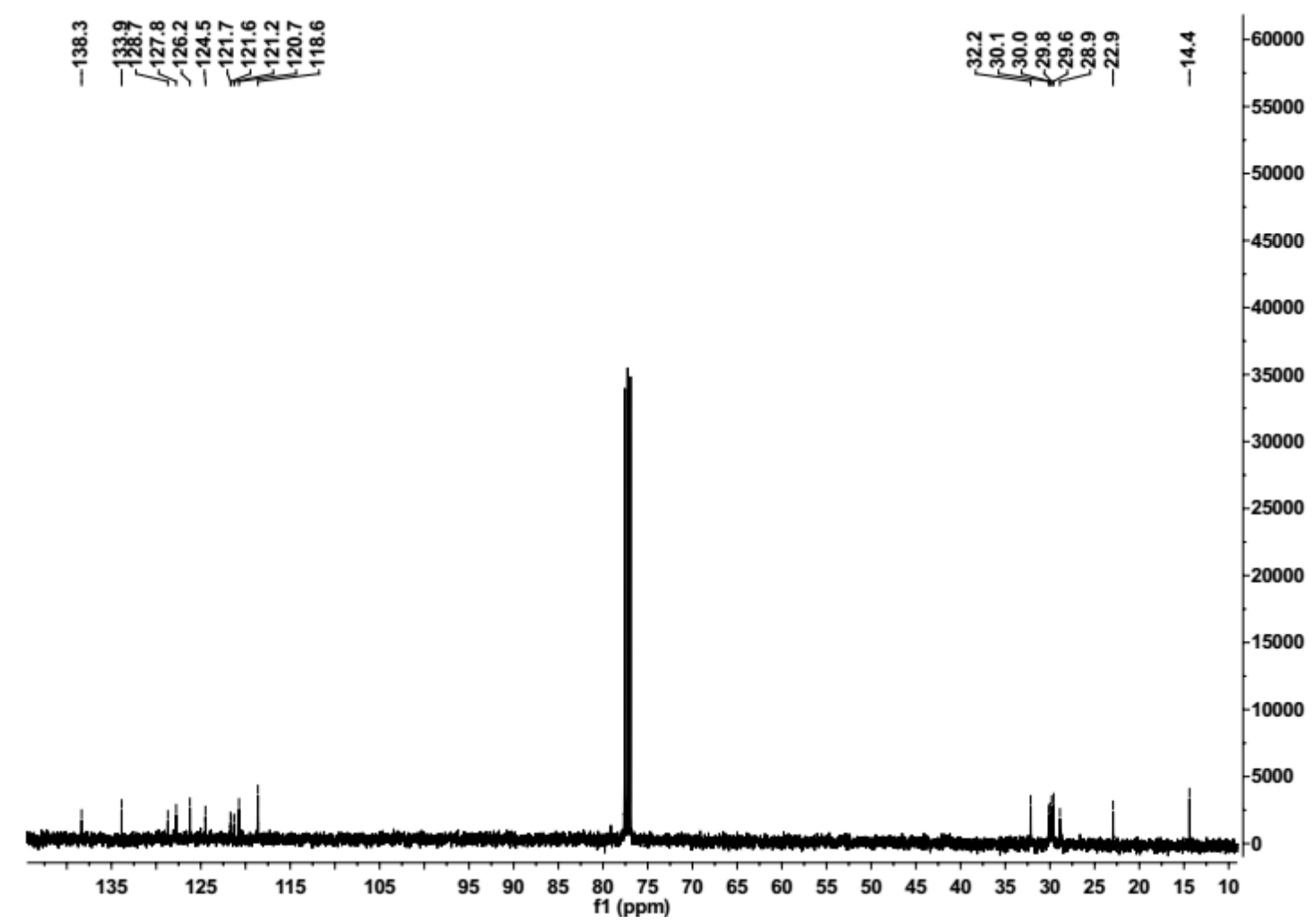


Figure S41. ${ }^{1} \mathrm{H}$ NMR spectrum of $\mathbf{C}_{8}-\mathbf{S S}-\mathbf{C l}^{4}$ in THF- $d_{8}$.

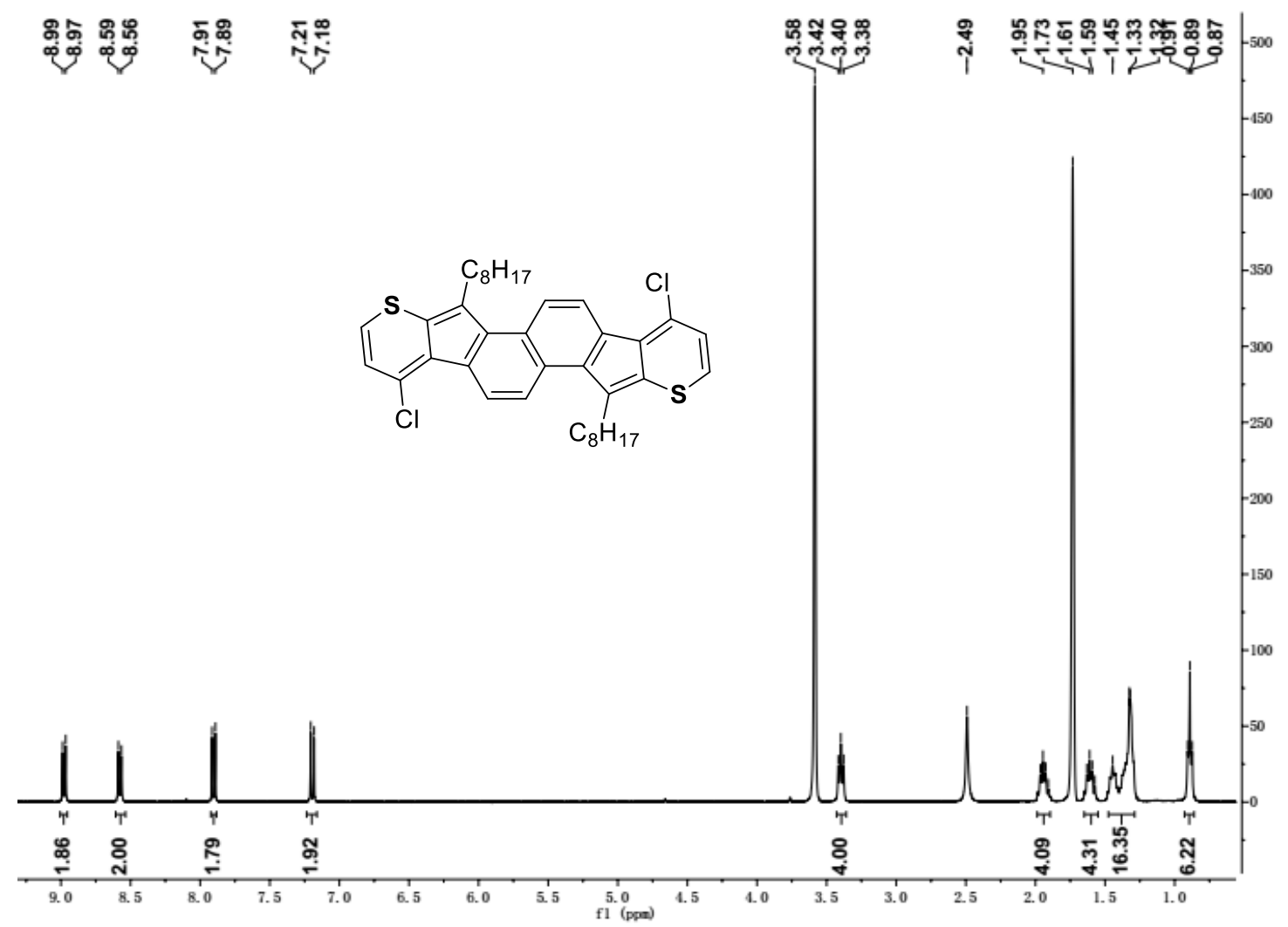




\section{References}

S1. Shinamura, S.; Osaka, I.; Miyazaki, E.; Nakao, A.; Yamagishi, M.; Takeya, J.; Takimiya, K. Linear- and Angular-Shaped Naphthodithiophenes: Selective Synthesis, Properties, and Application to Organic Field-Effect Transistors. J. Am. Chem. Soc. 2011, 133, 5024-5035.

S2. Karmel, C.; Chen, Z.; Hartwig, J. F.; Iridium-Catalyzed Silylation of C-H Bonds in Unactivated Arenes: A Sterically Encumbered Phenanthroline Ligand Accelerates Catalysis. $J$. Am. Chem. Soc. 2019, 141, 7063-7072.

S3. Gaussian 16, Revision C.01, Frisch, M. J.; Trucks, G. W.; Schlegel, H. B.; Scuseria, G. E.; Robb, M. A.; Cheeseman, J. R.; Scalmani, G.; Barone, V.; Petersson, G. A.; Nakatsuji, H.; Li, X.; Caricato, M.; Marenich, A. V.; Bloino, J.; Janesko, B. G.; Gomperts, R.; Mennucci, B.; Hratchian, H. P.; Ortiz, J. V.; Izmaylov, A. F.; Sonnenberg, J. L.; Williams-Young, D.; Ding, F.; Lipparini, F.; Egidi, F.; Goings, J.; Peng, B.; Petrone, A.; Henderson, T.; Ranasinghe, D.; Zakrzewski, V. G.; Gao, J.; Rega, N.; Zheng, G.; Liang, W.; Hada, M.; Ehara, M.; Toyota, K.; Fukuda, R. Hasegawa, J.; Ishida, M.; Nakajima, T.; Honda, Y.; Kitao, O.; Nakai, H.; Vreven, T.; Throssell, K.; Montgomery, Jr. J. A.; Peralta, J. E.; Ogliaro, F.; Bearpark, M. J.; Heyd, J. J.; Brothers, E. N.; Kudin, K. N.; Staroverov, V. N.; Keith, T. A.; Kobayashi, R.; Normand, J.; Raghavachari, K.; Rendell, A. P.; Burant, J. C.; Iyengar, S. S.; Tomasi, J.; Cossi, M.; Millam, J. M.; Klene, M.; Adamo, C.; Cammi, R.; Ochterski, J. W.; Martin, R. L.; Morokuma, K.; Farkas, O.; Foresman, J. B.; Fox, D. J. Gaussian, Inc., Wallingford CT, 2019.

S4. Huang, J. S.; Kertesz, M. Intermolecular Transfer Integrals for Organic Molecular Materials: Can Basis Set Convergence Be Achieved? Chem. Phys. Lett. 2004, 390, 110-115.

S5. Yang, X. D.; Wang, L. J.; Wang, C. L.; Long, W.; Shuai, Z. G. Influences of Crystal Structures and Molecular Sizes on the Charge Mobility of Organic Semiconductors: Oligothiophenes. Chem. Mater. 2008, 20, 3205-3211. 